\title{
SUPPLEMENTAL INFORMATION Activation of Cellulose with Alkaline Earth Metals
}

\author{
Gregory G. Facas ${ }^{1}$, Vineet Maliekkal ${ }^{1}$, Matthew Neurock ${ }^{1}$, Paul J. Dauenhauer ${ }^{1 *}$ \\ ${ }^{1}$ University of Minnesota, Department of Chemical Engineering and Materials Science, 421 Washington Ave. SE, \\ Minneapolis, MN, 55455. \\ *Corresponding Author: mneurock@umn.edu \& hauer@umn.edu
}

\section{TABLE OF CONTENTS}

Supplemental Note 1. Tabulated $\alpha$-Cyclodextrin Conversion Data .........................Page S2

Supplemental Note 2. Additional transition state for dual Mg catalyzed mechanism........Page S8

Supplemental Note 3. Coordinates for DFT structures.................................Page S9 


\section{Supplemental Note 2. Tabulated $\alpha$-Cyclodextrin conversion Data}

The following tables contain the time resolved conversion data for catalyzed $\alpha$-cyclodextrin activation presented in the main paper. Tables S1-A to S1-L show the progression of $\alpha$-cyclodextrin conversion with different pulse lengths (20-2000 ms) for a range of temperatures $\left(370-430{ }^{\circ} \mathrm{C}\right)$ and a range of $\mathrm{Mg}\left(\mathrm{NO}_{3}\right)_{2}$ concentrations (0.1-0.5 mmol Ca(NO$\left.)_{2} / \mathrm{g} \alpha-\mathrm{CD}\right)$. The error columns represent a $95 \%$ confidence interval based on triplicate of experiments for each experimental condition.

Table S1-A. Conversion of cyclodextrin with $\mathrm{Mg}^{2+}$ catalyst at $370{ }^{\circ} \mathrm{C}$

\begin{tabular}{|c|c|c|c|c|c|c|}
\hline Temperature $\left[{ }^{\circ} \mathbf{C}\right]$ & \multicolumn{6}{|c|}{370} \\
\hline $\begin{array}{c}\text { Magnesium Concentration } \\
{\left[\mathrm{mmol} \mathrm{Mg}^{2+} / \mathrm{g}-\mathrm{CD}\right]}\end{array}$ & \multicolumn{2}{|c|}{ Pure CD } & \multicolumn{2}{|c|}{0.1} & \multicolumn{2}{|l|}{0.2} \\
\hline Pulse Length [ms] & $\begin{array}{c}\text { Conversion } \\
{[\%]}\end{array}$ & Error [\%] & $\begin{array}{c}\text { Conversion } \\
{[\%]}\end{array}$ & Error [\%] & Conversion [\%] & Error $[\%]$ \\
\hline 20 & 1.16 & 1.2 & 1.72 & 0.8 & 0.91 & 1.2 \\
\hline 50 & 1.92 & 1.8 & 2.68 & 1.0 & 2.79 & 0.9 \\
\hline 75 & 2.37 & 0.1 & 3.29 & 1.6 & 4.37 & 2.8 \\
\hline 100 & 4.32 & 2.0 & 4.40 & 1.3 & 5.91 & 2.1 \\
\hline 150 & 7.25 & 2.7 & 8.90 & 2.1 & 9.03 & 2.8 \\
\hline 200 & 8.16 & 0.8 & 12.91 & 1.4 & 12.64 & 1.3 \\
\hline 500 & 17.39 & 4.9 & 24.58 & 3.2 & 24.09 & 3.7 \\
\hline 1000 & 31.19 & 2.3 & 36.77 & 3.8 & 45.81 & 3.8 \\
\hline 2000 & 50.83 & 3.0 & 53.55 & 4.3 & 63.06 & 2.8 \\
\hline
\end{tabular}

Table S1-B. Conversion of cyclodextrin with $\mathrm{Mg}^{2+}$ catalyst at $370^{\circ} \mathrm{C}$

\begin{tabular}{|c|c|c|c|c|c|c|}
\hline Temperature [ ${ }^{\circ}$ C] & \multicolumn{2}{|c|}{370} \\
\hline $\begin{array}{c}\text { Magnesium Concentration } \\
\text { [mmol Mg }{ }^{2+} / \mathrm{g} \text {-CD }\end{array}$ & \multicolumn{2}{|c|}{0.3} & \multicolumn{2}{c|}{0.4} & \multicolumn{2}{c|}{0.5} \\
\hline Pulse Length [ms] & $\begin{array}{c}\text { Conversion } \\
{[\%]}\end{array}$ & Error [\%] & $\begin{array}{c}\text { Conversion } \\
{[\%]}\end{array}$ & Error [\%] & Conversion [\%] & Error [\%] \\
\hline 20 & 1.04 & 1.2 & 1.91 & 2.2 & 2.49 & 1.8 \\
\hline 50 & 3.84 & 1.1 & 5.81 & 2.3 & 5.65 & 1.7 \\
\hline 75 & 5.12 & 0.9 & 8.86 & 1.7 & 10.86 & 2.5 \\
\hline 100 & 7.26 & 1.5 & 12.89 & 2.2 & 13.24 & 1.9 \\
\hline 150 & 12.26 & 2.0 & 16.37 & 2.8 & 19.29 & 2.5 \\
\hline 200 & 23.27 & 4.0 & 24.37 & 2.7 & 28.00 & 3.0 \\
\hline 500 & 32.92 & 4.4 & 49.08 & 1.7 & 54.44 & 2.1 \\
\hline 1000 & 49.50 & 7.9 & 63.00 & 2.5 & 77.68 & 2.5 \\
\hline 2000 & 71.86 & 6.6 & 83.38 & 2.7 & 93.9 & 2.4 \\
\hline
\end{tabular}

Facas et al. 
Table S1-C. Conversion of cyclodextrin with $\mathrm{Mg}^{2+}$ catalyst at $385{ }^{\circ} \mathrm{C}$

\begin{tabular}{|c|c|c|c|c|c|c|}
\hline Temperature $\left[{ }^{\circ} \mathbf{C}\right]$ & \multicolumn{6}{|c|}{385} \\
\hline 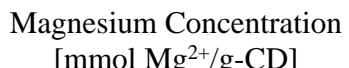 & \multicolumn{2}{|c|}{ Pure CD } & \multicolumn{2}{|c|}{0.1} & \multicolumn{2}{|l|}{0.2} \\
\hline Pulse Length [ms] & $\begin{array}{c}\text { Conversion } \\
{[\%]}\end{array}$ & Error $[\%]$ & $\begin{array}{c}\text { Conversion } \\
{[\%]}\end{array}$ & Error [\%] & Conversion [\%] & Error $[\%]$ \\
\hline 20 & 1.38 & 1.91 & 1.58 & 0.8 & 1.46 & 0.9 \\
\hline 50 & 4.08 & 2.19 & 2.11 & 1.0 & 3.73 & 0.5 \\
\hline 75 & 3.81 & 3.56 & 5.05 & 1.6 & 6.79 & 1.1 \\
\hline 100 & 4.22 & 3.31 & 5.71 & 1.3 & 10.81 & 2.0 \\
\hline 150 & 6.44 & 3.56 & 8.39 & 2.1 & 13.96 & 2.5 \\
\hline 200 & 10.12 & 2.73 & 11.63 & 1.4 & 18.56 & 1.9 \\
\hline 500 & 20.23 & 5.97 & 23.59 & 3.2 & 37.91 & 1.2 \\
\hline 1000 & 36.35 & 10.11 & 46.61 & 3.8 & 58.03 & 3.6 \\
\hline 2000 & 55.41 & 7.20 & 63.00 & 4.3 & 85.07 & 2.2 \\
\hline
\end{tabular}

Table S1-D. Conversion of cyclodextrin with $\mathrm{Mg}^{2+}$ catalyst at $385{ }^{\circ} \mathrm{C}$

\begin{tabular}{|c|c|c|c|c|c|c|}
\hline Temperature $\left[{ }^{\circ} \mathbf{C}\right]$ & \multicolumn{6}{|c|}{385} \\
\hline $\begin{array}{c}\text { Magnesium Concentration } \\
{\left[\mathrm{mmol}^{2+} / \mathrm{g}-\mathrm{CD}\right]}\end{array}$ & \multicolumn{2}{|c|}{0.3} & \multicolumn{2}{|c|}{0.4} & \multicolumn{2}{|l|}{0.5} \\
\hline Pulse Length [ms] & $\begin{array}{c}\text { Conversion } \\
{[\%]} \\
\end{array}$ & Error $[\%]$ & $\begin{array}{c}\text { Conversion } \\
{[\%]} \\
\end{array}$ & Error $[\%]$ & Conversion [\%] & Error [\%] \\
\hline 20 & 2.79 & 1.7 & 1.86 & 0.5 & 6.06 & 1.8 \\
\hline 50 & 7.37 & 1.1 & 8.81 & 3.4 & 13.50 & 1.7 \\
\hline 75 & 12.72 & 3.3 & 16.49 & 1.1 & 22.91 & 2.5 \\
\hline 100 & 16.45 & 1.3 & 21.56 & 1.0 & 30.19 & 1.9 \\
\hline 150 & 21.19 & 0.9 & 28.54 & 1.4 & 39.22 & 2.5 \\
\hline 200 & 25.69 & 1.5 & 37.31 & 3.5 & 51.57 & 3.0 \\
\hline 500 & 52.21 & 4.4 & 65.44 & 4.9 & 76.08 & 2.1 \\
\hline 1000 & 77.01 & 1.63 & 88.80 & 4.2 & 89.00 & 2.5 \\
\hline 2000 & 90.19 & 5.6 & 93.90 & 3.3 & 88.39 & 2.4 \\
\hline
\end{tabular}


Table S1-E. Conversion of cyclodextrin with $\mathrm{Mg}^{2+}$ catalyst at $400{ }^{\circ} \mathrm{C}$

\begin{tabular}{|c|c|c|c|c|c|c|}
\hline Temperature $\left[{ }^{\circ} \mathbf{C}\right]$ & \multicolumn{6}{|c|}{400} \\
\hline Magnesium Concentration & \multicolumn{2}{|c|}{ Pure CD } & \multicolumn{2}{|c|}{0.1} & \multicolumn{2}{|l|}{0.2} \\
\hline Pulse Length [ms] & $\begin{array}{c}\text { Conversion } \\
{[\%]}\end{array}$ & Error [\%] & $\begin{array}{c}\text { Conversion } \\
{[\%]}\end{array}$ & Error $[\%]$ & Conversion [\%] & Error [\%] \\
\hline 20 & 1.42 & 1.8 & 1.42 & 0.8 & 1.39 & 0.6 \\
\hline 50 & 3.08 & 4.4 & 4.46 & 0.7 & 3.91 & 0.8 \\
\hline 75 & 7.72 & 4.0 & 6.34 & 0.7 & 9.81 & 2.1 \\
\hline 100 & 7.57 & 5.8 & 9.35 & 1.9 & 11.77 & 2.1 \\
\hline 150 & 8.85 & 4.9 & 12.68 & 2.4 & 17.36 & 1.1 \\
\hline 200 & 14.75 & 5.9 & 14.93 & 1.3 & 22.21 & 4.13 \\
\hline 500 & 21.97 & 2.0 & 31.32 & 1.2 & 52.84 & 3.2 \\
\hline 1000 & 39.50 & 6.2 & 47.74 & 1.6 & 69.74 & 4.25 \\
\hline 2000 & 61.50 & 12.0 & 78.62 & 4.0 & 92.52 & 1.0 \\
\hline
\end{tabular}

Table S1-F. Conversion of cyclodextrin with $\mathrm{Mg}^{2+}$ catalyst at $400{ }^{\circ} \mathrm{C}$

\begin{tabular}{|c|c|c|c|c|c|c|}
\hline Temperature $\left[{ }^{\circ} \mathbf{C}\right]$ & \multicolumn{6}{|c|}{400} \\
\hline $\begin{array}{c}\text { Magnesium Concentration } \\
{\left[\mathrm{mmol} \mathrm{Mg}^{2+} / \mathrm{g}-\mathrm{CD}\right]}\end{array}$ & \multicolumn{2}{|c|}{0.3} & \multicolumn{2}{|c|}{0.4} & \multicolumn{2}{|l|}{0.5} \\
\hline Pulse Length [ms] & $\begin{array}{c}\text { Conversion } \\
{[\%]}\end{array}$ & Error [\%] & $\begin{array}{c}\text { Conversion } \\
{[\%]}\end{array}$ & Error [\%] & Conversion [\%] & Error [\%] \\
\hline 20 & 5.34 & 3.9 & 6.65 & 2.4 & 15.20 & 3.3 \\
\hline 50 & 12.52 & 5.4 & 12.61 & 1.7 & 25.96 & 1.3 \\
\hline 75 & 15.37 & 3.8 & 13.14 & 2.7 & 36.88 & 4.2 \\
\hline 100 & 21.55 & 2.8 & 20.88 & 3.3 & 44.24 & 1.3 \\
\hline 150 & 27.08 & 3.3 & 28.55 & 1.6 & 53.46 & 3.5 \\
\hline 200 & 36.57 & 5.9 & 42.22 & 6.8 & 60.36 & 2.1 \\
\hline 500 & 62.91 & 5.1 & 71.92 & 3.8 & 84.82 & 3.4 \\
\hline 1000 & 78.87 & 3.3 & 93.64 & 4.3 & 91.89 & 4.2 \\
\hline 2000 & 96.03 & 3.0 & 98.76 & 1.1 & 97.97 & 2.4 \\
\hline
\end{tabular}


Table S1-G. Conversion of cyclodextrin with $\mathrm{Mg}^{2+}$ catalyst at $407^{\circ} \mathrm{C}$

\begin{tabular}{|c|c|c|c|c|c|c|}
\hline Temperature $\left[{ }^{\circ} \mathbf{C}\right]$ & \multicolumn{6}{|c|}{407} \\
\hline Magnesium Concentration & \multicolumn{2}{|c|}{ Pure CD } & \multicolumn{2}{|c|}{0.1} & \multicolumn{2}{|l|}{0.2} \\
\hline Pulse Length [ms] & $\begin{array}{c}\text { Conversion } \\
{[\%]}\end{array}$ & Error [\%] & $\begin{array}{c}\text { Conversion } \\
{[\%]}\end{array}$ & Error $[\%]$ & Conversion [\%] & Error [\%] \\
\hline 20 & 2.31 & 2.5 & 1.91 & 1.8 & 3.28 & 1.3 \\
\hline 50 & 3.88 & 2.6 & 5.12 & 1.7 & 8.29 & 1.6 \\
\hline 75 & 5.24 & 3.4 & 7.43 & 2.2 & 13.30 & 2.5 \\
\hline 100 & 6.96 & 1.7 & 10.41 & 2.2 & 17.15 & 1.7 \\
\hline 150 & 9.74 & 2.4 & 17.64 & 3.1 & 22.67 & 1.8 \\
\hline 200 & 13.53 & 2.2 & 23.65 & 3.6 & 31.92 & 2.4 \\
\hline 500 & 29.32 & 3.5 & 47.60 & 4.3 & 59.31 & 1.2 \\
\hline 1000 & 52.07 & 2.5 & 63.00 & 2.5 & 83.54 & 3.0 \\
\hline 2000 & 74.50 & 4.5 & 87.81 & 4.1 & 96.91 & 1.6 \\
\hline
\end{tabular}

Table S1-H. Conversion of cyclodextrin with $\mathrm{Mg}^{2+}$ catalyst at $407{ }^{\circ} \mathrm{C}$

\begin{tabular}{|c|c|c|c|c|c|c|}
\hline Temperature $\left[{ }^{\circ} \mathbf{C}\right]$ & \multicolumn{6}{|c|}{407} \\
\hline $\begin{array}{l}\text { Magnesium Concentration } \\
{\left[\mathrm{mmol} \mathrm{Mg}^{2+} / \mathrm{g}_{-} \mathrm{CD}\right]}\end{array}$ & \multicolumn{2}{|c|}{0.3} & \multicolumn{2}{|c|}{0.4} & \multicolumn{2}{|l|}{0.5} \\
\hline Pulse Length [ms] & $\begin{array}{c}\text { Conversion } \\
{[\%]}\end{array}$ & Error $[\%]$ & $\begin{array}{c}\text { Conversion } \\
{[\%]}\end{array}$ & Error [\%] & Conversion [\%] & Error [\%] \\
\hline 20 & 8.62 & 1.7 & 10.80 & 1.9 & 10.81 & 2.1 \\
\hline 50 & 17.24 & 0.7 & 23.15 & 1.6 & 28.57 & 1.2 \\
\hline 75 & 25.87 & 4.9 & 29.70 & 1.8 & 40.29 & 2.1 \\
\hline 100 & 37.89 & 1.1 & 36.97 & 1.5 & 46.12 & 1.8 \\
\hline 150 & 49.92 & 1.3 & 56.74 & 3.1 & 59.02 & 3.2 \\
\hline 200 & 58.84 & 1.2 & 64.42 & 1.8 & 76.81 & 3.3 \\
\hline 500 & 81.27 & 2.1 & 89.12 & 3.8 & 95.40 & 1.9 \\
\hline 1000 & 95.18 & 1.8 & 98.97 & 1.0 & 99.27 & 0.7 \\
\hline 2000 & 98.94 & 1.2 & 99.58 & 0.8 & 99.99 & 0.1 \\
\hline
\end{tabular}


Table S1-I. Conversion of cyclodextrin with $\mathrm{Mg}^{2+}$ catalyst at $415^{\circ} \mathrm{C}$

\begin{tabular}{|c|c|c|c|c|c|c|}
\hline Temperature $\left[{ }^{\circ} \mathbf{C}\right]$ & \multicolumn{6}{|c|}{415} \\
\hline Magnesium Concentration & \multicolumn{2}{|c|}{ Pure CD } & \multicolumn{2}{|c|}{0.1} & \multicolumn{2}{|l|}{0.2} \\
\hline Pulse Length [ms] & $\begin{array}{c}\text { Conversion } \\
{[\%]}\end{array}$ & Error $[\%]$ & $\begin{array}{c}\text { Conversion } \\
{[\%]}\end{array}$ & Error $[\%]$ & Conversion [\%] & Error $[\%]$ \\
\hline 20 & 5.96 & 3.2 & 4.43 & 0.9 & 8.05 & 2.2 \\
\hline 50 & 12.88 & 0.3 & 6.97 & 1.3 & 13.53 & 3.7 \\
\hline 75 & 22.67 & 5.2 & 8.28 & 2.6 & 18.16 & 4.3 \\
\hline 100 & 25.64 & 7.9 & 10.62 & 1.3 & 23.14 & 2.3 \\
\hline 150 & 24.88 & 4.9 & 19.09 & 3.6 & 32.22 & 2.9 \\
\hline 200 & 23.66 & 2.0 & 23.80 & 1.2 & 43.96 & 4.8 \\
\hline 500 & 33.80 & 7.8 & 47.98 & 1.5 & 80.58 & 5.9 \\
\hline 1000 & 55.12 & 4.8 & 72.95 & 2.7 & 92.30 & 6.7 \\
\hline 2000 & 76.00 & 2.4 & 94.63 & 3.3 & 96.56 & 0.7 \\
\hline
\end{tabular}

Table S1-J. Conversion of cyclodextrin with $\mathrm{Mg}^{2+}$ catalyst at $415^{\circ} \mathrm{C}$

\begin{tabular}{|c|c|c|c|c|c|c|}
\hline Temperature $\left[{ }^{\circ} \mathbf{C}\right]$ & \multicolumn{6}{|c|}{415} \\
\hline $\begin{array}{c}\text { Magnesium Concentration } \\
{\left[\mathrm{mmol} \mathrm{Mg}^{2+} / \mathrm{g}-\mathrm{CD}\right]}\end{array}$ & \multicolumn{2}{|c|}{0.3} & \multicolumn{2}{|c|}{0.4} & \multicolumn{2}{|l|}{0.5} \\
\hline Pulse Length [ms] & $\begin{array}{c}\text { Conversion } \\
{[\%]}\end{array}$ & Error [\%] & $\begin{array}{c}\text { Conversion } \\
{[\%]}\end{array}$ & Error [\%] & Conversion [\%] & Error [\%] \\
\hline 20 & 13.94 & 1.3 & 7.92 & 3.6 & 25.13 & 7.7 \\
\hline 50 & 20.33 & 2.2 & 27.59 & 5.8 & 57.48 & 7.0 \\
\hline 75 & 30.07 & 5.5 & 51.30 & 4.6 & 67.86 & 2.9 \\
\hline 100 & 35.56 & 2.1 & 63.62 & 1.3 & 76.17 & 2.66 \\
\hline 150 & 55.94 & 1.5 & 76.57 & 3.5 & 82.23 & 3.0 \\
\hline 200 & 63.89 & 5.4 & 84.70 & 4.8 & 90.02 & 2.5 \\
\hline 500 & 95.29 & 0.8 & 97.55 & 3.6 & 98.97 & 0.5 \\
\hline 1000 & 93.63 & 5.7 & 99.19 & 0.9 & 98.60 & 1.3 \\
\hline 2000 & 96.60 & 2.2 & 97.35 & 4.54 & 99.33 & 0.6 \\
\hline
\end{tabular}


Table S1-K. Conversion of cyclodextrin with $\mathrm{Mg}^{2+}$ catalyst at $430{ }^{\circ} \mathrm{C}$

\begin{tabular}{|c|c|c|c|c|c|c|}
\hline Temperature $\left[{ }^{\circ} \mathbf{C}\right]$ & \multicolumn{6}{|c|}{430} \\
\hline Magnesium Concentration & \multicolumn{2}{|c|}{ Pure CD } & \multicolumn{2}{|c|}{0.1} & \multicolumn{2}{|l|}{0.2} \\
\hline Pulse Length [ms] & $\begin{array}{c}\text { Conversion } \\
{[\%]}\end{array}$ & Error [\%] & $\begin{array}{c}\text { Conversion } \\
{[\%]}\end{array}$ & Error [\%] & Conversion [\%] & Error [\%] \\
\hline 20 & 2.82 & 3.2 & 4.66 & 1.2 & 16.15 & 8.2 \\
\hline 50 & 9.57 & 4.6 & 11.28 & 2.6 & 31.71 & 4.2 \\
\hline 75 & 9.61 & 4.6 & 19.90 & 2.9 & 40.27 & 1.4 \\
\hline 100 & 13.03 & 2.1 & 29.53 & 6.9 & 48.07 & 6.8 \\
\hline 150 & 18.78 & 3.7 & 30.19 & 2.2 & 60.18 & 1.8 \\
\hline 200 & 22.36 & 8.0 & 40.24 & 4.2 & 70.41 & 1.6 \\
\hline 500 & 52.36 & 5.2 & 67.68 & 4.5 & 91.75 & 3.7 \\
\hline 1000 & 75.60 & 5.5 & 90.38 & 3.4 & 93.34 & 7.7 \\
\hline 2000 & 94.06 & 5.3 & 94.73 & 2.5 & 99.35 & 1.3 \\
\hline
\end{tabular}

Table S1-L. Conversion of cyclodextrin with $\mathrm{Mg}^{2+}$ catalyst at $430{ }^{\circ} \mathrm{C}$

\begin{tabular}{|c|c|c|c|c|c|c|}
\hline Temperature $\left[{ }^{\circ} \mathbf{C}\right]$ & \multicolumn{6}{|c|}{430} \\
\hline $\begin{array}{c}\text { Magnesium Concentration } \\
{\left[\mathrm{mmol} \mathrm{Mg}^{2+} / \mathrm{g}-\mathrm{CD}\right]}\end{array}$ & \multicolumn{2}{|c|}{0.3} & \multicolumn{2}{|c|}{0.4} & \multicolumn{2}{|l|}{0.5} \\
\hline Pulse Length [ms] & $\begin{array}{c}\text { Conversion } \\
{[\%]}\end{array}$ & Error [\%] & $\begin{array}{c}\text { Conversion } \\
{[\%]}\end{array}$ & Error [\%] & Conversion [\%] & Error [\%] \\
\hline 20 & 16.19 & 2.7 & 27.45 & 3.7 & 36.68 & 3.9 \\
\hline 50 & 38.87 & 7.9 & 56.79 & 2.8 & 68.21 & 3.6 \\
\hline 75 & 44.57 & 4.2 & 73.23 & 4.7 & 81.16 & 3.2 \\
\hline 100 & 55.03 & 6.7 & 84.34 & 3.3 & 92.25 & 4.5 \\
\hline 150 & 71.41 & 5.8 & 91.32 & 2.6 & 95.26 & 1.5 \\
\hline 200 & 83.56 & 1.9 & 98.44 & 0.8 & 99.40 & 0.2 \\
\hline 500 & 96.25 & 2.5 & 99.61 & 0.1 & 99.55 & 0.3 \\
\hline 1000 & 95.38 & 3.9 & 99.14 & 0.5 & 99.61 & 0.1 \\
\hline 2000 & 97.56 & 1.4 & 99.42 & 0.8 & 99.74 & 0.2 \\
\hline
\end{tabular}




\section{Supplemental Note 2. Additional transition state for dual Mg catalyzed mechanism}

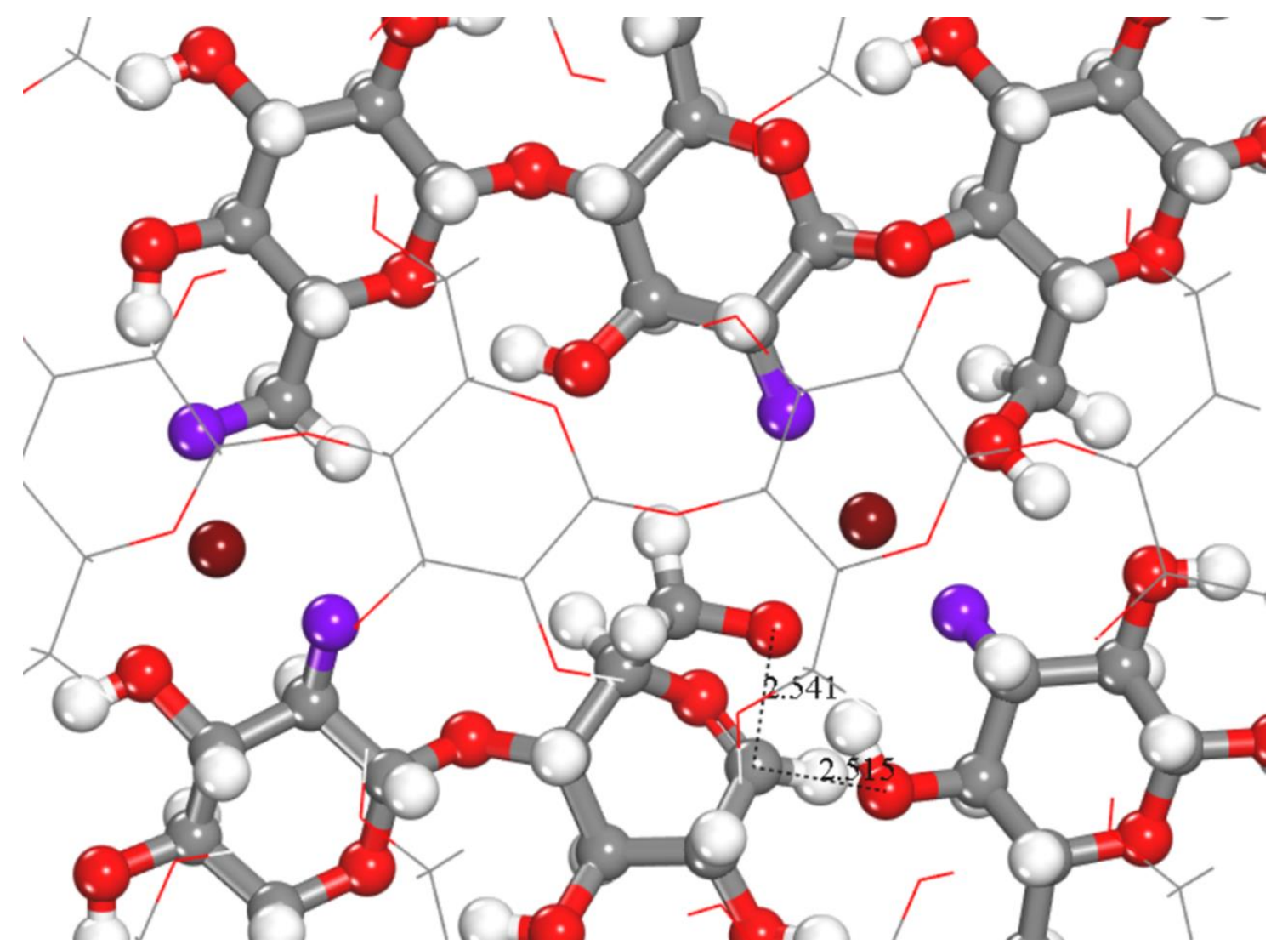

Figure S1: Optimized transition state for dual Mg catalyzed mechanism where one $\mathrm{Mg}$ ion acts as a Lewis acid catalyzed while another $\mathrm{Mg}$ ion helps in H-bond disruption. The calculated barrier is $54 \mathrm{kcal} / \mathrm{mol}$. The higher barrier compared to $\mathrm{Ca}(50 \mathrm{kcal} / \mathrm{mol})$ could be due to higher geometric strain in cellulose chain as it must twist to make a strong interaction with $\mathrm{Mg}$ (shorter $\mathrm{Mg}-\mathrm{O}$ bond). 


\section{Supplemental Note 3. Coordinates for DFT structures}

Reduced coordinates of optimized structures are provided below

Site 01 co-ordinates:

$\mathrm{a}=7.784 \AA, \mathrm{b}=16.402 \AA, \mathrm{c}=20.760 \AA$

$\Upsilon=96.550$

$\mathrm{C} \mathrm{Mg} \mathrm{H} \mathrm{O}$

$\begin{array}{llll}96 & 1 & 158 & 80\end{array}$

\begin{tabular}{|c|c|c|}
\hline $\mathrm{x}$ & $\mathrm{y}$ & $\mathrm{Z}$ \\
\hline 0.011058651 & 0.480988255 & 0.025765985 \\
\hline 0.976799831 & 0.408199188 & 0.482137488 \\
\hline 0.057674794 & 0.430078019 & 0.417108698 \\
\hline 0.004201281 & 0.511725832 & 0.39375685 \\
\hline 0.042508269 & 0.581468906 & 0.443125991 \\
\hline 0.95837224 & 0.658398056 & 0.425428198 \\
\hline 0.510712731 & 0.221593674 & 0.148159302 \\
\hline 0.46030528 & 0.152788408 & 0.100383398 \\
\hline 0.536349146 & 0.177149619 & 0.034909654 \\
\hline 0.48324337 & 0.260778144 & 0.014648797 \\
\hline 0.533021743 & 0.326968839 & 0.065689753 \\
\hline 0.458574191 & 0.407941213 & 0.05358119 \\
\hline 0.000100815 & 0.521693501 & 0.276656519 \\
\hline 0.034104635 & 0.594315442 & 0.230065481 \\
\hline 0.951106659 & 0.568760654 & 0.165304528 \\
\hline 0.018521849 & 0.489727374 & 0.141616275 \\
\hline 0.982965508 & 0.420878943 & 0.191010294 \\
\hline 0.054428015 & 0.340618588 & 0.174004199 \\
\hline 0.471791434 & 0.272014299 & 0.399444918 \\
\hline 0.492230193 & 0.341181652 & 0.34919041 \\
\hline 0.420244093 & 0.304472416 & 0.285554493 \\
\hline 0.502699592 & 0.230819963 & 0.263634066 \\
\hline 0.469040828 & 0.16559913 & 0.31625979 \\
\hline 0.554853921 & 0.088673959 & 0.30277093 \\
\hline 0.01501867 & 0.980498031 & 0.027079813 \\
\hline 0.971668293 & 0.906442839 & 0.480574906 \\
\hline 0.051109875 & 0.930616451 & 0.415405498 \\
\hline 0.988400878 & 0.011404018 & 0.393651976 \\
\hline 0.029075493 & 0.07944235 & 0.444009354 \\
\hline 0.953634731 & 0.158898154 & 0.427841581 \\
\hline 0.510086153 & 0.729546405 & 0.150896006 \\
\hline 0.45057196 & 0.658217833 & 0.105518564 \\
\hline
\end{tabular}




\begin{tabular}{|c|c|c|}
\hline 0.531886281 & 0.677971037 & 0.039637682 \\
\hline 0.485528005 & 0.761544556 & 0.016581362 \\
\hline 0.53934186 & 0.830400479 & 0.064861804 \\
\hline 0.463727508 & 0.909933006 & 0.048648547 \\
\hline 0.990911333 & 0.020126505 & 0.277300315 \\
\hline 0.033910529 & 0.092141252 & 0.230746877 \\
\hline 0.953040136 & 0.068576623 & 0.165753675 \\
\hline 0.016881878 & 0.988320182 & 0.143350198 \\
\hline 0.977338491 & 0.919571452 & 0.19322798 \\
\hline 0.054008234 & 0.840637349 & 0.176226633 \\
\hline 0.494745002 & 0.7750035 & 0.40049977 \\
\hline 0.545063461 & 0.846683668 & 0.354578254 \\
\hline 0.466856446 & 0.82554715 & 0.288683795 \\
\hline 0.515185936 & 0.742643183 & 0.266480792 \\
\hline 0.471873362 & 0.675019559 & 0.316357632 \\
\hline 0.543544196 & 0.59588382 & 0.296121094 \\
\hline 0.023063934 & 0.480060696 & 0.528030361 \\
\hline 0.96751061 & 0.408545964 & 0.979854588 \\
\hline 0.046902583 & 0.432183434 & 0.914756429 \\
\hline 0.983743437 & 0.513020487 & 0.892922257 \\
\hline 0.02307571 & 0.58180126 & 0.942761583 \\
\hline 0.946105858 & 0.660848136 & 0.926257076 \\
\hline 0.516140874 & 0.230016739 & 0.648222197 \\
\hline 0.463329094 & 0.160524632 & 0.600756329 \\
\hline 0.541190264 & 0.183809218 & 0.535140013 \\
\hline 0.486128936 & 0.266871226 & 0.514816218 \\
\hline 0.540351361 & 0.333487145 & 0.564931897 \\
\hline 0.47201394 & 0.414980982 & 0.549489666 \\
\hline 0.987311544 & 0.520470208 & 0.776937388 \\
\hline 0.028589812 & 0.592634333 & 0.730542208 \\
\hline 0.951815181 & 0.568285658 & 0.665263121 \\
\hline 0.019072695 & 0.488408201 & 0.643995282 \\
\hline 0.977335661 & 0.419379582 & 0.693417573 \\
\hline 0.05565237 & 0.340501657 & 0.677280406 \\
\hline 0.485065166 & 0.271042932 & 0.898588584 \\
\hline 0.5401171 & 0.341548033 & 0.852304585 \\
\hline 0.464485748 & 0.320045711 & 0.786110861 \\
\hline 0.51556409 & 0.23674013 & 0.764423631 \\
\hline 0.462956827 & 0.169061694 & 0.813945117 \\
\hline 0.538164691 & 0.088757356 & 0.79997468 \\
\hline 0.012997187 & 0.978286503 & 0.527371761 \\
\hline 0.972348986 & 0.908551073 & 0.98050528 \\
\hline 0.050338673 & 0.932347921 & 0.915133694 \\
\hline
\end{tabular}




\begin{tabular}{|c|c|c|}
\hline 0.986847963 & 0.01300817 & 0.893419497 \\
\hline 0.028058331 & 0.081718278 & 0.943207724 \\
\hline 0.94987657 & 0.160589782 & 0.926999145 \\
\hline 0.51400003 & 0.728631099 & 0.649985204 \\
\hline 0.459079848 & 0.659183929 & 0.602757893 \\
\hline 0.537736148 & 0.681911628 & 0.537265553 \\
\hline 0.488552373 & 0.765714308 & 0.516438045 \\
\hline 0.538602657 & 0.83244358 & 0.566818421 \\
\hline 0.462501811 & 0.912458603 & 0.552955145 \\
\hline 0.987357346 & 0.020140284 & 0.777083606 \\
\hline 0.028863304 & 0.091835979 & 0.73025251 \\
\hline 0.950705085 & 0.067595592 & 0.664987979 \\
\hline 0.014029157 & 0.986741777 & 0.643558084 \\
\hline 0.973915766 & 0.918455212 & 0.693752495 \\
\hline 0.051829033 & 0.839502942 & 0.677624607 \\
\hline 0.485611094 & 0.769658595 & 0.900337924 \\
\hline 0.541356568 & 0.840092393 & 0.85384913 \\
\hline 0.464200856 & 0.818952227 & 0.787950272 \\
\hline 0.514289777 & 0.735711046 & 0.766141013 \\
\hline 0.460987809 & 0.667827559 & 0.815426862 \\
\hline 0.536275336 & 0.587796691 & 0.800781253 \\
\hline 0.315849296 & 0.457174552 & 0.289440775 \\
\hline 0.153440562 & 0.494616268 & 0.031300405 \\
\hline 0.834275259 & 0.40000165 & 0.477849745 \\
\hline 0.199723171 & 0.432987521 & 0.420287885 \\
\hline 0.863354103 & 0.504757762 & 0.384593273 \\
\hline 0.183912204 & 0.596364619 & 0.449734529 \\
\hline 0.951942568 & 0.696324105 & 0.468968826 \\
\hline 0.824880593 & 0.640015393 & 0.40861207 \\
\hline 0.99092725 & 0.325537845 & 0.04804339 \\
\hline 0.88238554 & 0.358053409 & 0.368065181 \\
\hline 0.034272336 & 0.764306879 & 0.38130288 \\
\hline 0.652505438 & 0.230997813 & 0.155644704 \\
\hline 0.317161632 & 0.144842253 & 0.097027917 \\
\hline 0.679317308 & 0.182618635 & 0.038456835 \\
\hline 0.34168028 & 0.255585858 & 0.007116402 \\
\hline 0.675131447 & 0.337135327 & 0.070909414 \\
\hline 0.477271608 & 0.445689656 & 0.097499224 \\
\hline 0.31852412 & 0.393252909 & 0.044323553 \\
\hline 0.499313105 & 0.072673421 & 0.16924173 \\
\hline 0.520450773 & 0.139041255 & 0.446512239 \\
\hline 0.526961313 & 0.511605401 & 0.006070974 \\
\hline 0.860176445 & 0.506948475 & 0.28611039 \\
\hline
\end{tabular}




\begin{tabular}{|c|c|c|}
\hline 0.175654301 & 0.6075371 & 0.223815777 \\
\hline 0.808698359 & 0.557219925 & 0.170609833 \\
\hline 0.159911724 & 0.501725428 & 0.133051475 \\
\hline 0.841985962 & 0.409272846 & 0.199891849 \\
\hline 0.064417425 & 0.305166192 & 0.218742299 \\
\hline 0.184760829 & 0.354536213 & 0.153168291 \\
\hline 0.004653829 & 0.676864732 & 0.298572822 \\
\hline 0.956036736 & 0.613700712 & 0.07651511 \\
\hline 0.965760512 & 0.234683285 & 0.133070042 \\
\hline 0.333265954 & 0.253803444 & 0.409446763 \\
\hline 0.636026475 & 0.357222029 & 0.342761725 \\
\hline 0.280670553 & 0.284352516 & 0.293282883 \\
\hline 0.643636926 & 0.244937239 & 0.256328709 \\
\hline 0.328282461 & 0.150335663 & 0.323036857 \\
\hline 0.542465313 & 0.048927345 & 0.34563802 \\
\hline 0.693063067 & 0.107716587 & 0.292878799 \\
\hline 0.435605819 & 0.349853328 & 0.194613434 \\
\hline 0.485796019 & 0.985533752 & 0.253964827 \\
\hline 0.157072143 & 0.993440235 & 0.033725834 \\
\hline 0.829689697 & 0.895187814 & 0.475746869 \\
\hline 0.193747467 & 0.93931636 & 0.419283231 \\
\hline 0.84663732 & 0.001695712 & 0.385989229 \\
\hline 0.171055964 & 0.091852901 & 0.450672355 \\
\hline 0.955018193 & 0.19671375 & 0.471750111 \\
\hline 0.818223694 & 0.144565097 & 0.411886564 \\
\hline $4.68704 \mathrm{E}-05$ & 0.825942867 & 0.048756367 \\
\hline 0.038186277 & 0.886264802 & 0.326349258 \\
\hline 0.043293435 & 0.263556679 & 0.384567201 \\
\hline 0.652180609 & 0.734053113 & 0.15745179 \\
\hline 0.307800738 & 0.654499048 & 0.101163497 \\
\hline 0.674407784 & 0.681353903 & 0.044368939 \\
\hline 0.344238945 & 0.75813751 & 0.008851258 \\
\hline 0.681781085 & 0.840878892 & 0.068480679 \\
\hline 0.469958617 & 0.948440482 & 0.092157229 \\
\hline 0.326956677 & 0.893772984 & 0.034858715 \\
\hline 0.471443684 & 0.575842252 & 0.177402324 \\
\hline 0.517598719 & 0.637893441 & 0.448349794 \\
\hline 0.53808494 & 0.013440663 & 0.001910024 \\
\hline 0.84897977 & 0.006500346 & 0.28375868 \\
\hline 0.175962597 & 0.101986431 & 0.22568304 \\
\hline 0.810504266 & 0.059082794 & 0.170174567 \\
\hline 0.158607162 & 0.998584764 & 0.135665193 \\
\hline 0.835498604 & 0.90648324 & 0.199595808 \\
\hline
\end{tabular}




\begin{tabular}{|c|c|c|}
\hline 0.056587403 & 0.802987055 & 0.220113304 \\
\hline 0.188341691 & 0.855497733 & 0.159186929 \\
\hline 0.00699308 & 0.174542675 & 0.29937053 \\
\hline 0.964863074 & 0.113670232 & 0.076852511 \\
\hline 0.968643284 & 0.736009153 & 0.132227651 \\
\hline 0.352391972 & 0.764706945 & 0.406731571 \\
\hline 0.688120216 & 0.854363908 & 0.350950392 \\
\hline 0.324204496 & 0.822157361 & 0.29250285 \\
\hline 0.65581115 & 0.748076407 & 0.256961794 \\
\hline 0.330292009 & 0.663722737 & 0.323318225 \\
\hline 0.556758804 & 0.559662469 & 0.341001317 \\
\hline 0.675690559 & 0.612534447 & 0.277161571 \\
\hline 0.513413758 & 0.927206011 & 0.424534514 \\
\hline 0.497380149 & 0.868490489 & 0.199290113 \\
\hline 0.164870075 & 0.491987405 & 0.535395773 \\
\hline 0.825384106 & 0.397580278 & 0.975314737 \\
\hline 0.189571571 & 0.441170639 & 0.918870156 \\
\hline 0.842088827 & 0.502834775 & 0.884996608 \\
\hline 0.164883841 & 0.594927588 & 0.949261524 \\
\hline 0.944911706 & 0.698258971 & 0.970346227 \\
\hline 0.811343016 & 0.646035107 & 0.909706159 \\
\hline 0.001786732 & 0.325130817 & 0.550386952 \\
\hline 0.0348243 & 0.387420971 & 0.825854248 \\
\hline 0.028032697 & 0.765749926 & 0.881941079 \\
\hline 0.658264656 & 0.237684036 & 0.655196503 \\
\hline 0.32023473 & 0.153401912 & 0.597183207 \\
\hline 0.684093036 & 0.189665885 & 0.539207232 \\
\hline 0.343709625 & 0.261550067 & 0.510152796 \\
\hline 0.682595785 & 0.342591747 & 0.569866004 \\
\hline 0.456200044 & 0.447298936 & 0.595497288 \\
\hline 0.342934194 & 0.401219316 & 0.526984931 \\
\hline 0.492123645 & 0.077078849 & 0.668447554 \\
\hline 0.50852555 & 0.134242096 & 0.945610382 \\
\hline 0.553574666 & 0.51860487 & 0.506988903 \\
\hline 0.845324311 & 0.506698338 & 0.783322993 \\
\hline 0.170596621 & 0.604507576 & 0.726167041 \\
\hline 0.809020308 & 0.558033798 & 0.668291551 \\
\hline 0.16121833 & 0.499215238 & 0.637502869 \\
\hline 0.835191051 & 0.40605752 & 0.698784791 \\
\hline 0.056563676 & 0.303186678 & 0.721417532 \\
\hline 0.190482545 & 0.35567616 & 0.660930829 \\
\hline 0.999033278 & 0.675129256 & 0.798693479 \\
\hline 0.967369173 & 0.613073567 & 0.57628132 \\
\hline
\end{tabular}




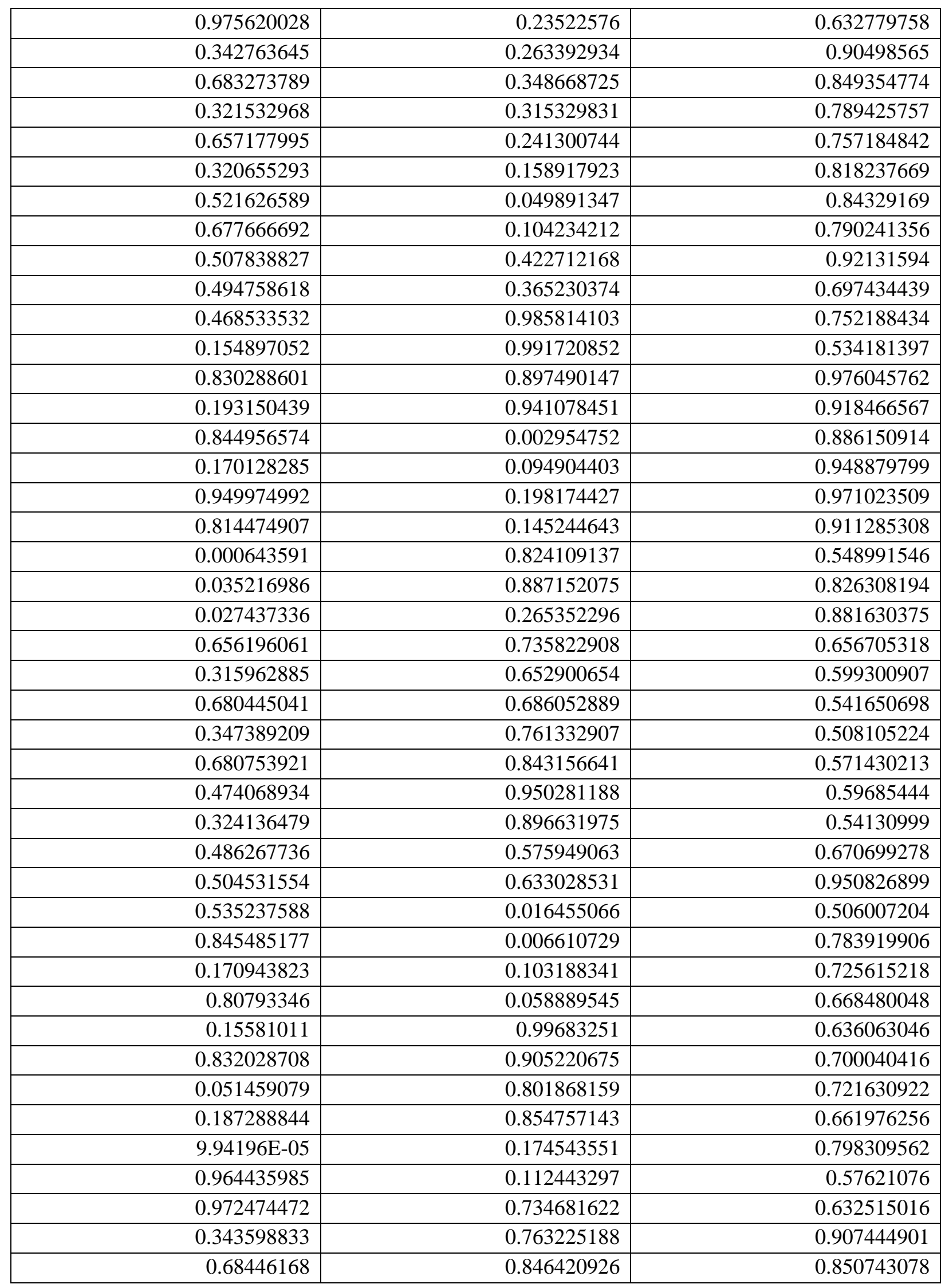




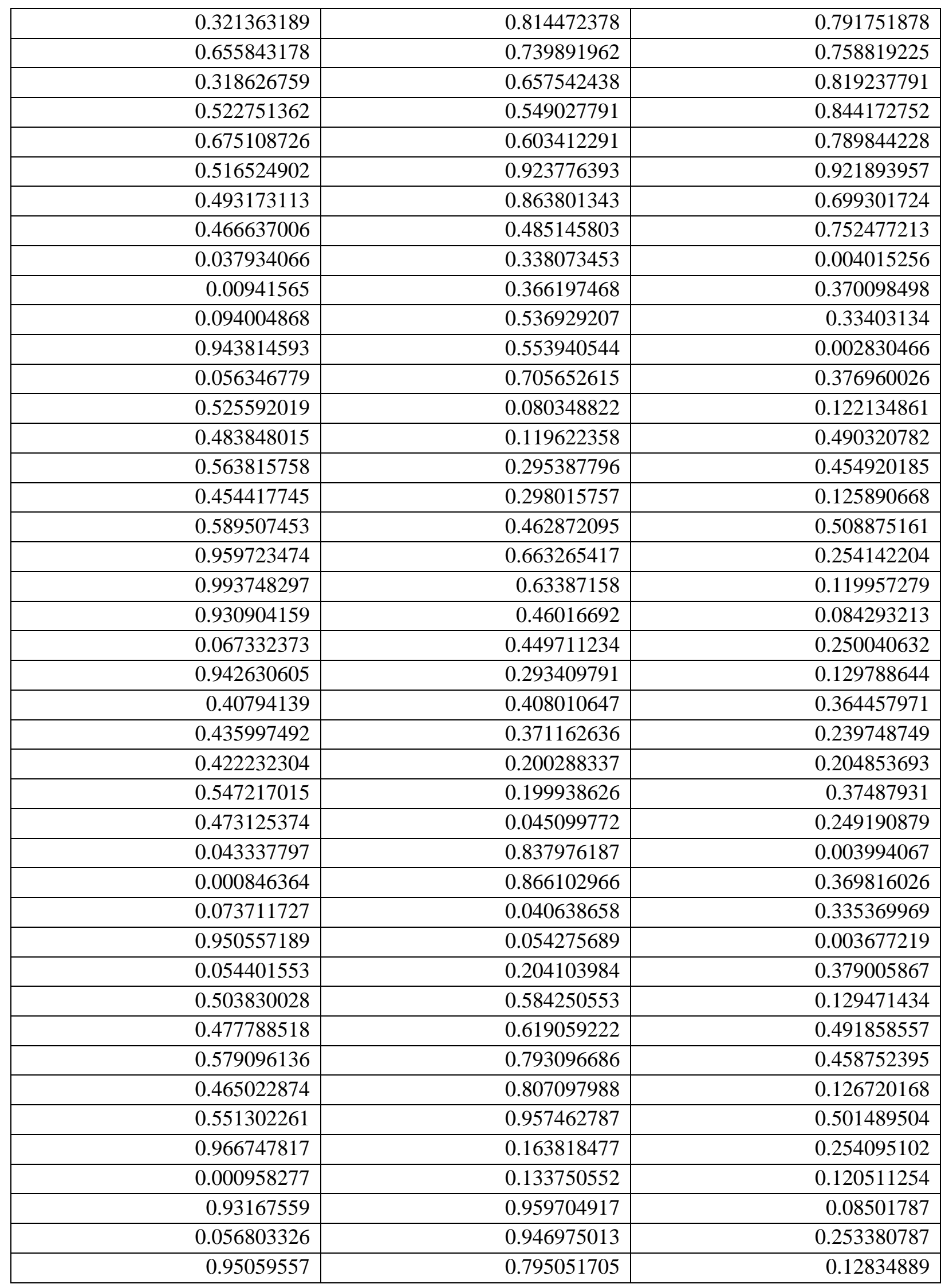




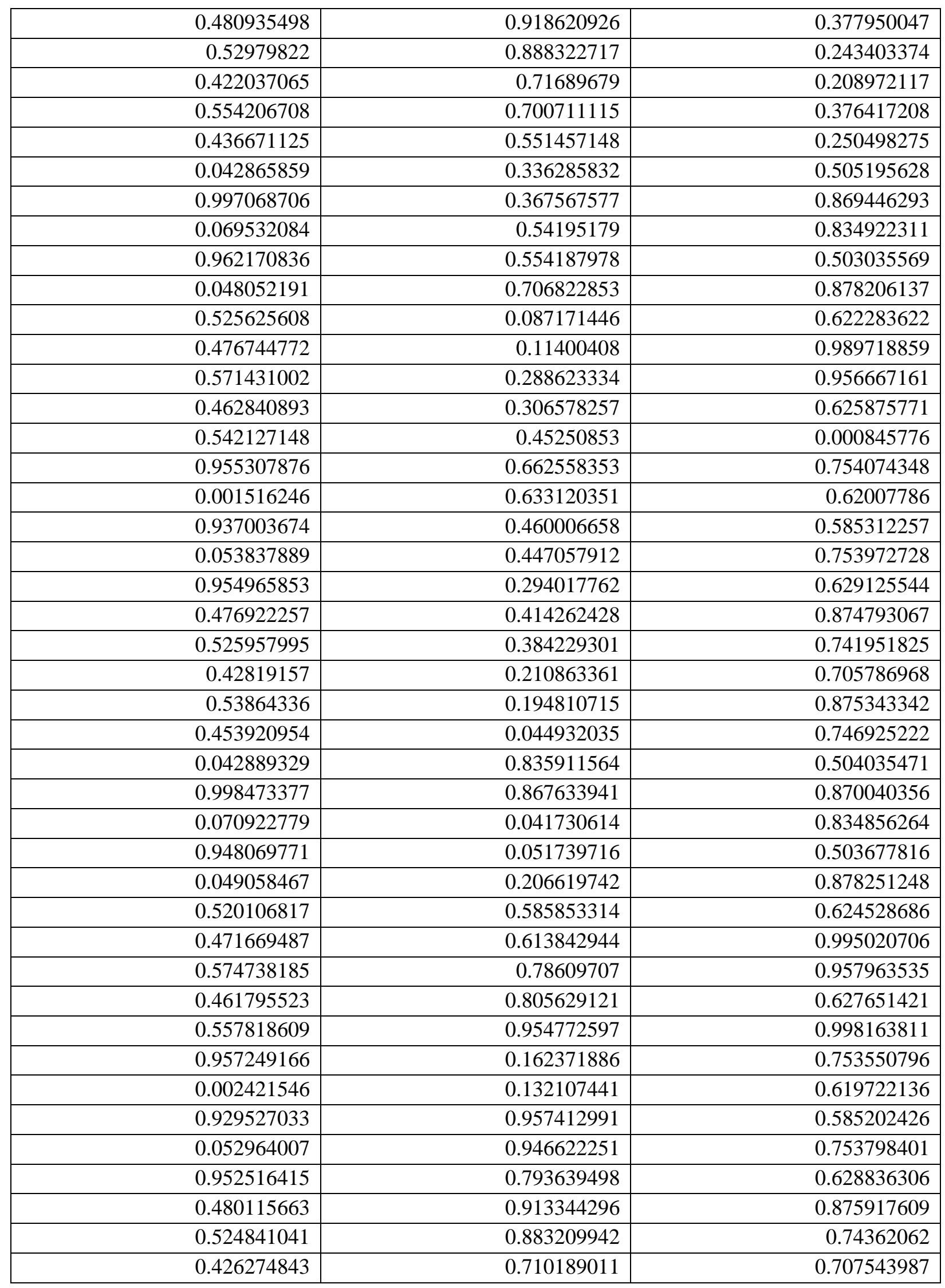




\begin{tabular}{|r|r|r|}
\hline 0.535455338 & 0.692737325 & 0.87708289 \\
\hline 0.448568914 & 0.544021911 & 0.748470683 \\
\hline
\end{tabular}

Site 02 co-ordinates:

$\mathrm{a}=7.784 \AA, \mathrm{b}=16.402 \AA, \mathrm{c}=20.760 \AA$

$\Upsilon=96.550$

$\mathrm{C} \mathrm{Mg} \mathrm{H} \mathrm{O}$

$\begin{array}{lll}961 & 15880\end{array}$

\begin{tabular}{|c|c|c|}
\hline $\mathrm{x}$ & $\mathrm{y}$ & $\mathrm{z}$ \\
\hline 0.013731997 & 0.479732809 & 0.028001577 \\
\hline 0.958542793 & 0.407418879 & 0.481392264 \\
\hline 0.040921204 & 0.433683974 & 0.416951048 \\
\hline 0.972405659 & 0.512966626 & 0.393780866 \\
\hline 0.007189575 & 0.582294017 & 0.443478911 \\
\hline 0.933443784 & 0.662292301 & 0.427991808 \\
\hline 0.51514196 & 0.229183765 & 0.149501127 \\
\hline 0.461176856 & 0.159115764 & 0.102519759 \\
\hline 0.538252379 & 0.181199271 & 0.036724628 \\
\hline 0.486413789 & 0.264351566 & 0.015437694 \\
\hline 0.538244674 & 0.331714055 & 0.065262736 \\
\hline 0.461945086 & 0.411510039 & 0.050802041 \\
\hline 0.985260006 & 0.520892456 & 0.277531406 \\
\hline 0.032423848 & 0.592768961 & 0.231310506 \\
\hline 0.954400244 & 0.568558753 & 0.165966238 \\
\hline 0.017394264 & 0.487760366 & 0.144352159 \\
\hline 0.976979328 & 0.419360462 & 0.19453496 \\
\hline 0.054834932 & 0.340278038 & 0.178712313 \\
\hline 0.487131097 & 0.266425734 & 0.400509711 \\
\hline 0.544551758 & 0.338085827 & 0.355172857 \\
\hline 0.466257325 & 0.318373889 & 0.288989311 \\
\hline 0.514314367 & 0.235439889 & 0.265829076 \\
\hline 0.459640562 & 0.16640104 & 0.313892985 \\
\hline 0.535060072 & 0.086925165 & 0.297731187 \\
\hline 0.013566584 & 0.979284254 & 0.027623341 \\
\hline 0.966686719 & 0.908939854 & 0.48106727 \\
\hline 0.046883037 & 0.932592361 & 0.416030649 \\
\hline 0.983365591 & 0.013032911 & 0.393901662 \\
\hline 0.023748028 & 0.081814789 & 0.443691207 \\
\hline 0.946795018 & 0.160847609 & 0.427110197 \\
\hline 0.513031291 & 0.727755015 & 0.148043758 \\
\hline 0.458753817 & 0.657096649 & 0.101866186 \\
\hline 0.536196427 & 0.678830604 & 0.036065363 \\
\hline
\end{tabular}

Facas et al. 


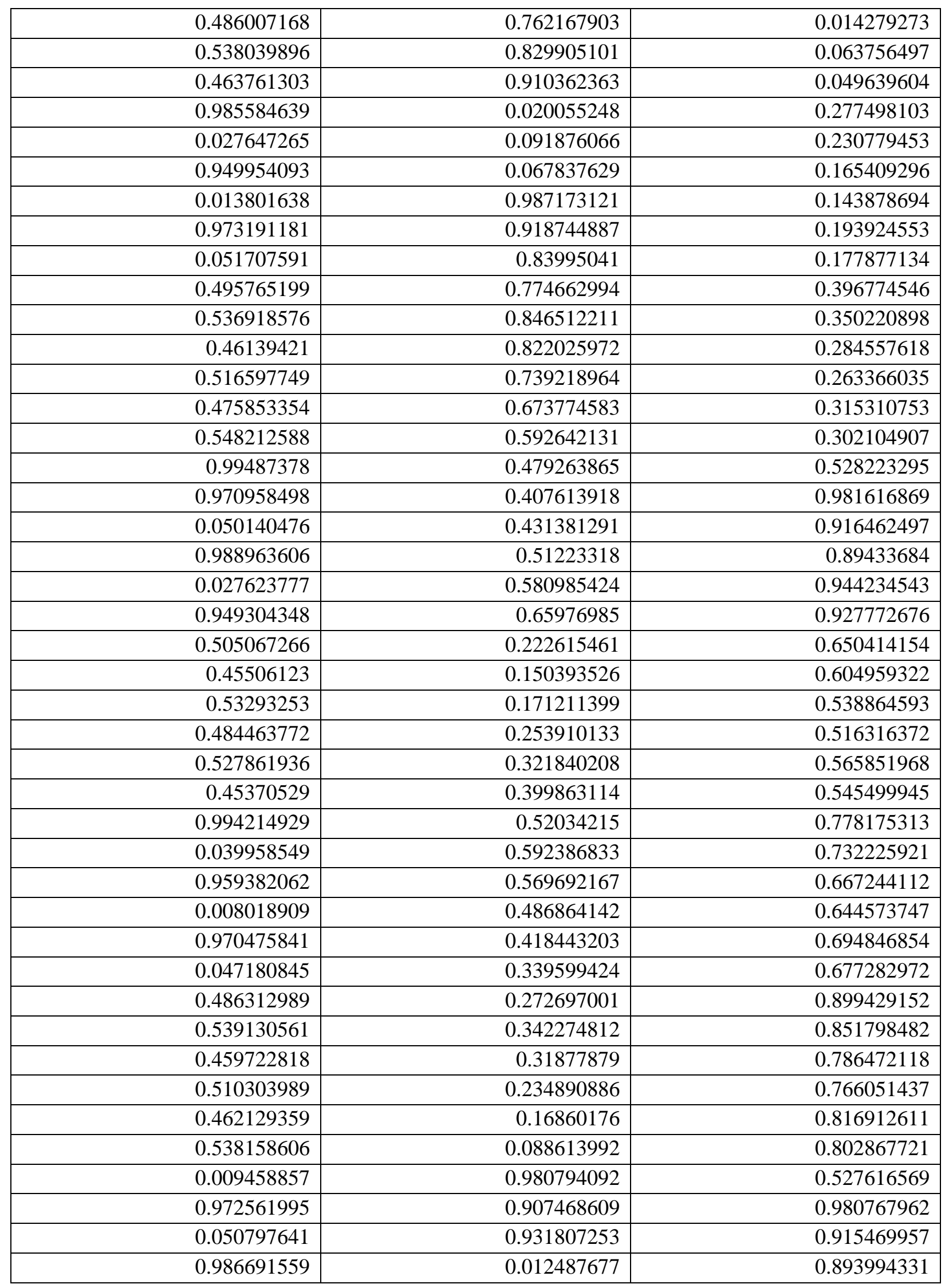




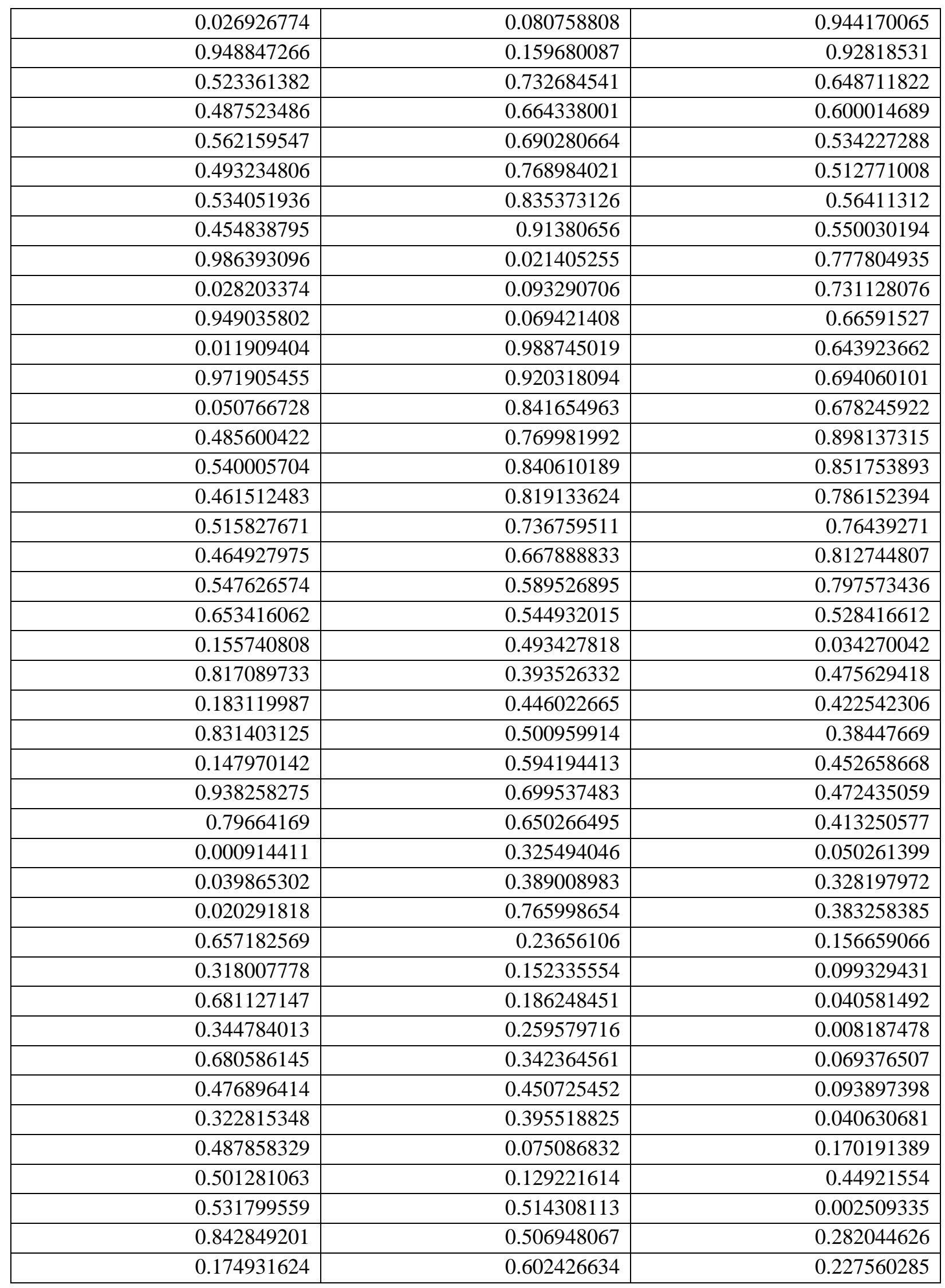




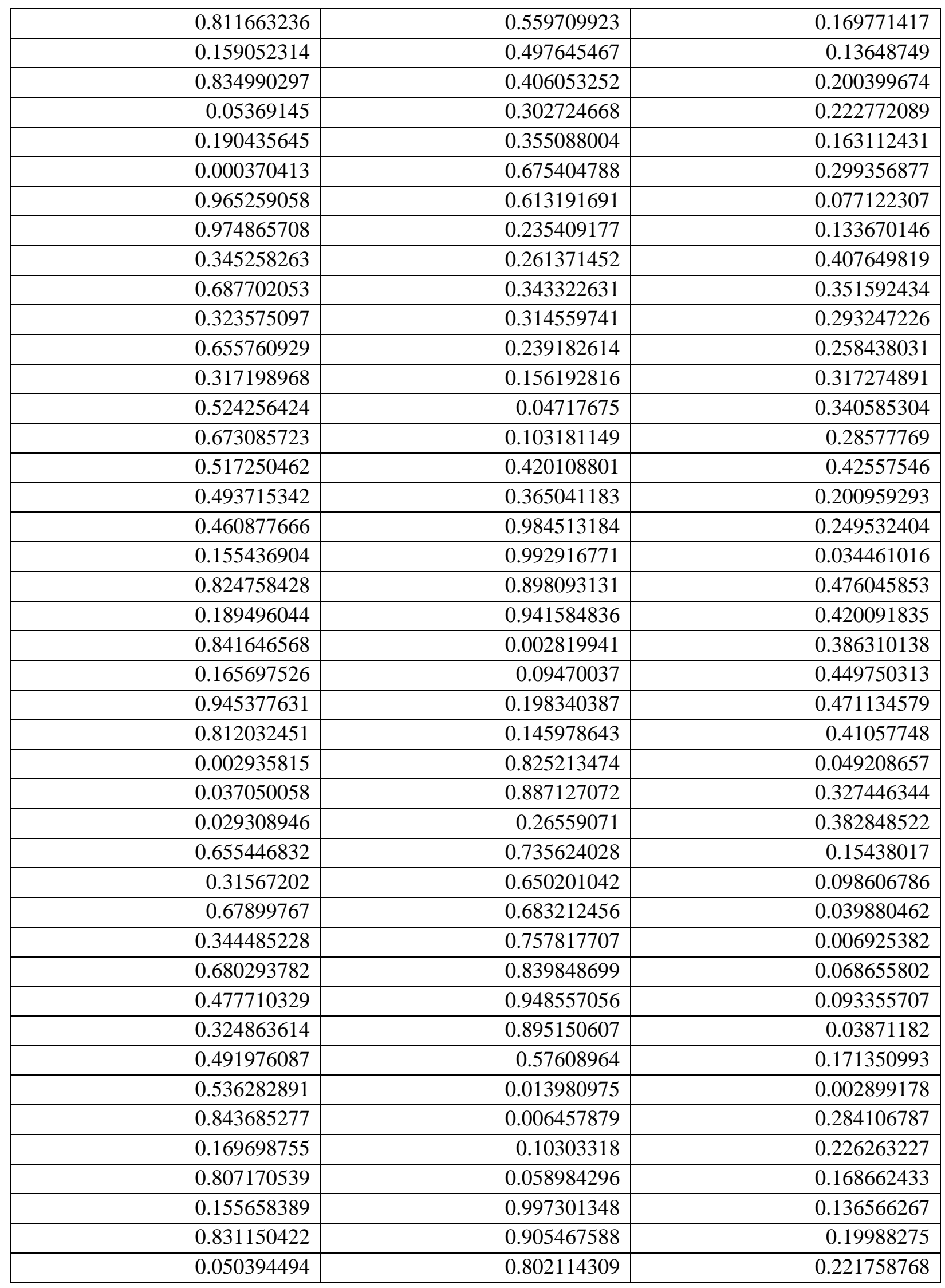




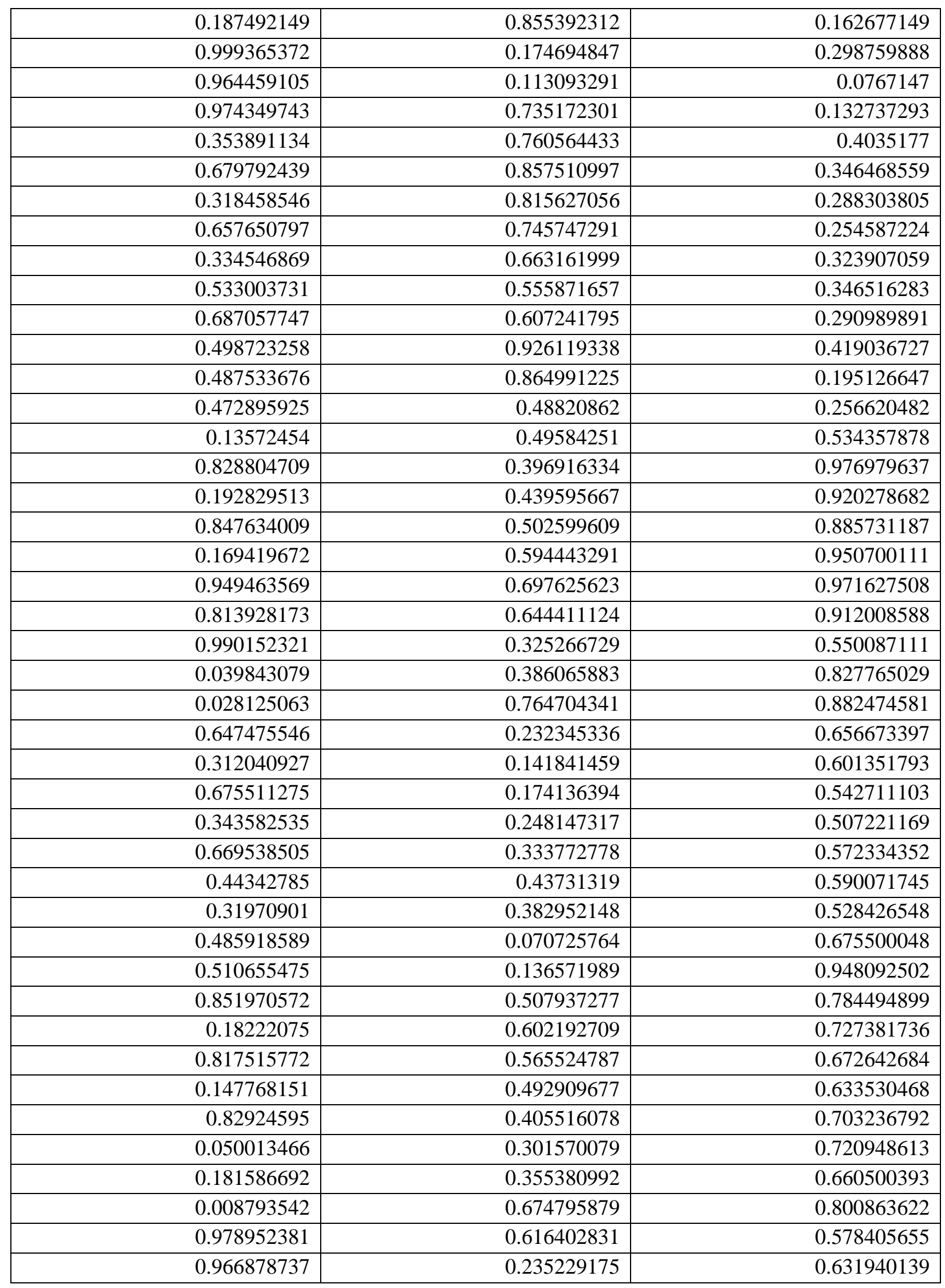




\begin{tabular}{|c|c|c|}
\hline 0.34405188 & 0.264501106 & 0.906126976 \\
\hline 0.682172331 & 0.34901274 & 0.848023926 \\
\hline 0.317068989 & 0.314263544 & 0.790847284 \\
\hline 0.651546247 & 0.239814228 & 0.757756691 \\
\hline 0.320134031 & 0.157657781 & 0.822245795 \\
\hline 0.530312522 & 0.051506895 & 0.847181481 \\
\hline 0.675546397 & 0.104473046 & 0.789736778 \\
\hline 0.509388626 & 0.425059577 & 0.920089317 \\
\hline 0.482474392 & 0.359920501 & 0.696682996 \\
\hline 0.464103288 & 0.983964191 & 0.757204106 \\
\hline 0.15137975 & 0.993872904 & 0.534296193 \\
\hline 0.830525273 & 0.896028318 & 0.976077885 \\
\hline 0.193550916 & 0.940795443 & 0.918981994 \\
\hline 0.844843283 & 0.002189499 & 0.886678866 \\
\hline 0.168901082 & 0.093975104 & 0.950353864 \\
\hline 0.948985344 & 0.197090044 & 0.972292069 \\
\hline 0.813506082 & 0.14437394 & 0.912428611 \\
\hline 0.993873359 & 0.826790534 & 0.549547797 \\
\hline 0.03572624 & 0.887359224 & 0.826446038 \\
\hline 0.026969536 & 0.264735083 & 0.883536856 \\
\hline 0.663961367 & 0.746821679 & 0.65748841 \\
\hline 0.346421727 & 0.647065995 & 0.596736116 \\
\hline 0.704401649 & 0.709056682 & 0.542714358 \\
\hline 0.351677564 & 0.758716229 & 0.50511989 \\
\hline 0.675405076 & 0.848274709 & 0.570429466 \\
\hline 0.467779396 & 0.953729315 & 0.592801789 \\
\hline 0.316356566 & 0.897049877 & 0.539142615 \\
\hline 0.539765933 & 0.575395594 & 0.663347085 \\
\hline 0.508291929 & 0.634275244 & 0.947144423 \\
\hline 0.525238566 & 0.015089291 & 0.500614288 \\
\hline 0.844478949 & 0.008214072 & 0.784740676 \\
\hline 0.170188507 & 0.104299957 & 0.726435159 \\
\hline 0.80640942 & 0.06059074 & 0.669622378 \\
\hline 0.153654339 & 0.998490735 & 0.636208745 \\
\hline 0.829936218 & 0.90699469 & 0.700191888 \\
\hline 0.052900628 & 0.804913221 & 0.722646469 \\
\hline 0.185255288 & 0.857098499 & 0.661604558 \\
\hline 0.999457751 & 0.175247441 & 0.799856966 \\
\hline 0.963891196 & 0.113932407 & 0.57672426 \\
\hline 0.976139433 & 0.73671831 & 0.634037652 \\
\hline 0.343567211 & 0.762501162 & 0.905153567 \\
\hline 0.683095553 & 0.846950072 & 0.848181667 \\
\hline 0.318650484 & 0.813612273 & 0.790264758 \\
\hline
\end{tabular}




\begin{tabular}{|c|c|c|}
\hline 0.657990936 & 0.743018861 & 0.758321695 \\
\hline 0.322743101 & 0.655911249 & 0.816519779 \\
\hline 0.522981504 & 0.546877836 & 0.838271083 \\
\hline 0.688068663 & 0.607772257 & 0.792921772 \\
\hline 0.513767181 & 0.92358268 & 0.920486147 \\
\hline 0.483784047 & 0.864981135 & 0.697496796 \\
\hline 0.480998562 & 0.487673396 & 0.747070181 \\
\hline 0.041409267 & 0.336927771 & 0.005014537 \\
\hline 0.000289283 & 0.368716085 & 0.371449726 \\
\hline 0.062031626 & 0.542151628 & 0.336662541 \\
\hline 0.947648741 & 0.553023283 & 0.004091595 \\
\hline 0.037391356 & 0.706624265 & 0.379859239 \\
\hline 0.52357763 & 0.085983362 & 0.124077312 \\
\hline 0.469299083 & 0.109055868 & 0.493338854 \\
\hline 0.576529671 & 0.278992699 & 0.458537572 \\
\hline 0.463044382 & 0.305939642 & 0.126526324 \\
\hline 0.55744821 & 0.442345233 & 0.498573476 \\
\hline 0.963380218 & 0.664174781 & 0.253750673 \\
\hline 0.004836921 & 0.63291756 & 0.12041324 \\
\hline 0.931496089 & 0.458914372 & 0.086032608 \\
\hline 0.054137681 & 0.44778882 & 0.254894039 \\
\hline 0.955415487 & 0.294374679 & 0.129881862 \\
\hline 0.485607291 & 0.411196849 & 0.378052913 \\
\hline 0.527255766 & 0.383265851 & 0.245229238 \\
\hline 0.42612676 & 0.211403019 & 0.206863117 \\
\hline 0.533113966 & 0.189291308 & 0.376043695 \\
\hline 0.444912155 & 0.043819075 & 0.24561115 \\
\hline 0.044276059 & 0.836999757 & 0.004094862 \\
\hline 0.996982174 & 0.867955748 & 0.3708204 \\
\hline 0.068358694 & 0.041582882 & 0.33544478 \\
\hline 0.947879624 & 0.052713174 & 0.004115161 \\
\hline 0.048637415 & 0.206742861 & 0.378921166 \\
\hline 0.521231023 & 0.584313837 & 0.124480758 \\
\hline 0.533189582 & 0.62603154 & 0.491216097 \\
\hline 0.578091578 & 0.797768125 & 0.454711633 \\
\hline 0.45982921 & 0.804020544 & 0.124418047 \\
\hline 0.542621258 & 0.955952155 & 0.497067906 \\
\hline 0.956377509 & 0.162549589 & 0.254032013 \\
\hline 0.001774856 & 0.132635231 & 0.12031923 \\
\hline 0.929901967 & 0.957989586 & 0.085316693 \\
\hline 0.051594737 & 0.946728988 & 0.254077265 \\
\hline 0.954037396 & 0.793979548 & 0.128628901 \\
\hline 0.465626895 & 0.917291923 & 0.37262365 \\
\hline
\end{tabular}




\begin{tabular}{|c|c|c|}
\hline 0.518015891 & 0.885852594 & 0.239088247 \\
\hline 0.426552713 & 0.710844183 & 0.205733695 \\
\hline 0.564783223 & 0.704279852 & 0.372040129 \\
\hline 0.460280355 & 0.547182289 & 0.250229783 \\
\hline 0.034849157 & 0.339034341 & 0.505721914 \\
\hline 0.998391433 & 0.367039147 & 0.870933195 \\
\hline 0.077192045 & 0.540050419 & 0.836146625 \\
\hline 0.922920716 & 0.552609394 & 0.503978975 \\
\hline 0.048677097 & 0.705774408 & 0.878976376 \\
\hline 0.52150141 & 0.079557986 & 0.629230644 \\
\hline 0.478836366 & 0.11687598 & 0.992264553 \\
\hline 0.573539641 & 0.291514545 & 0.956896081 \\
\hline 0.446861829 & 0.29692816 & 0.626132084 \\
\hline 0.546914012 & 0.455167408 & 0.997732191 \\
\hline 0.970699033 & 0.663378891 & 0.755226592 \\
\hline 0.017432827 & 0.633559344 & 0.622036769 \\
\hline 0.911142003 & 0.461534301 & 0.586778497 \\
\hline 0.056835229 & 0.446595963 & 0.753553695 \\
\hline 0.945144484 & 0.294187247 & 0.629076286 \\
\hline 0.477679973 & 0.415723936 & 0.873490821 \\
\hline 0.517883494 & 0.381349414 & 0.740141269 \\
\hline 0.420130762 & 0.206076823 & 0.708744892 \\
\hline 0.540918985 & 0.196288132 & 0.87706668 \\
\hline 0.445645735 & 0.042727794 & 0.75271398 \\
\hline 0.036670709 & 0.838191822 & 0.504837547 \\
\hline$-2.03303 \mathrm{E}-05$ & 0.867141958 & 0.870080449 \\
\hline 0.070445011 & 0.042091 & 0.835584762 \\
\hline 0.945105785 & 0.054577986 & 0.504116039 \\
\hline 0.047960049 & 0.206031713 & 0.879542619 \\
\hline 0.572388795 & 0.59274735 & 0.617793677 \\
\hline 0.475596206 & 0.615007119 & 0.991320624 \\
\hline 0.574104434 & 0.787411161 & 0.955572498 \\
\hline 0.456100703 & 0.805380087 & 0.624541098 \\
\hline 0.551470346 & 0.954954433 & 0.997621699 \\
\hline 0.957529367 & 0.163913737 & 0.754812787 \\
\hline 0.999337471 & 0.134167042 & 0.620464138 \\
\hline 0.926293183 & 0.960012245 & 0.585704223 \\
\hline 0.050884273 & 0.947953367 & 0.754141195 \\
\hline 0.950104845 & 0.794454149 & 0.630538508 \\
\hline 0.479311398 & 0.913719193 & 0.874246028 \\
\hline 0.519670404 & 0.883430892 & 0.741647806 \\
\hline 0.433004423 & 0.710486561 & 0.705214217 \\
\hline 0.537837872 & 0.693049482 & 0.874647007 \\
\hline
\end{tabular}


Site 03 co-ordinates:

$\mathrm{a}=7.784 \AA, \mathrm{b}=16.402 \AA, \mathrm{c}=20.760 \AA$

$\Upsilon=96.550$

$\mathrm{C} \mathrm{Mg} \mathrm{H} \mathrm{O}$

$\begin{array}{llll}96 & 1 & 158 & 80\end{array}$

\begin{tabular}{|c|c|c|}
\hline $\mathrm{X}$ & $\mathrm{y}$ & $\mathrm{z}$ \\
\hline 0.01231686 & 0.48040612 & 0.027875469 \\
\hline 0.965394379 & 0.406981044 & 0.480863594 \\
\hline 0.045842409 & 0.431160825 & 0.415839113 \\
\hline 0.983936863 & 0.512227701 & 0.394178571 \\
\hline 0.025254841 & 0.580547522 & 0.444319335 \\
\hline 0.948032266 & 0.659805756 & 0.428533803 \\
\hline 0.513012282 & 0.23070559 & 0.142711872 \\
\hline 0.460524149 & 0.161614497 & 0.095166706 \\
\hline 0.536622865 & 0.185162825 & 0.029503797 \\
\hline 0.484821749 & 0.268802665 & 0.009105997 \\
\hline 0.535189752 & 0.334963773 & 0.060033775 \\
\hline 0.459148916 & 0.415265515 & 0.046891801 \\
\hline 0.986127631 & 0.519991651 & 0.277832772 \\
\hline 0.028091662 & 0.592057746 & 0.231332743 \\
\hline 0.950034255 & 0.568236564 & 0.165971069 \\
\hline 0.012736067 & 0.487490403 & 0.144126284 \\
\hline 0.972475094 & 0.418811366 & 0.194000062 \\
\hline 0.050986838 & 0.340011279 & 0.177872386 \\
\hline 0.488011094 & 0.268573911 & 0.392691511 \\
\hline 0.542677654 & 0.33953979 & 0.346627083 \\
\hline 0.465085784 & 0.318346225 & 0.280892205 \\
\hline 0.515228917 & 0.235239586 & 0.258749588 \\
\hline 0.462315363 & 0.167395147 & 0.308238208 \\
\hline 0.53552608 & 0.086653564 & 0.294317472 \\
\hline 0.011571585 & 0.98010254 & 0.027548244 \\
\hline 0.973959989 & 0.907331041 & 0.481193343 \\
\hline 0.050452458 & 0.931387229 & 0.415611418 \\
\hline 0.98654021 & 0.011975976 & 0.393956425 \\
\hline 0.026476689 & 0.080552299 & 0.443924333 \\
\hline 0.947029834 & 0.159081962 & 0.427750711 \\
\hline 0.515990622 & 0.731221498 & 0.151922019 \\
\hline 0.462029194 & 0.662228414 & 0.103992871 \\
\hline 0.539219889 & 0.685585837 & 0.038421543 \\
\hline
\end{tabular}




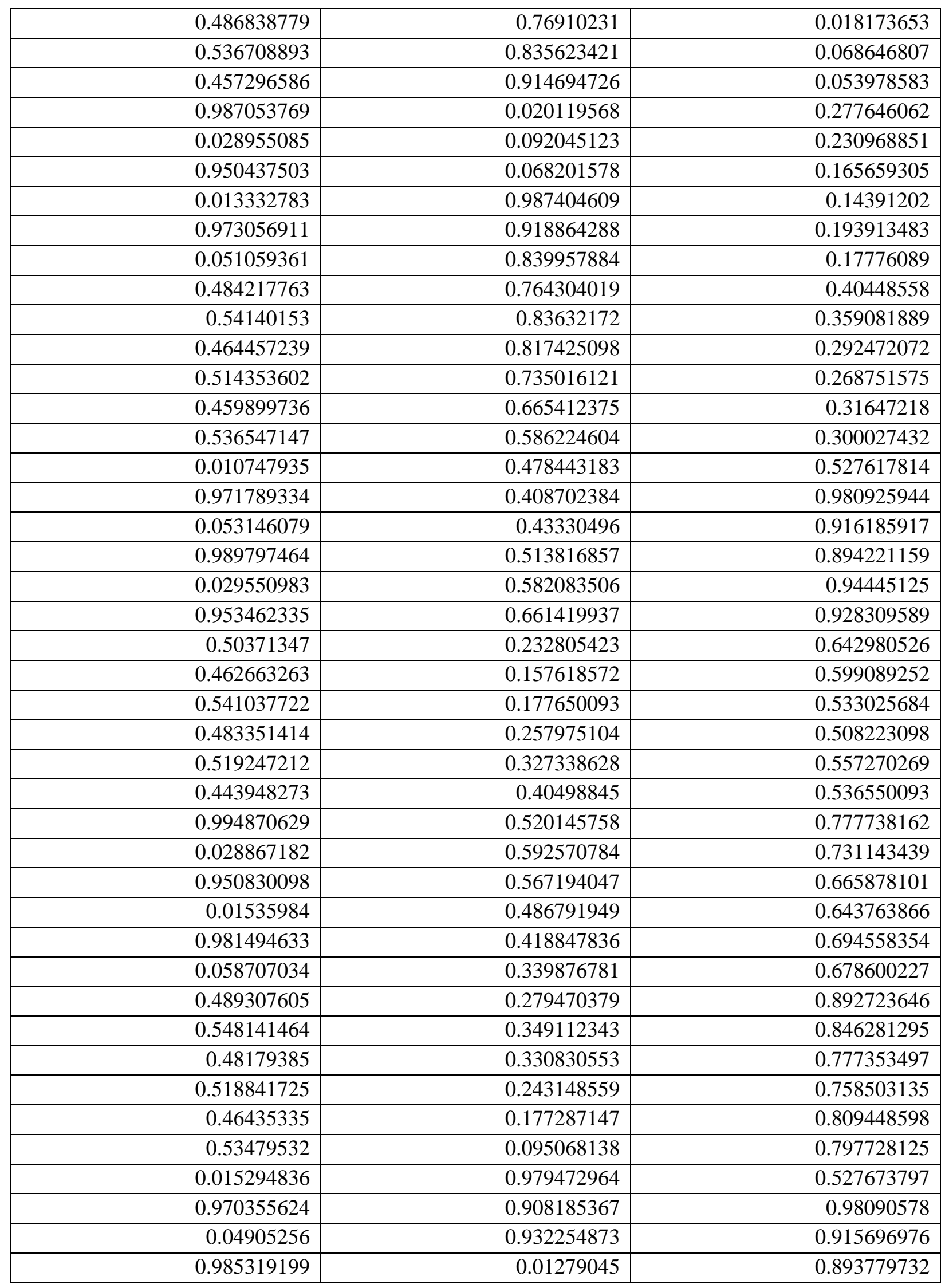




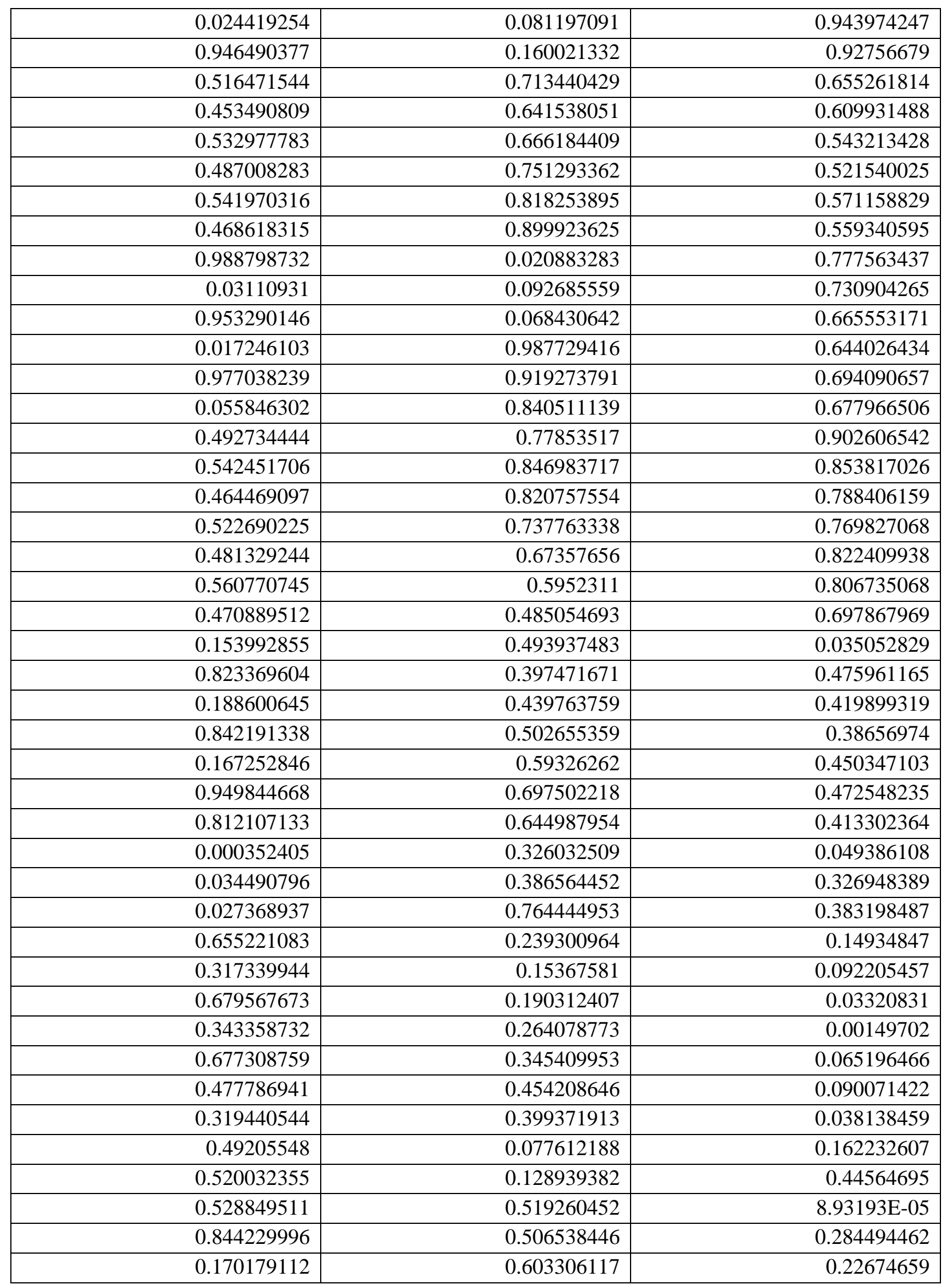




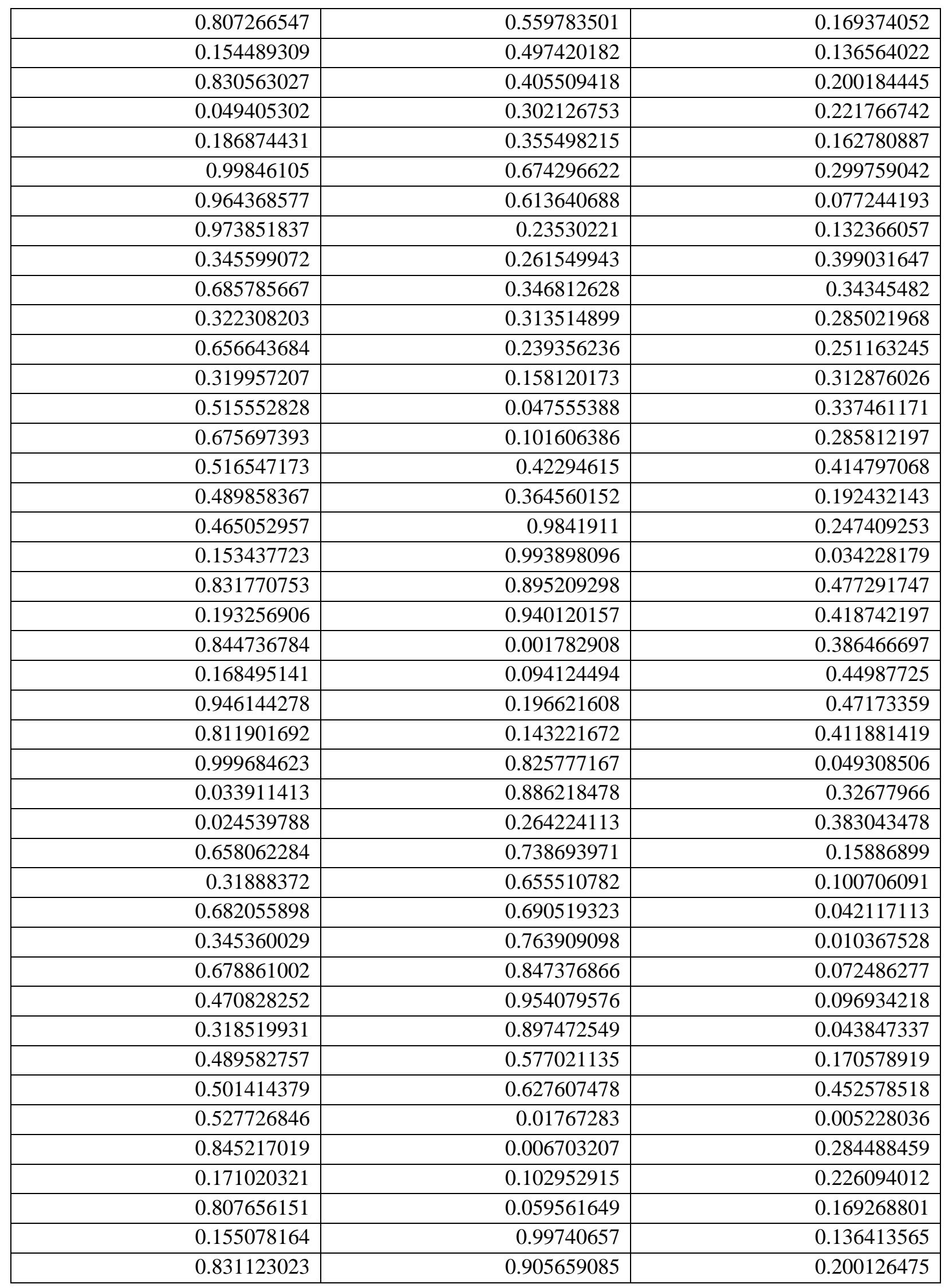




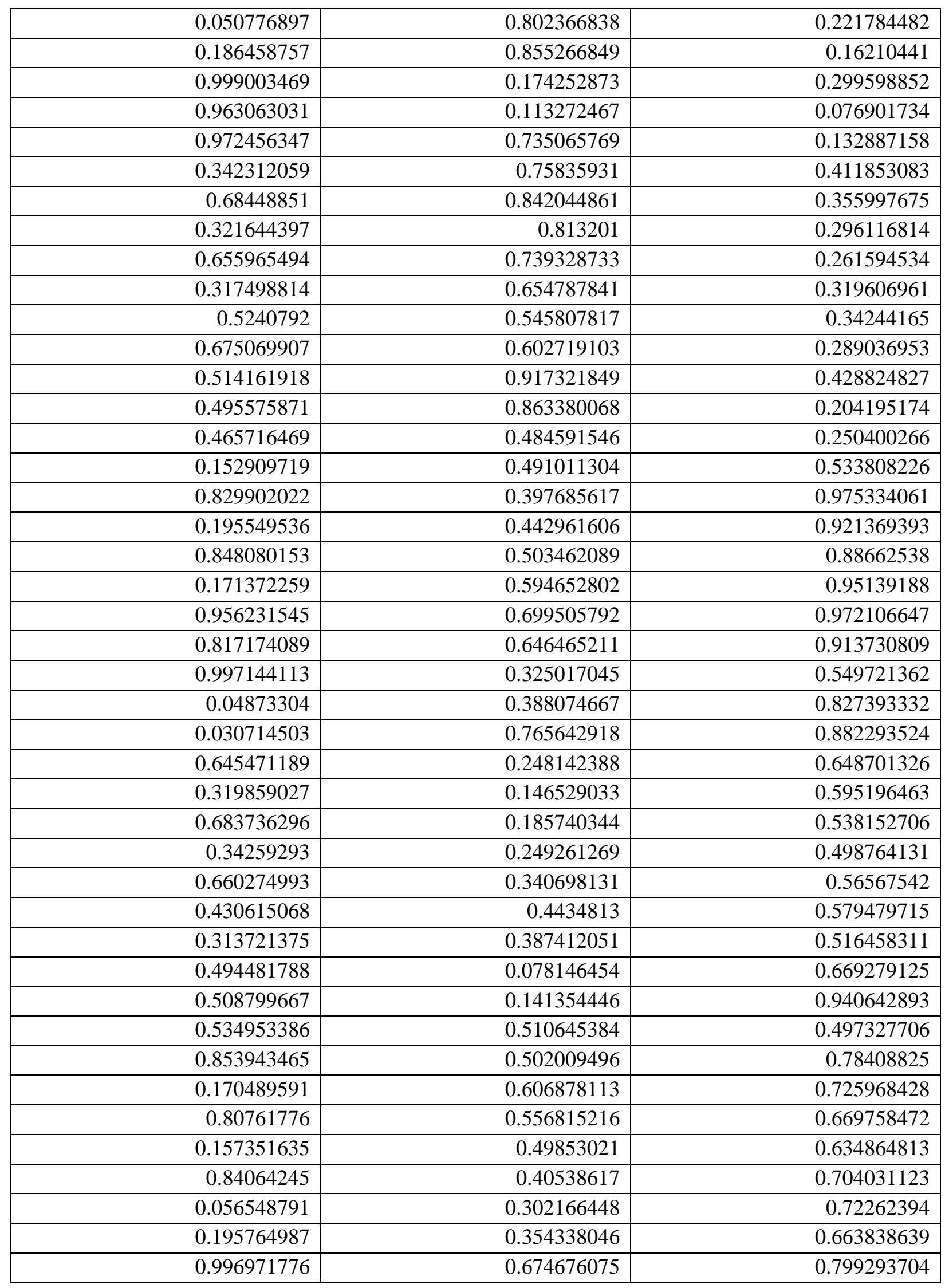




\begin{tabular}{|c|c|c|}
\hline 0.964857637 & 0.611430261 & 0.576620334 \\
\hline 0.979735554 & 0.235444599 & 0.633215404 \\
\hline 0.346691878 & 0.272148034 & 0.898568968 \\
\hline 0.691191865 & 0.358035321 & 0.846748807 \\
\hline 0.338188034 & 0.32854535 & 0.7802316 \\
\hline 0.659097633 & 0.243731917 & 0.749530849 \\
\hline 0.321977742 & 0.168799944 & 0.814524865 \\
\hline 0.518365209 & 0.059107807 & 0.842547258 \\
\hline 0.674198912 & 0.108387943 & 0.786925718 \\
\hline 0.505591827 & 0.432018524 & 0.916110364 \\
\hline 0.46144673 & 0.989716972 & 0.753303174 \\
\hline 0.157417834 & 0.993219559 & 0.534096104 \\
\hline 0.828329916 & 0.896860635 & 0.976317196 \\
\hline 0.191677562 & 0.941494336 & 0.919507913 \\
\hline 0.843640915 & 0.002615313 & 0.885905589 \\
\hline 0.166265216 & 0.094682366 & 0.950278068 \\
\hline 0.944368828 & 0.197174906 & 0.971753901 \\
\hline 0.811884902 & 0.14475029 & 0.910839777 \\
\hline 0.0044761 & 0.825234937 & 0.549256164 \\
\hline 0.035901599 & 0.887563047 & 0.826717474 \\
\hline 0.028472722 & 0.265005894 & 0.88358262 \\
\hline 0.659342391 & 0.719245626 & 0.660214035 \\
\hline 0.311172654 & 0.641064553 & 0.60569296 \\
\hline 0.67553495 & 0.668268778 & 0.54698417 \\
\hline 0.345535573 & 0.747787847 & 0.514346341 \\
\hline 0.684490551 & 0.828046549 & 0.574866158 \\
\hline 0.48834084 & 0.937336809 & 0.603427299 \\
\hline 0.328292914 & 0.886421874 & 0.550177303 \\
\hline 0.514539409 & 0.642901084 & 0.949535523 \\
\hline 0.539369342 & 0.003794871 & 0.511454335 \\
\hline 0.84663546 & 0.007600602 & 0.783924086 \\
\hline 0.173099662 & 0.104312865 & 0.726443812 \\
\hline 0.810539592 & 0.059552775 & 0.668945625 \\
\hline 0.159034908 & 0.997961772 & 0.63657947 \\
\hline 0.834985512 & 0.905697437 & 0.699835516 \\
\hline 0.057091729 & 0.803280477 & 0.72215063 \\
\hline 0.190916411 & 0.856081857 & 0.661859258 \\
\hline 0.001388401 & 0.175011597 & 0.799077688 \\
\hline 0.96526895 & 0.113520683 & 0.576890659 \\
\hline 0.975394555 & 0.735388232 & 0.633494332 \\
\hline 0.350078169 & 0.767733574 & 0.907962736 \\
\hline 0.685518434 & 0.855181822 & 0.850042196 \\
\hline 0.321547603 & 0.813346104 & 0.792941695 \\
\hline
\end{tabular}




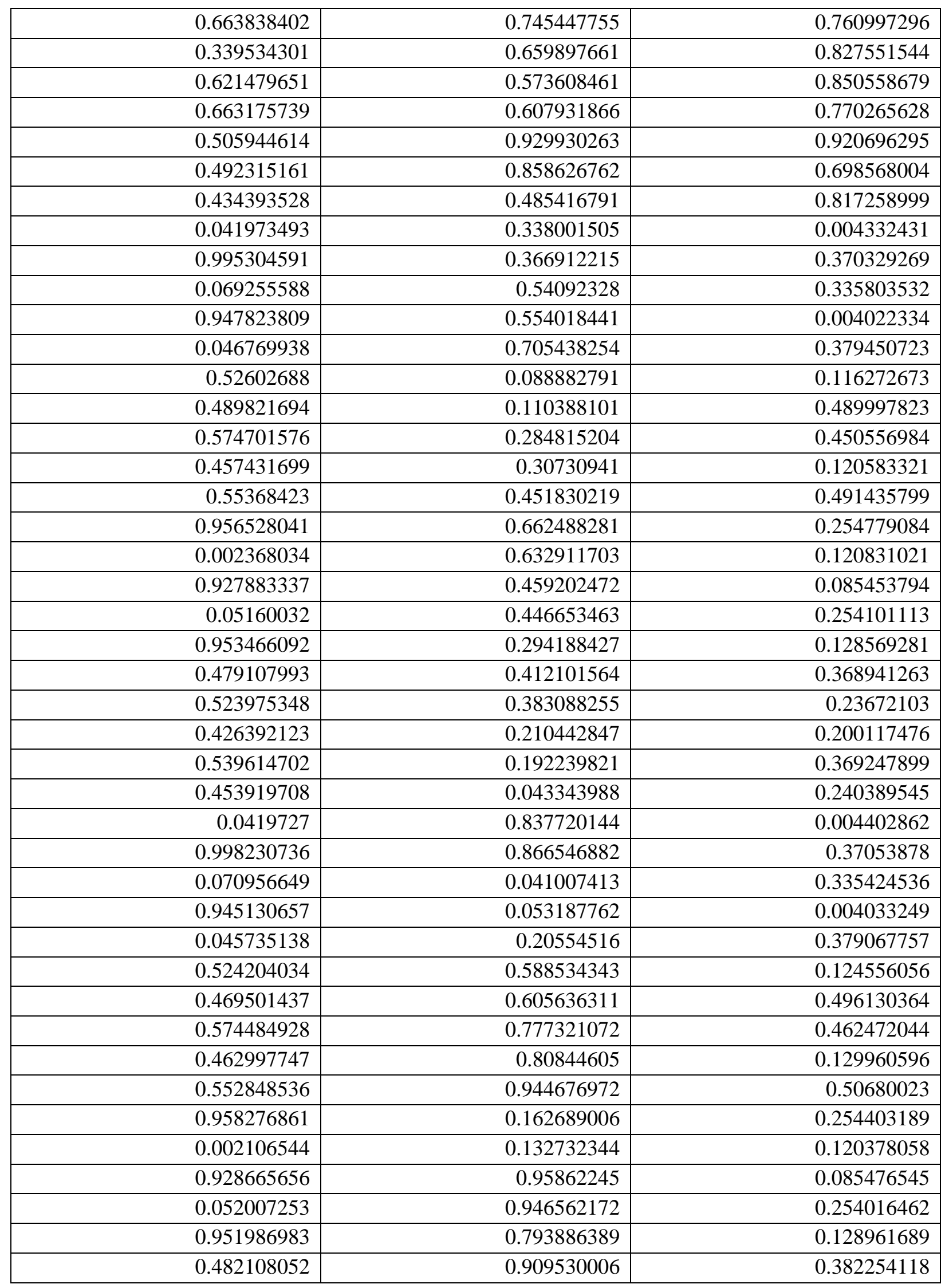




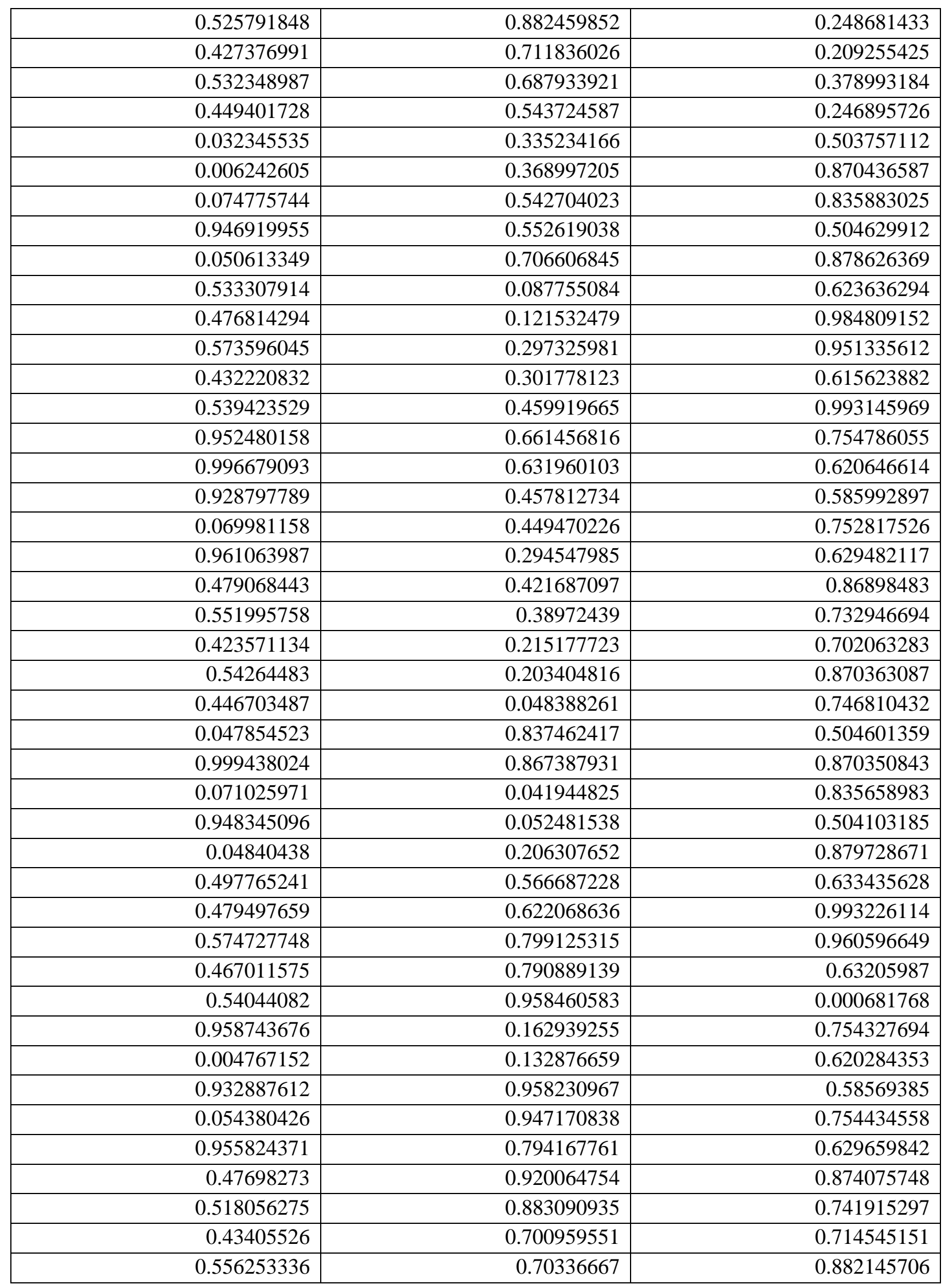


Site 04 co-ordinates:

$\mathrm{a}=7.784 \AA, \mathrm{b}=16.402 \AA, \mathrm{c}=20.760 \AA$

$\Upsilon=96.550$

$\mathrm{C} \mathrm{Mg} \mathrm{H} \mathrm{O}$

$\begin{array}{lll}96 & 1 & 15880\end{array}$

\begin{tabular}{|c|c|c|}
\hline $\mathrm{x}$ & $\mathrm{y}$ & $\mathrm{z}$ \\
\hline 0.013314308 & 0.480978197 & 0.026877515 \\
\hline 0.970864016 & 0.407685872 & 0.479730044 \\
\hline 0.050152398 & 0.431952714 & 0.414684791 \\
\hline 0.986577448 & 0.512742582 & 0.393041406 \\
\hline 0.02697523 & 0.581140246 & 0.443141196 \\
\hline 0.950576621 & 0.660516315 & 0.427047037 \\
\hline 0.515582114 & 0.232269011 & 0.142651828 \\
\hline 0.461247779 & 0.163511882 & 0.095143877 \\
\hline 0.535854414 & 0.186565221 & 0.029223317 \\
\hline 0.486047431 & 0.270450454 & 0.00857904 \\
\hline 0.539442168 & 0.336414439 & 0.059631891 \\
\hline 0.467352784 & 0.417777989 & 0.047006657 \\
\hline 0.987820449 & 0.520300887 & 0.276789825 \\
\hline 0.028616774 & 0.592445607 & 0.230305775 \\
\hline 0.950946898 & 0.568460629 & 0.164902075 \\
\hline 0.015055719 & 0.487871411 & 0.143201624 \\
\hline 0.975086221 & 0.419226708 & 0.193099582 \\
\hline 0.053589903 & 0.340503155 & 0.17674618 \\
\hline 0.487160898 & 0.267093384 & 0.393720658 \\
\hline 0.541545483 & 0.338542585 & 0.348029676 \\
\hline 0.465764505 & 0.318229603 & 0.281819317 \\
\hline 0.516105134 & 0.235412677 & 0.259101822 \\
\hline 0.461289204 & 0.166926359 & 0.307943828 \\
\hline 0.534821258 & 0.086268143 & 0.293885659 \\
\hline 0.013599664 & 0.981265231 & 0.026885286 \\
\hline 0.974886228 & 0.907263555 & 0.48048049 \\
\hline 0.051046637 & 0.931564888 & 0.41486965 \\
\hline 0.986275311 & 0.011992836 & 0.393293947 \\
\hline 0.026434571 & 0.080635014 & 0.443192784 \\
\hline 0.9474345 & 0.159354255 & 0.427098423 \\
\hline 0.516862341 & 0.732186065 & 0.153936458 \\
\hline 0.463118743 & 0.66364034 & 0.105851326 \\
\hline 0.538574174 & 0.686966853 & 0.039973396 \\
\hline
\end{tabular}




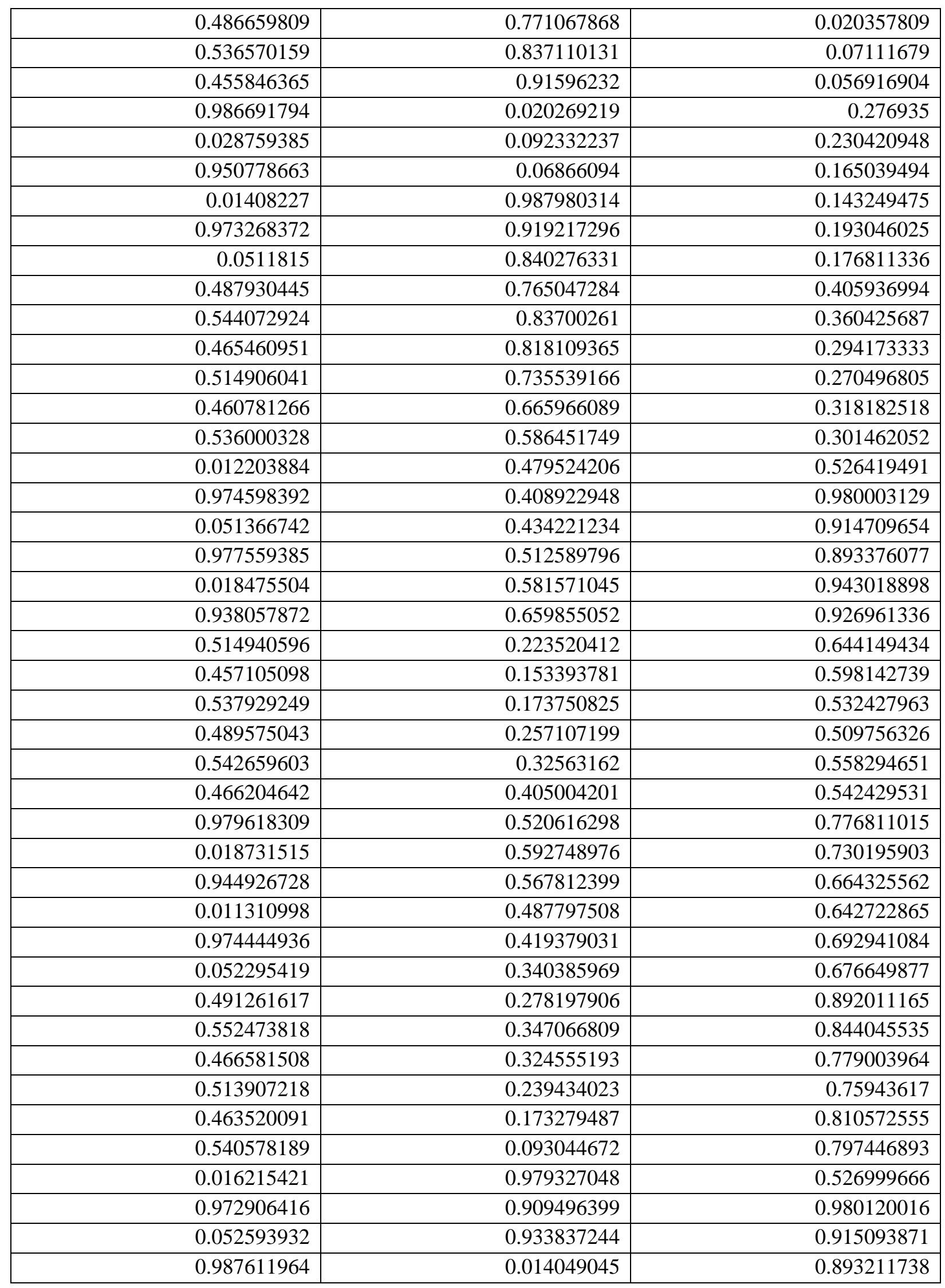




\begin{tabular}{|c|c|c|}
\hline 0.02637454 & 0.082576973 & 0.94328246 \\
\hline 0.94782107 & 0.161189779 & 0.926744653 \\
\hline 0.519290966 & 0.712281661 & 0.657522044 \\
\hline 0.456456554 & 0.642132098 & 0.610055012 \\
\hline 0.537614414 & 0.666769274 & 0.543885108 \\
\hline 0.491521704 & 0.751342667 & 0.522589667 \\
\hline 0.546396115 & 0.81774706 & 0.572537659 \\
\hline 0.469676214 & 0.898334267 & 0.560411869 \\
\hline 0.989920142 & 0.021320789 & 0.77690617 \\
\hline 0.030831747 & 0.092977311 & 0.729945835 \\
\hline 0.952462851 & 0.06827147 & 0.664789624 \\
\hline 0.018370144 & 0.988009735 & 0.643215697 \\
\hline 0.980204288 & 0.919689903 & 0.693582793 \\
\hline 0.060087796 & 0.841164787 & 0.677580022 \\
\hline 0.489488171 & 0.783217915 & 0.905194574 \\
\hline 0.548419534 & 0.848293094 & 0.855004943 \\
\hline 0.47118685 & 0.820775635 & 0.789536487 \\
\hline 0.517468797 & 0.733870334 & 0.77313592 \\
\hline 0.45540277 & 0.675245926 & 0.827884222 \\
\hline 0.503893344 & 0.588282303 & 0.822149145 \\
\hline 0.456656636 & 0.501438832 & 0.705492729 \\
\hline 0.15493959 & 0.495694803 & 0.033590102 \\
\hline 0.829084179 & 0.396355443 & 0.474613277 \\
\hline 0.192867978 & 0.441098342 & 0.418592786 \\
\hline 0.844766087 & 0.50272671 & 0.385596544 \\
\hline 0.168836506 & 0.593833421 & 0.44968264 \\
\hline 0.951088013 & 0.698065857 & 0.471109522 \\
\hline 0.815185706 & 0.646012795 & 0.411125684 \\
\hline 0.005837977 & 0.326574561 & 0.048124698 \\
\hline 0.037805581 & 0.387239657 & 0.32578093 \\
\hline 0.03157065 & 0.765111602 & 0.382147118 \\
\hline 0.657744233 & 0.240117184 & 0.149416098 \\
\hline 0.31800573 & 0.156125776 & 0.092552595 \\
\hline 0.678863732 & 0.191157232 & 0.032256022 \\
\hline 0.344756208 & 0.267084855 & 0.000993569 \\
\hline 0.681653669 & 0.345489477 & 0.064585014 \\
\hline 0.485745052 & 0.455709373 & 0.090779663 \\
\hline 0.327462693 & 0.403439288 & 0.037581192 \\
\hline 0.491674692 & 0.078780833 & 0.161652049 \\
\hline 0.509816499 & 0.127519428 & 0.444334105 \\
\hline 0.536261061 & 0.520779792 & 0.999509845 \\
\hline 0.845966946 & 0.506625488 & 0.283576555 \\
\hline 0.170608643 & 0.604368016 & 0.225842395 \\
\hline
\end{tabular}




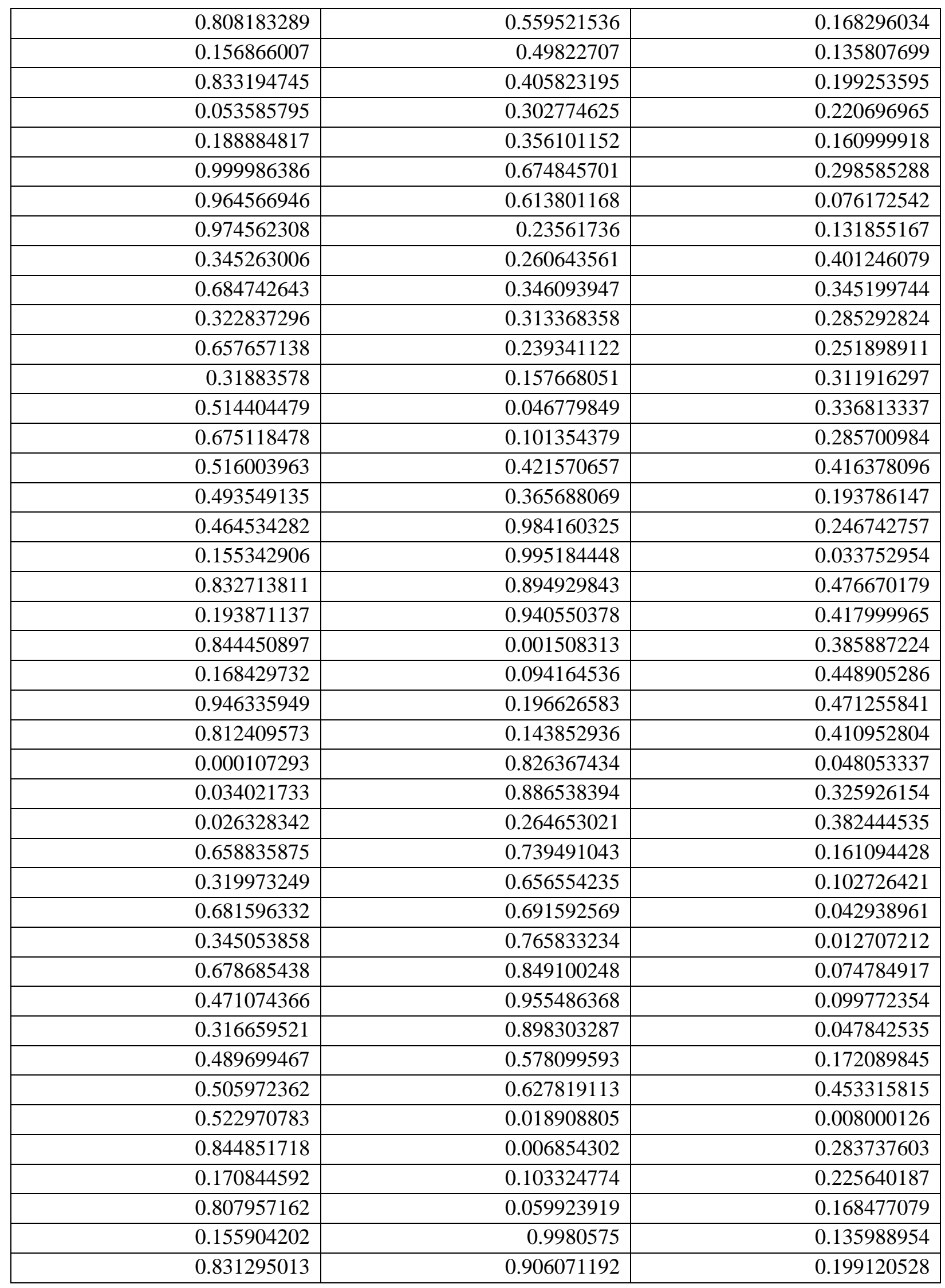




\begin{tabular}{|c|c|c|}
\hline 0.05094973 & 0.802643166 & 0.220823056 \\
\hline 0.18658137 & 0.855569947 & 0.161177936 \\
\hline 0.999609353 & 0.174519563 & 0.298997916 \\
\hline 0.964369093 & 0.113984688 & 0.076347646 \\
\hline 0.972238609 & 0.73548925 & 0.131738993 \\
\hline 0.346192677 & 0.759152449 & 0.41366655 \\
\hline 0.687015449 & 0.842840169 & 0.356611782 \\
\hline 0.322743438 & 0.813981938 & 0.298236394 \\
\hline 0.656550529 & 0.739855198 & 0.263396896 \\
\hline 0.31845878 & 0.655608378 & 0.321750437 \\
\hline 0.524056405 & 0.546310681 & 0.344061083 \\
\hline 0.674350656 & 0.602516423 & 0.289964336 \\
\hline 0.51756662 & 0.917344224 & 0.430686339 \\
\hline 0.496578304 & 0.863561865 & 0.205691873 \\
\hline 0.46459109 & 0.485007615 & 0.25185281 \\
\hline 0.154132836 & 0.492768896 & 0.533380873 \\
\hline 0.832758163 & 0.396532029 & 0.975240142 \\
\hline 0.193900382 & 0.446961117 & 0.918003479 \\
\hline 0.835534799 & 0.499809633 & 0.887312152 \\
\hline 0.160656705 & 0.595544332 & 0.947810228 \\
\hline 0.937845039 & 0.697412137 & 0.970999314 \\
\hline 0.802977405 & 0.643765648 & 0.911348237 \\
\hline 0.998752555 & 0.325494658 & 0.548250944 \\
\hline 0.045284922 & 0.389297148 & 0.826153437 \\
\hline 0.021049192 & 0.765241281 & 0.883114713 \\
\hline 0.656979606 & 0.229628362 & 0.651204286 \\
\hline 0.314208636 & 0.148803697 & 0.594309658 \\
\hline 0.680547272 & 0.177767922 & 0.537292166 \\
\hline 0.348012902 & 0.252995141 & 0.502492736 \\
\hline 0.684899683 & 0.336176266 & 0.562631314 \\
\hline 0.470161879 & 0.443900979 & 0.585917126 \\
\hline 0.329587975 & 0.388833637 & 0.52834009 \\
\hline 0.483309972 & 0.071848355 & 0.668042021 \\
\hline 0.507398144 & 0.140915504 & 0.941060414 \\
\hline 0.541495103 & 0.510035439 & 0.499241402 \\
\hline 0.837747302 & 0.50343201 & 0.782197004 \\
\hline 0.161212497 & 0.605627455 & 0.72745976 \\
\hline 0.801422645 & 0.557529854 & 0.666849211 \\
\hline 0.153682566 & 0.499721592 & 0.634964304 \\
\hline 0.83267648 & 0.405176518 & 0.699295604 \\
\hline 0.055292826 & 0.303368898 & 0.72088288 \\
\hline 0.187328552 & 0.355725938 & 0.659757361 \\
\hline 0.983928848 & 0.675091023 & 0.797466762 \\
\hline
\end{tabular}




\begin{tabular}{|c|c|c|}
\hline 0.960691914 & 0.612660532 & 0.575355989 \\
\hline 0.973252164 & 0.234874504 & 0.632269259 \\
\hline 0.348627487 & 0.272671714 & 0.897605092 \\
\hline 0.695084905 & 0.34878474 & 0.839663608 \\
\hline 0.325259815 & 0.323095327 & 0.784663593 \\
\hline 0.655421549 & 0.243459118 & 0.751980492 \\
\hline 0.321267924 & 0.162735424 & 0.815494855 \\
\hline 0.529933058 & 0.056704702 & 0.842105977 \\
\hline 0.678792558 & 0.109125171 & 0.785732359 \\
\hline 0.530235779 & 0.427882502 & 0.914121028 \\
\hline 0.508176967 & 0.35331239 & 0.685007175 \\
\hline 0.471262695 & 0.986880495 & 0.753311685 \\
\hline 0.158368172 & 0.993016275 & 0.533334089 \\
\hline 0.830873042 & 0.898209504 & 0.975234977 \\
\hline 0.195253623 & 0.943557817 & 0.919305449 \\
\hline 0.845902334 & 0.003311358 & 0.885520083 \\
\hline 0.168124054 & 0.096288923 & 0.949662308 \\
\hline 0.947526026 & 0.199095702 & 0.970577141 \\
\hline 0.812602305 & 0.145540864 & 0.910868066 \\
\hline 0.007984895 & 0.825396969 & 0.548676307 \\
\hline 0.04192066 & 0.888478861 & 0.826334247 \\
\hline 0.029387978 & 0.266073512 & 0.88209208 \\
\hline 0.661609775 & 0.716927245 & 0.663966726 \\
\hline 0.314422668 & 0.642803804 & 0.605444127 \\
\hline 0.68013534 & 0.669152685 & 0.547861096 \\
\hline 0.350015654 & 0.747901352 & 0.515465525 \\
\hline 0.688935549 & 0.828332999 & 0.575683232 \\
\hline 0.484104509 & 0.935073511 & 0.604864073 \\
\hline 0.330461752 & 0.883429464 & 0.549695476 \\
\hline 0.504347617 & 0.64481875 & 0.951247202 \\
\hline 0.5350958 & 0.002844024 & 0.513231104 \\
\hline 0.848042458 & 0.007061516 & 0.783446592 \\
\hline 0.172841436 & 0.104060141 & 0.725162311 \\
\hline 0.809733438 & 0.058358184 & 0.668476712 \\
\hline 0.160054917 & 0.999050092 & 0.63555024 \\
\hline 0.838496083 & 0.905452386 & 0.699744393 \\
\hline 0.059670409 & 0.803534592 & 0.721601086 \\
\hline 0.195774138 & 0.856825195 & 0.662176501 \\
\hline 0.999851338 & 0.175275207 & 0.798382231 \\
\hline 0.966350596 & 0.112655685 & 0.575604704 \\
\hline 0.977141826 & 0.73618133 & 0.633192042 \\
\hline 0.346657734 & 0.777761837 & 0.910896958 \\
\hline 0.691323173 & 0.852969625 & 0.851806548 \\
\hline
\end{tabular}




\begin{tabular}{|c|c|c|}
\hline 0.328313822 & 0.817347288 & 0.793018696 \\
\hline 0.659046207 & 0.734850131 & 0.767483227 \\
\hline 0.313687385 & 0.673127934 & 0.833278556 \\
\hline 0.500023606 & 0.562809683 & 0.871885834 \\
\hline 0.643394278 & 0.59366457 & 0.807775241 \\
\hline 0.512020671 & 0.933021095 & 0.920209963 \\
\hline 0.50540238 & 0.856777972 & 0.699374428 \\
\hline 0.048435461 & 0.338996137 & 0.003324829 \\
\hline$-4.23505 \mathrm{E}-06$ & 0.367458459 & 0.369351149 \\
\hline 0.071136246 & 0.541526854 & 0.334705871 \\
\hline 0.944469201 & 0.553673307 & 0.003897642 \\
\hline 0.051335283 & 0.706147172 & 0.378484547 \\
\hline 0.525815783 & 0.090393032 & 0.115805093 \\
\hline 0.478038365 & 0.109103778 & 0.488688566 \\
\hline 0.577497905 & 0.282719729 & 0.451339829 \\
\hline 0.461726034 & 0.309036316 & 0.120449107 \\
\hline 0.561048053 & 0.451153152 & 0.493170241 \\
\hline 0.955589311 & 0.662391076 & 0.254048778 \\
\hline 0.002332179 & 0.633246121 & 0.119824769 \\
\hline 0.930970611 & 0.458648761 & 0.084775901 \\
\hline 0.053759206 & 0.447007067 & 0.253304954 \\
\hline 0.954691912 & 0.294520861 & 0.127887202 \\
\hline 0.476963531 & 0.410638769 & 0.370829024 \\
\hline 0.526300369 & 0.383446271 & 0.238369925 \\
\hline 0.428064477 & 0.21195328 & 0.200071262 \\
\hline 0.536702323 & 0.190798288 & 0.369679348 \\
\hline 0.453990408 & 0.043408967 & 0.239679568 \\
\hline 0.044007556 & 0.838908939 & 0.003465992 \\
\hline 0.999350027 & 0.86674902 & 0.369777137 \\
\hline 0.07043928 & 0.041214013 & 0.334800265 \\
\hline 0.947106237 & 0.054494861 & 0.00332641 \\
\hline 0.047247514 & 0.205880507 & 0.378790514 \\
\hline 0.526058413 & 0.590031961 & 0.126404278 \\
\hline 0.475386304 & 0.605584209 & 0.496913431 \\
\hline 0.578834161 & 0.77824673 & 0.463693436 \\
\hline 0.46387213 & 0.80960032 & 0.132543039 \\
\hline 0.555501177 & 0.944672182 & 0.508907955 \\
\hline 0.957893918 & 0.162895961 & 0.253996096 \\
\hline 0.002821776 & 0.133279894 & 0.119937694 \\
\hline 0.930039659 & 0.959624706 & 0.084643818 \\
\hline 0.051748825 & 0.94680298 & 0.253333404 \\
\hline 0.951952728 & 0.794387009 & 0.128006268 \\
\hline 0.485519528 & 0.910208665 & 0.384084334 \\
\hline
\end{tabular}




\begin{tabular}{|c|c|c|}
\hline 0.526244846 & 0.883024948 & 0.250221138 \\
\hline 0.427968954 & 0.712299098 & 0.211186451 \\
\hline 0.535176431 & 0.688528654 & 0.380416353 \\
\hline 0.446950167 & 0.543971054 & 0.248760187 \\
\hline 0.041632018 & 0.337071795 & 0.50349167 \\
\hline 0.00735483 & 0.368751408 & 0.869379404 \\
\hline 0.056415153 & 0.543465288 & 0.834728902 \\
\hline 0.947141698 & 0.552986038 & 0.503135321 \\
\hline 0.036133524 & 0.706075823 & 0.878017 \\
\hline 0.51348393 & 0.079572237 & 0.621188718 \\
\hline 0.473356713 & 0.122558839 & 0.98531629 \\
\hline 0.575730586 & 0.297019104 & 0.950131342 \\
\hline 0.467389649 & 0.300976726 & 0.620048408 \\
\hline 0.552145835 & 0.461752797 & 0.994204901 \\
\hline 0.941403385 & 0.661896584 & 0.752744579 \\
\hline 0.993772178 & 0.632581086 & 0.619334855 \\
\hline 0.928479112 & 0.458071259 & 0.58434897 \\
\hline 0.05380493 & 0.448360601 & 0.752414651 \\
\hline 0.951494233 & 0.293735195 & 0.628920071 \\
\hline 0.502663413 & 0.421245798 & 0.867212657 \\
\hline 0.520687112 & 0.384238063 & 0.727348726 \\
\hline 0.425679695 & 0.20977392 & 0.701892493 \\
\hline 0.54124396 & 0.200497306 & 0.870893142 \\
\hline 0.453172914 & 0.045391772 & 0.746919663 \\
\hline 0.04963831 & 0.837645084 & 0.5037326 \\
\hline 0.004059904 & 0.86900701 & 0.86989356 \\
\hline 0.072203552 & 0.043349618 & 0.83479586 \\
\hline 0.949323649 & 0.052669439 & 0.503616666 \\
\hline 0.047332869 & 0.206985577 & 0.877887617 \\
\hline 0.497943302 & 0.565326417 & 0.630520212 \\
\hline 0.476030489 & 0.623429548 & 0.995529237 \\
\hline 0.574149456 & 0.802614322 & 0.963128902 \\
\hline 0.473498353 & 0.790671985 & 0.633721892 \\
\hline 0.535255653 & 0.959804163 & 0.002994539 \\
\hline 0.960145478 & 0.163934391 & 0.753026963 \\
\hline 0.00118778 & 0.133151421 & 0.619329475 \\
\hline 0.933970472 & 0.958253416 & 0.585031575 \\
\hline 0.058053598 & 0.948484071 & 0.753633904 \\
\hline 0.961627159 & 0.795355817 & 0.628633871 \\
\hline 0.491981835 & 0.924763638 & 0.872949675 \\
\hline 0.533000395 & 0.881530294 & 0.742556261 \\
\hline 0.432091672 & 0.700158733 & 0.71575178 \\
\hline 0.540180546 & 0.706213807 & 0.885855974 \\
\hline
\end{tabular}


Site 05 co-ordinates:

$\mathrm{a}=7.784 \AA, \mathrm{b}=16.402 \AA, \mathrm{c}=20.760 \AA$

$\Upsilon=96.550$

$\mathrm{C} \mathrm{Mg} \mathrm{H} \mathrm{O}$

$\begin{array}{lll}96 & 1 & 15880\end{array}$

\begin{tabular}{|c|c|c|}
\hline $\mathrm{x}$ & $\mathrm{y}$ & $\mathrm{z}$ \\
\hline 0.013993337 & 0.47998736 & 0.026070418 \\
\hline 0.953485905 & 0.406478749 & 0.479807752 \\
\hline 0.037389648 & 0.43050625 & 0.415189638 \\
\hline 0.980675256 & 0.512392905 & 0.392806389 \\
\hline 0.021420198 & 0.58005367 & 0.443510771 \\
\hline 0.947669809 & 0.660169127 & 0.427780992 \\
\hline 0.516521485 & 0.231315942 & 0.148653157 \\
\hline 0.461496188 & 0.160850675 & 0.102215112 \\
\hline 0.537134416 & 0.181973504 & 0.035886743 \\
\hline 0.485515762 & 0.264826848 & 0.013558872 \\
\hline 0.538299202 & 0.332902775 & 0.062847978 \\
\hline 0.46042236 & 0.412410504 & 0.048334212 \\
\hline 0.988089189 & 0.519749494 & 0.276515057 \\
\hline 0.031042163 & 0.591800437 & 0.230126546 \\
\hline 0.952407801 & 0.568024941 & 0.164744961 \\
\hline 0.015211398 & 0.487358192 & 0.142587082 \\
\hline 0.974492461 & 0.418676301 & 0.192511038 \\
\hline 0.051700264 & 0.339535321 & 0.176403808 \\
\hline 0.481913843 & 0.271046282 & 0.399975618 \\
\hline 0.539402734 & 0.341677641 & 0.35404095 \\
\hline 0.462801892 & 0.321515779 & 0.287831201 \\
\hline 0.514435988 & 0.238516565 & 0.265431975 \\
\hline 0.463109358 & 0.170167377 & 0.314682809 \\
\hline 0.539311251 & 0.090331179 & 0.299974133 \\
\hline 0.012674697 & 0.979853934 & 0.027572193 \\
\hline 0.970045725 & 0.90773981 & 0.480775025 \\
\hline 0.049308117 & 0.931655223 & 0.415567678 \\
\hline 0.986193995 & 0.012348582 & 0.3937844 \\
\hline 0.026354599 & 0.080870055 & 0.443762081 \\
\hline 0.947827408 & 0.159567788 & 0.427543748 \\
\hline 0.513151108 & 0.729349117 & 0.149901336 \\
\hline 0.459885723 & 0.659437806 & 0.102798365 \\
\hline 0.536155419 & 0.682333647 & 0.037032492 \\
\hline
\end{tabular}




\begin{tabular}{|c|c|c|}
\hline 0.483161229 & 0.765834366 & 0.016676396 \\
\hline 0.533881227 & 0.832648354 & 0.066887668 \\
\hline 0.458190909 & 0.912684117 & 0.052644334 \\
\hline 0.987659907 & 0.020345185 & 0.277502413 \\
\hline 0.029479491 & 0.092243552 & 0.230831467 \\
\hline 0.951219841 & 0.068231239 & 0.165529924 \\
\hline 0.01397856 & 0.98735113 & 0.143856552 \\
\hline 0.973283933 & 0.918982029 & 0.193961949 \\
\hline 0.050555891 & 0.839817218 & 0.178060008 \\
\hline 0.484182362 & 0.772557564 & 0.399679007 \\
\hline 0.538310858 & 0.842922759 & 0.352895161 \\
\hline 0.46248819 & 0.820920393 & 0.286937072 \\
\hline 0.514503265 & 0.737747895 & 0.26576391 \\
\hline 0.463727548 & 0.670450463 & 0.315666999 \\
\hline 0.540151194 & 0.59065029 & 0.301235719 \\
\hline 0.998913178 & 0.477684538 & 0.526666522 \\
\hline 0.97071309 & 0.40869976 & 0.979015179 \\
\hline 0.051916505 & 0.433087973 & 0.914171421 \\
\hline 0.993524343 & 0.514681342 & 0.891927717 \\
\hline 0.032622523 & 0.582248806 & 0.942940261 \\
\hline 0.956376991 & 0.661992804 & 0.928587631 \\
\hline 0.510327435 & 0.216444777 & 0.64864563 \\
\hline 0.461963752 & 0.147399841 & 0.601129679 \\
\hline 0.535842898 & 0.174444692 & 0.535362171 \\
\hline 0.477561745 & 0.25770479 & 0.516382806 \\
\hline 0.519563553 & 0.32280395 & 0.568526172 \\
\hline 0.437429356 & 0.403707331 & 0.556437954 \\
\hline 0.00458336 & 0.522369283 & 0.775589028 \\
\hline 0.044919284 & 0.593564304 & 0.728451817 \\
\hline 0.956762521 & 0.568729837 & 0.664800818 \\
\hline 0.009627737 & 0.486477916 & 0.641856457 \\
\hline 0.976747422 & 0.419579337 & 0.693464429 \\
\hline 0.04490619 & 0.338431611 & 0.677149378 \\
\hline 0.485603042 & 0.269046837 & 0.896978186 \\
\hline 0.5310877 & 0.338552987 & 0.847855635 \\
\hline 0.450430115 & 0.310860975 & 0.783342594 \\
\hline 0.512914144 & 0.229047649 & 0.764297566 \\
\hline 0.467644438 & 0.163758239 & 0.815803498 \\
\hline 0.545353943 & 0.084659489 & 0.801360435 \\
\hline 0.012469788 & 0.979591446 & 0.527466923 \\
\hline 0.971422012 & 0.907934069 & 0.98092719 \\
\hline 0.049898879 & 0.932063818 & 0.91566172 \\
\hline 0.986586409 & 0.012724396 & 0.893890041 \\
\hline
\end{tabular}




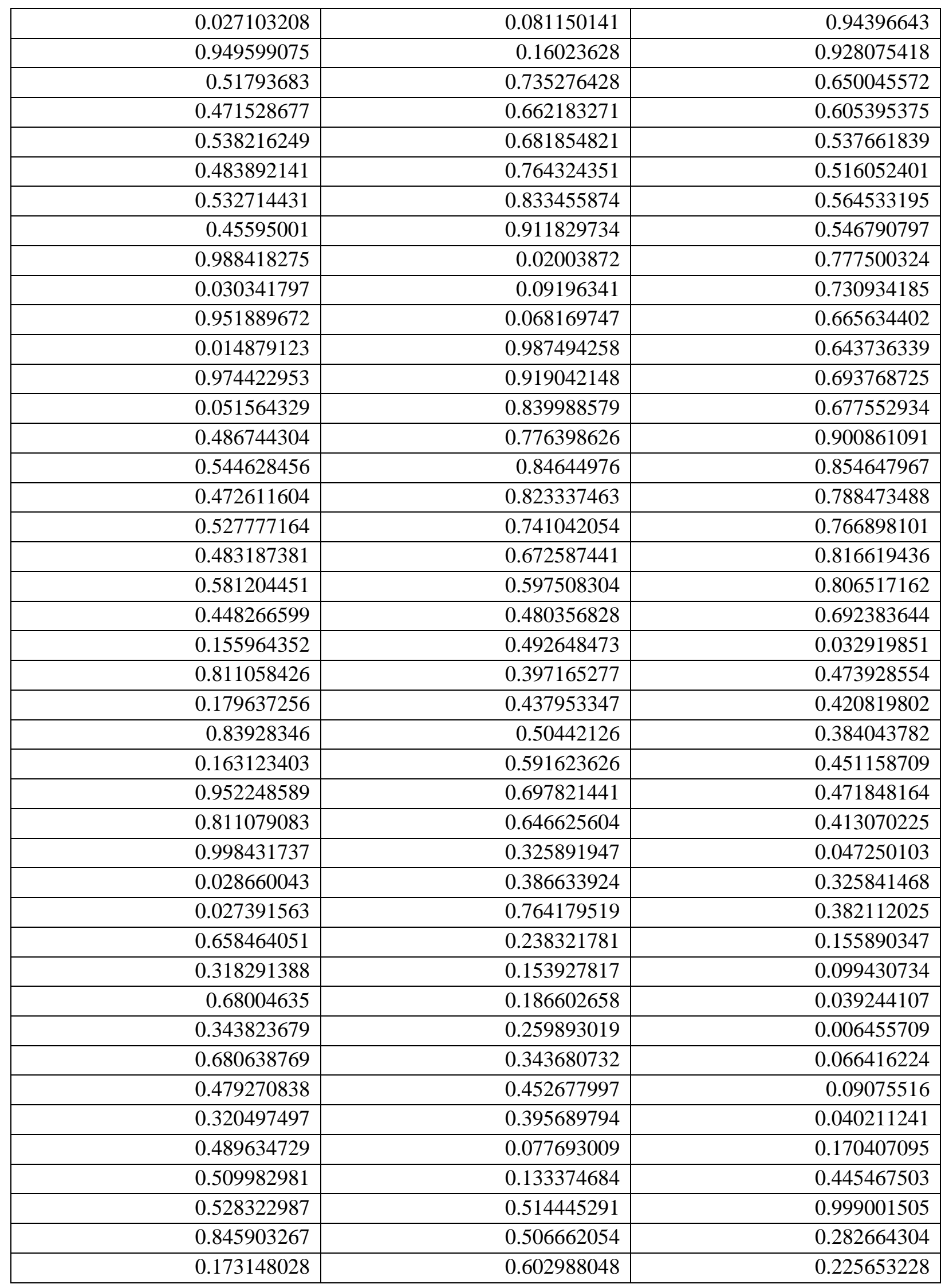




\begin{tabular}{|c|c|c|}
\hline 0.809674869 & 0.559258034 & 0.168548995 \\
\hline 0.156951927 & 0.497222728 & 0.134979586 \\
\hline 0.832481888 & 0.405744876 & 0.198569791 \\
\hline 0.050314694 & 0.301833611 & 0.220412175 \\
\hline 0.187446648 & 0.354457751 & 0.16096421 \\
\hline 0.000781824 & 0.673812602 & 0.298822241 \\
\hline 0.965325926 & 0.613393638 & 0.076019049 \\
\hline 0.972909318 & 0.234926156 & 0.131233038 \\
\hline 0.339300873 & 0.262296934 & 0.405358762 \\
\hline 0.682347884 & 0.347963077 & 0.351290546 \\
\hline 0.319891275 & 0.316836533 & 0.291230059 \\
\hline 0.656106167 & 0.243604371 & 0.258240786 \\
\hline 0.320914537 & 0.159349176 & 0.319229178 \\
\hline 0.52707423 & 0.051540534 & 0.343397727 \\
\hline 0.677772197 & 0.106186765 & 0.288557127 \\
\hline 0.514439925 & 0.421070128 & 0.422719957 \\
\hline 0.493133077 & 0.366772397 & 0.199279724 \\
\hline 0.466241391 & 0.987035686 & 0.252793577 \\
\hline 0.154530058 & 0.993368401 & 0.034379955 \\
\hline 0.828036157 & 0.89687316 & 0.475999924 \\
\hline 0.192028588 & 0.940438195 & 0.419288948 \\
\hline 0.844422147 & 0.002312826 & 0.386212317 \\
\hline 0.168344621 & 0.094104866 & 0.449821483 \\
\hline 0.94661324 & 0.196992782 & 0.471568551 \\
\hline 0.812895779 & 0.14386916 & 0.411425998 \\
\hline 0.000859002 & 0.825700595 & 0.04937088 \\
\hline 0.036234901 & 0.886752873 & 0.32674492 \\
\hline 0.022918928 & 0.264603609 & 0.382371625 \\
\hline 0.655492861 & 0.737725668 & 0.156291264 \\
\hline 0.316758639 & 0.652010573 & 0.099674694 \\
\hline 0.679123874 & 0.687842885 & 0.040558482 \\
\hline 0.341546802 & 0.760730663 & 0.00918692 \\
\hline 0.67613904 & 0.843377715 & 0.071310266 \\
\hline 0.469324496 & 0.950737223 & 0.096465982 \\
\hline 0.32002556 & 0.896909235 & 0.040776809 \\
\hline 0.490896061 & 0.576918019 & 0.170892032 \\
\hline 0.5069327 & 0.634483443 & 0.451115468 \\
\hline 0.531459015 & 0.016474468 & 0.006020206 \\
\hline 0.845711579 & 0.006941236 & 0.284235523 \\
\hline 0.171553918 & 0.10348283 & 0.226231834 \\
\hline 0.808460108 & 0.059632692 & 0.169043743 \\
\hline 0.155792043 & 0.997178524 & 0.136381368 \\
\hline 0.831338669 & 0.906120887 & 0.200315197 \\
\hline
\end{tabular}




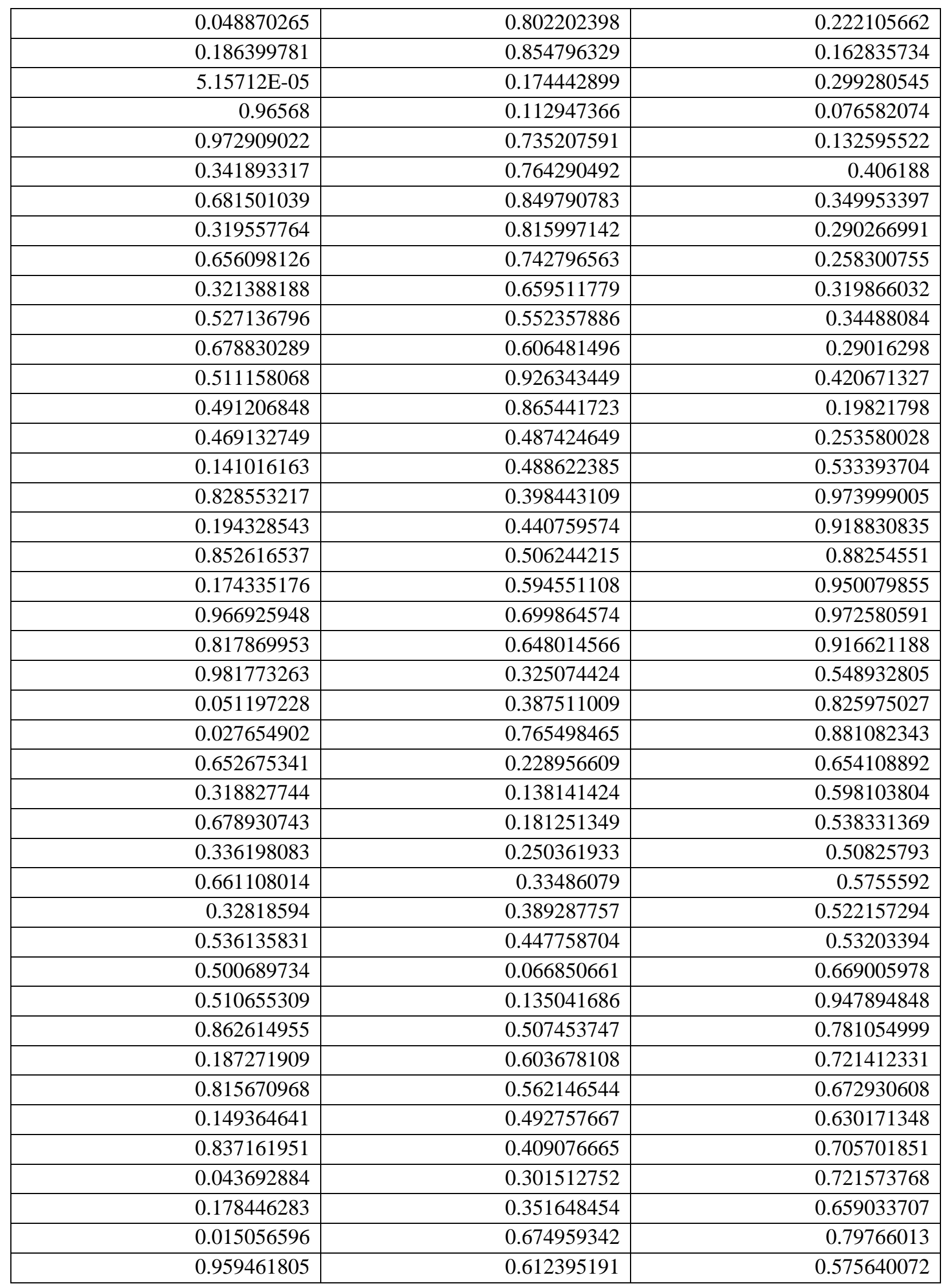




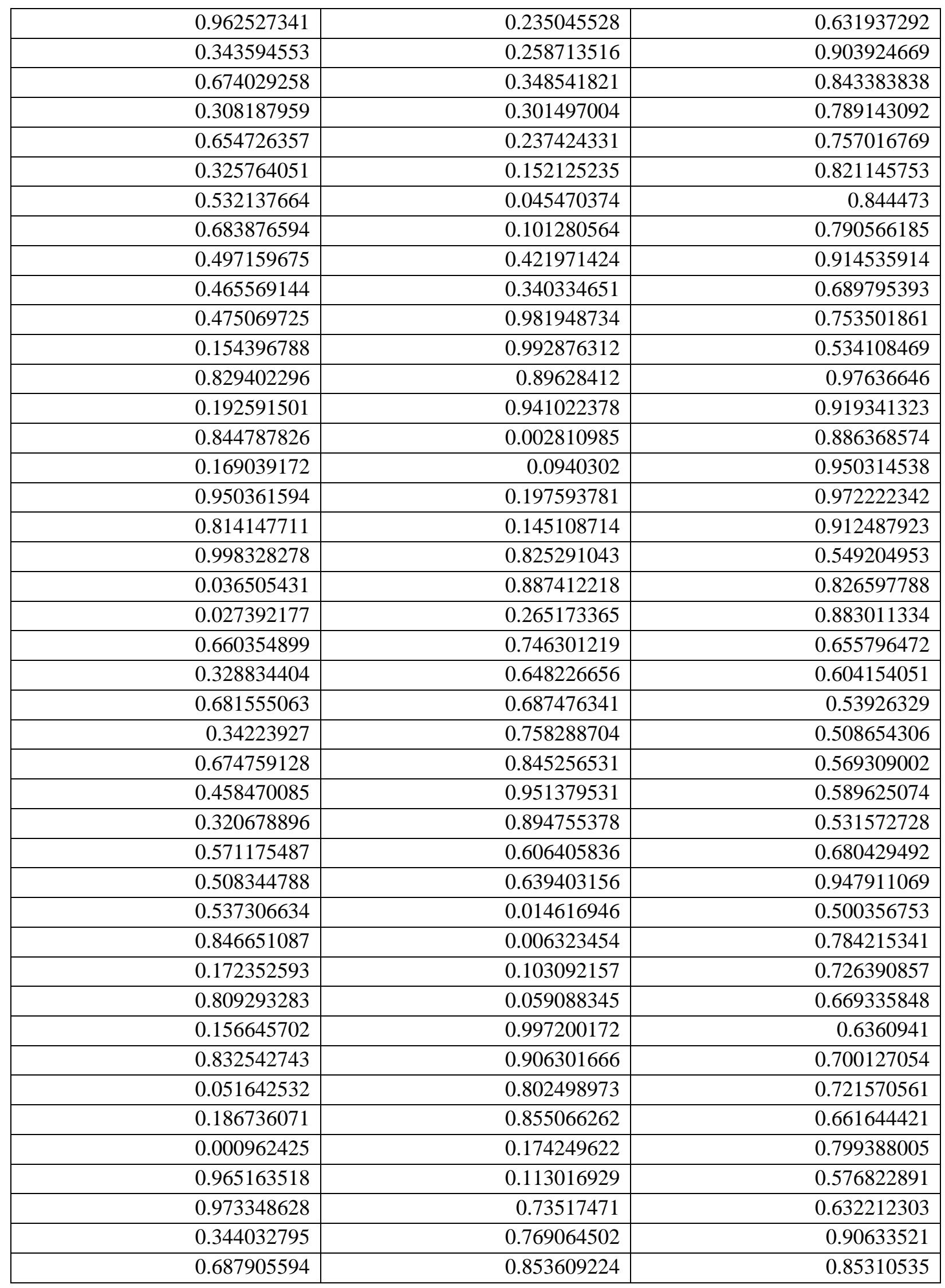




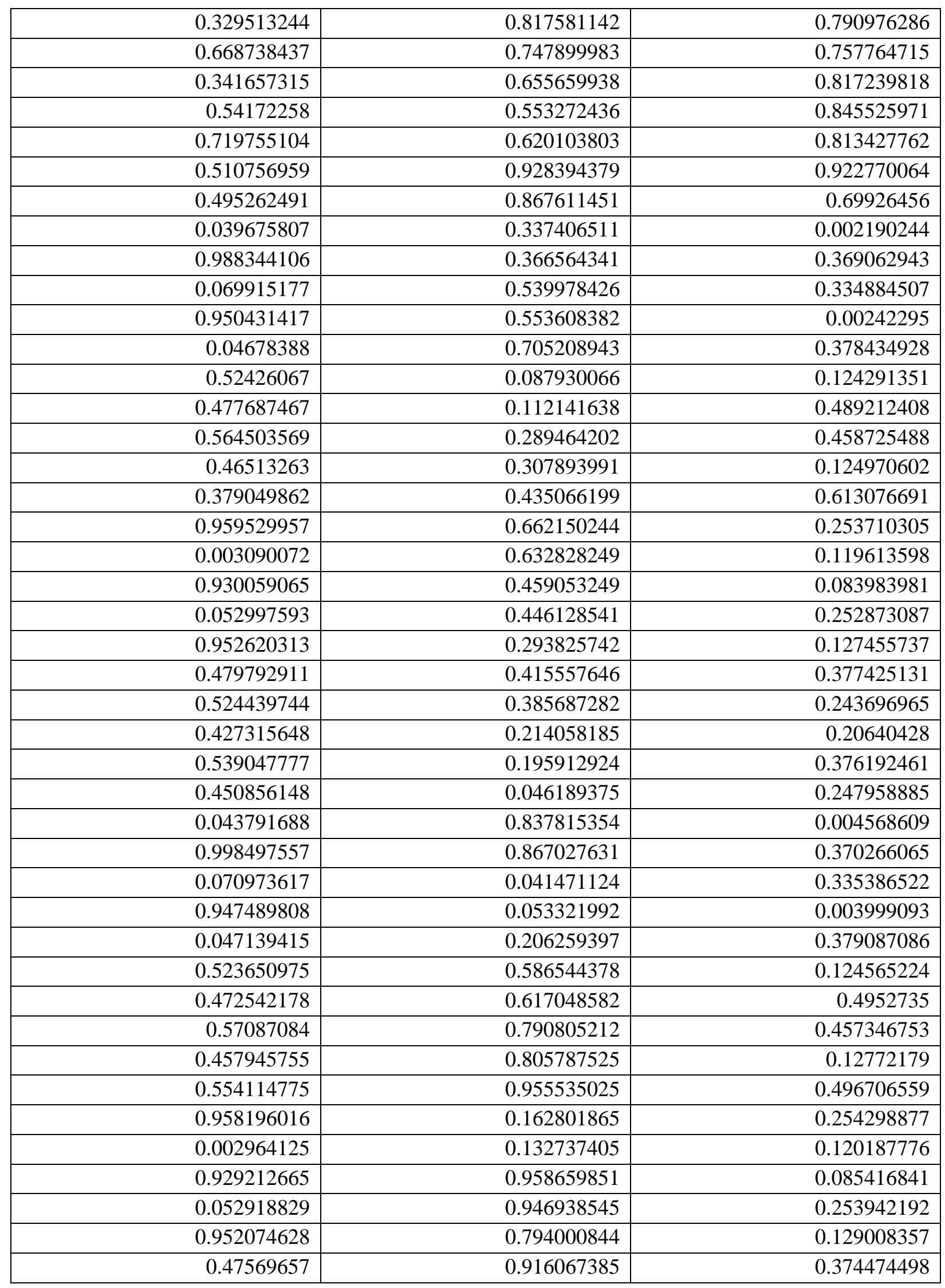




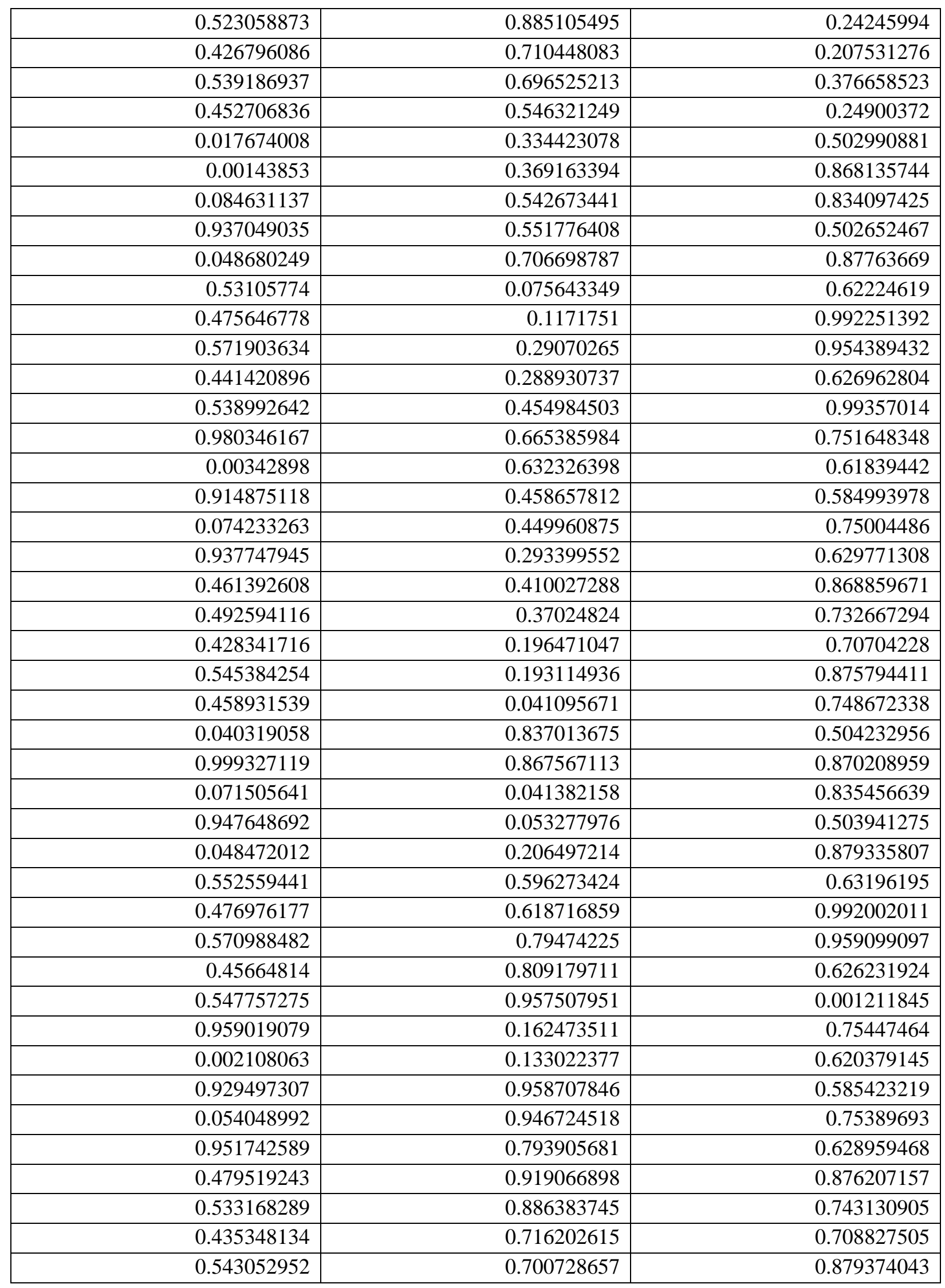




\begin{tabular}{|r|r|r|}
\hline 0.555731024 & 0.56135151 & 0.746471208 \\
\hline
\end{tabular}

Mg-Cell-Mg chair conformer co-ordinates:

$\mathrm{a}=16.352 \AA, \mathrm{b}=16.402 \AA, \mathrm{c}=20.760 \AA$

$\Upsilon=96.550$

C $\mathrm{H} \quad \mathrm{Mg} \quad \mathrm{O}$

$\begin{array}{llll}132 & 220 & 2 & 112\end{array}$

\begin{tabular}{|c|c|c|}
\hline $\mathrm{x}$ & $\mathrm{y}$ & $\mathrm{z}$ \\
\hline 0.272526473 & 0.457212134 & 0.031377108 \\
\hline 0.252542433 & 0.382714773 & 0.488201717 \\
\hline 0.299855841 & 0.409222427 & 0.427173854 \\
\hline 0.266048923 & 0.48552281 & 0.399717622 \\
\hline 0.275988436 & 0.556090591 & 0.449079492 \\
\hline 0.237111369 & 0.633204633 & 0.430229124 \\
\hline 0.268542244 & 0.493392446 & 0.283199124 \\
\hline 0.278189453 & 0.568089598 & 0.238193995 \\
\hline 0.24278284 & 0.543459027 & 0.171943337 \\
\hline 0.275183531 & 0.465152064 & 0.147105947 \\
\hline 0.263603647 & 0.395276006 & 0.195994304 \\
\hline 0.297780394 & 0.316245991 & 0.175204059 \\
\hline 0.482618071 & 0.254458984 & 0.39830823 \\
\hline 0.494773409 & 0.316654442 & 0.343241109 \\
\hline 0.469735558 & 0.273441876 & 0.280089844 \\
\hline 0.512825525 & 0.199582854 & 0.264803248 \\
\hline 0.490498207 & 0.140507035 & 0.321348107 \\
\hline 0.53087383 & 0.061830752 & 0.315893121 \\
\hline 0.275433132 & 0.95624505 & 0.034781993 \\
\hline 0.251886181 & 0.883233413 & 0.488262884 \\
\hline 0.289264038 & 0.907464903 & 0.422882029 \\
\hline 0.259937337 & 0.988725996 & 0.401143924 \\
\hline 0.277121334 & 0.056959485 & 0.451607774 \\
\hline 0.237861699 & 0.135035996 & 0.435758308 \\
\hline 0.262982458 & 0.998636191 & 0.284962401 \\
\hline 0.284087375 & 0.070379206 & 0.238333331 \\
\hline 0.247906855 & 0.045537494 & 0.17269125 \\
\hline 0.27758229 & 0.964342871 & 0.151215526 \\
\hline 0.257992816 & 0.896306331 & 0.201571878 \\
\hline 0.29485596 & 0.816702593 & 0.186493442 \\
\hline 0.497893601 & 0.750678821 & 0.415663296 \\
\hline 0.523657076 & 0.821940181 & 0.369491299 \\
\hline 0.486519664 & 0.799351735 & 0.303662411 \\
\hline
\end{tabular}




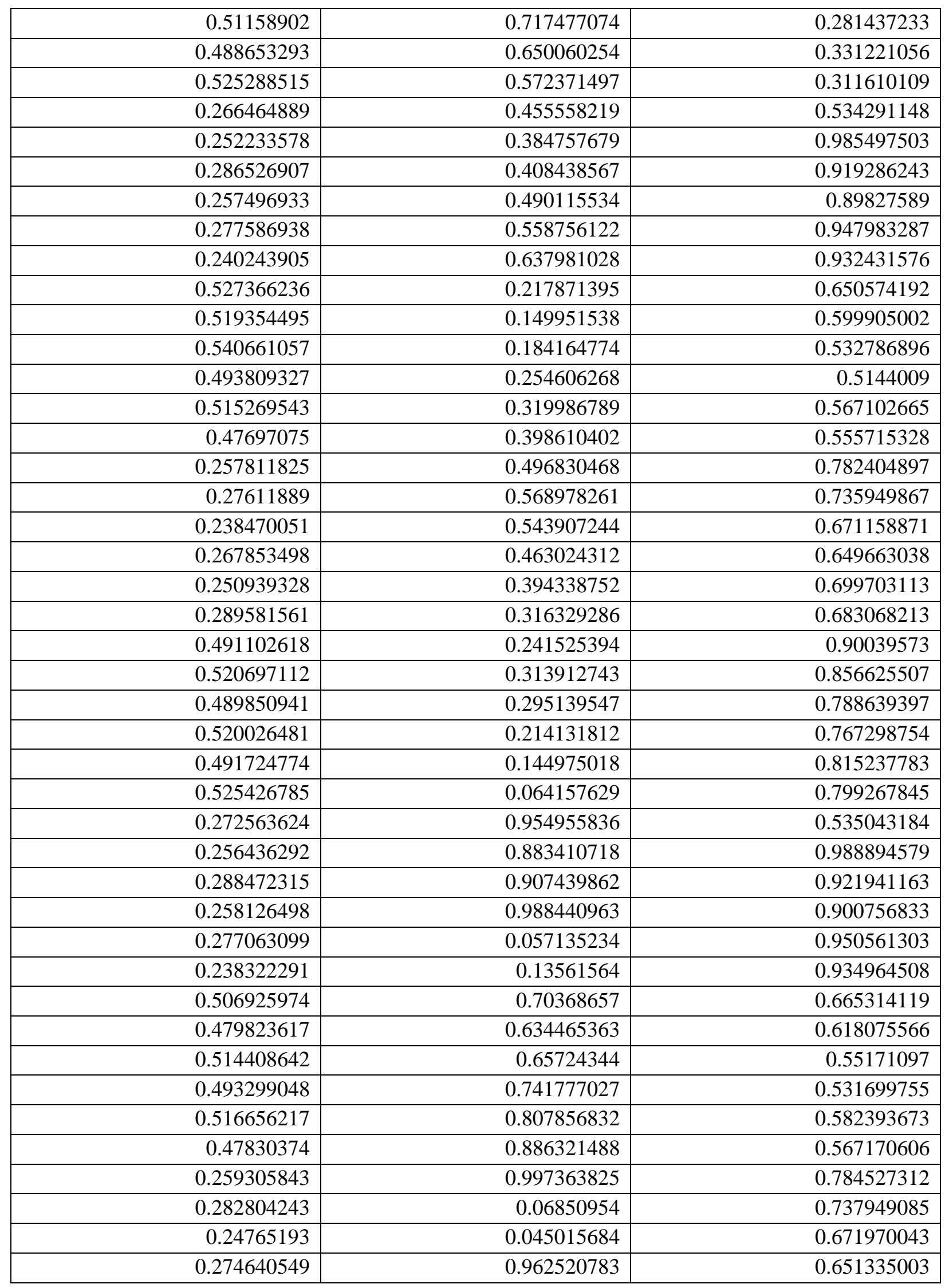




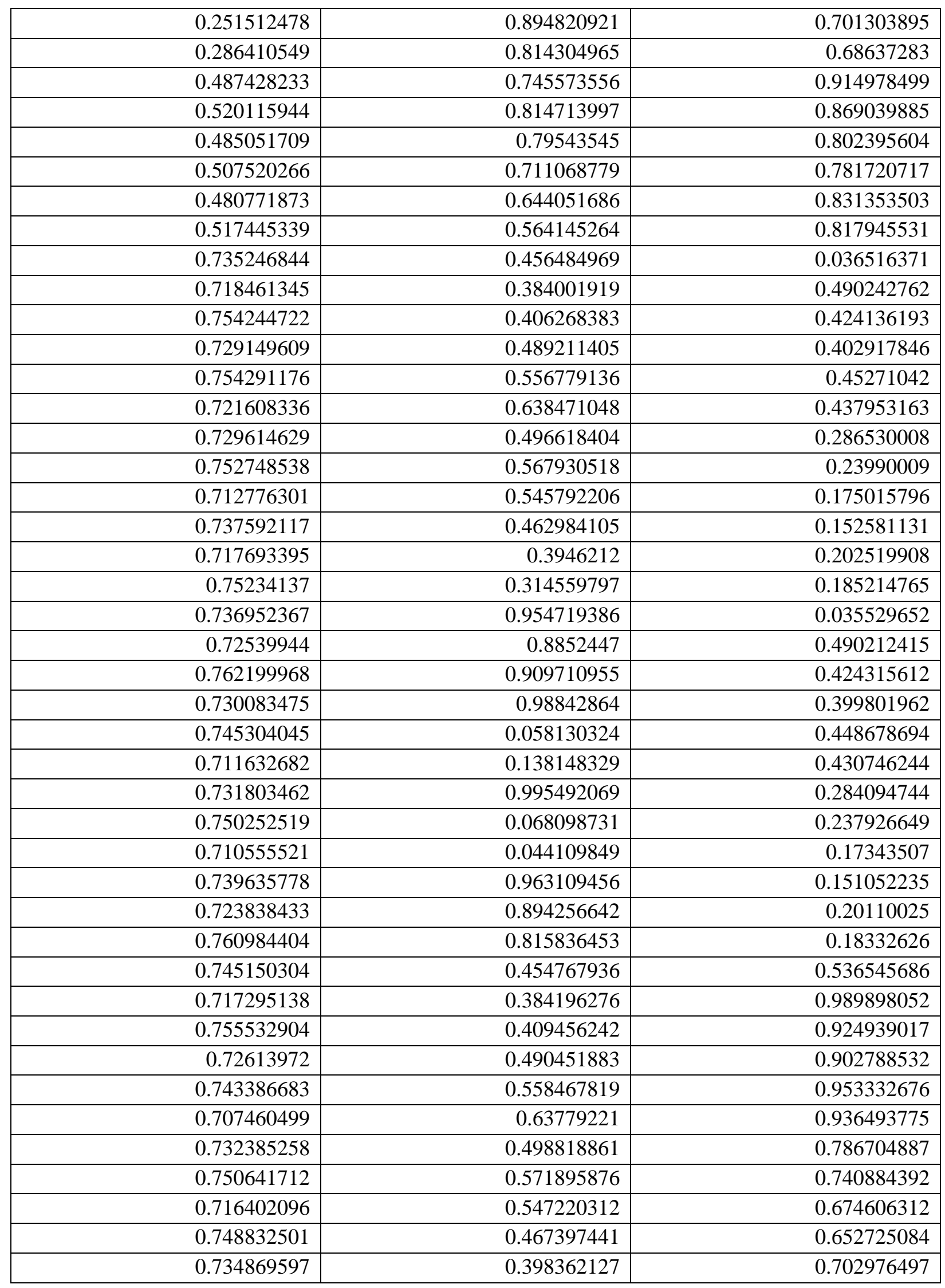




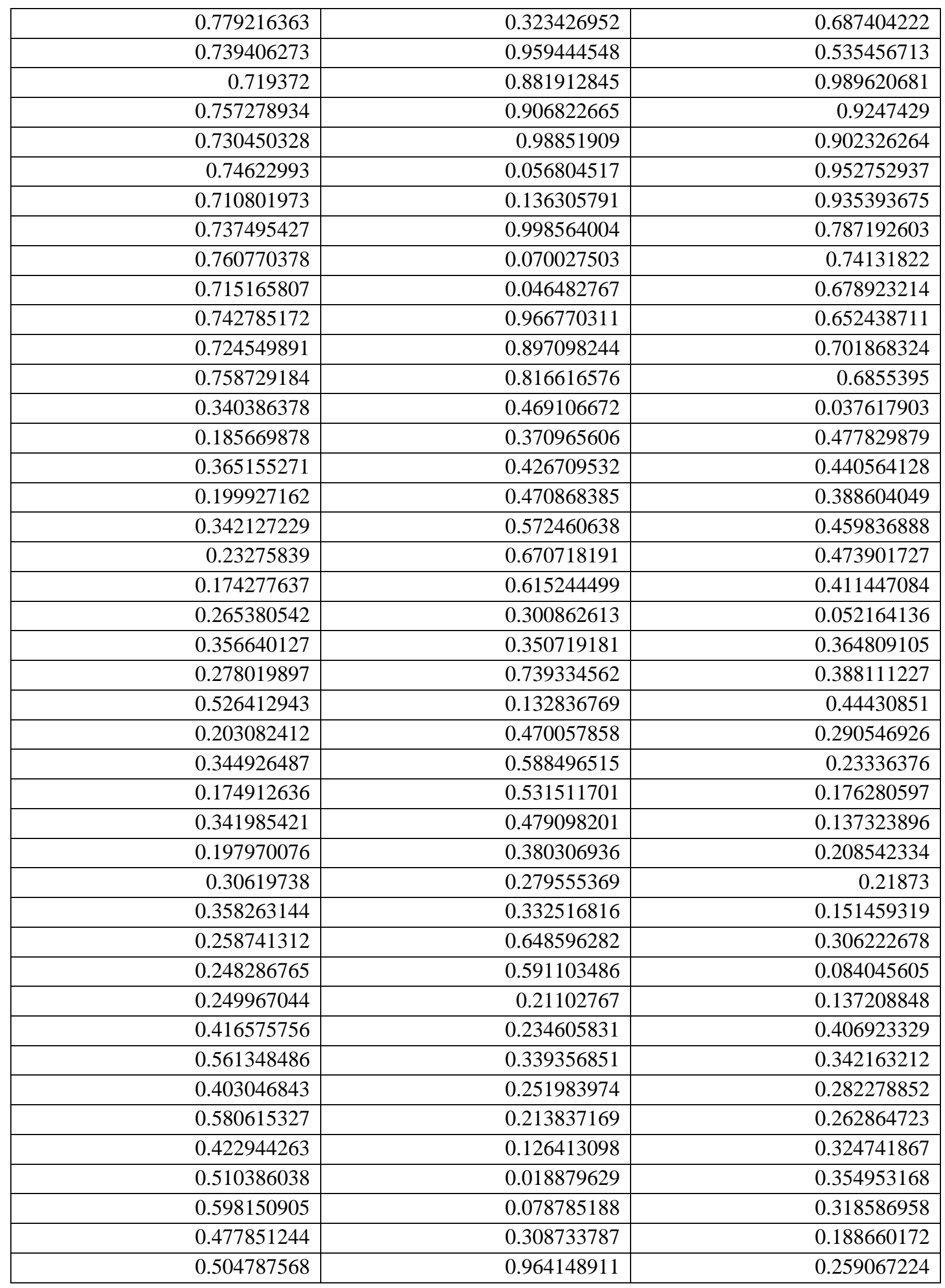




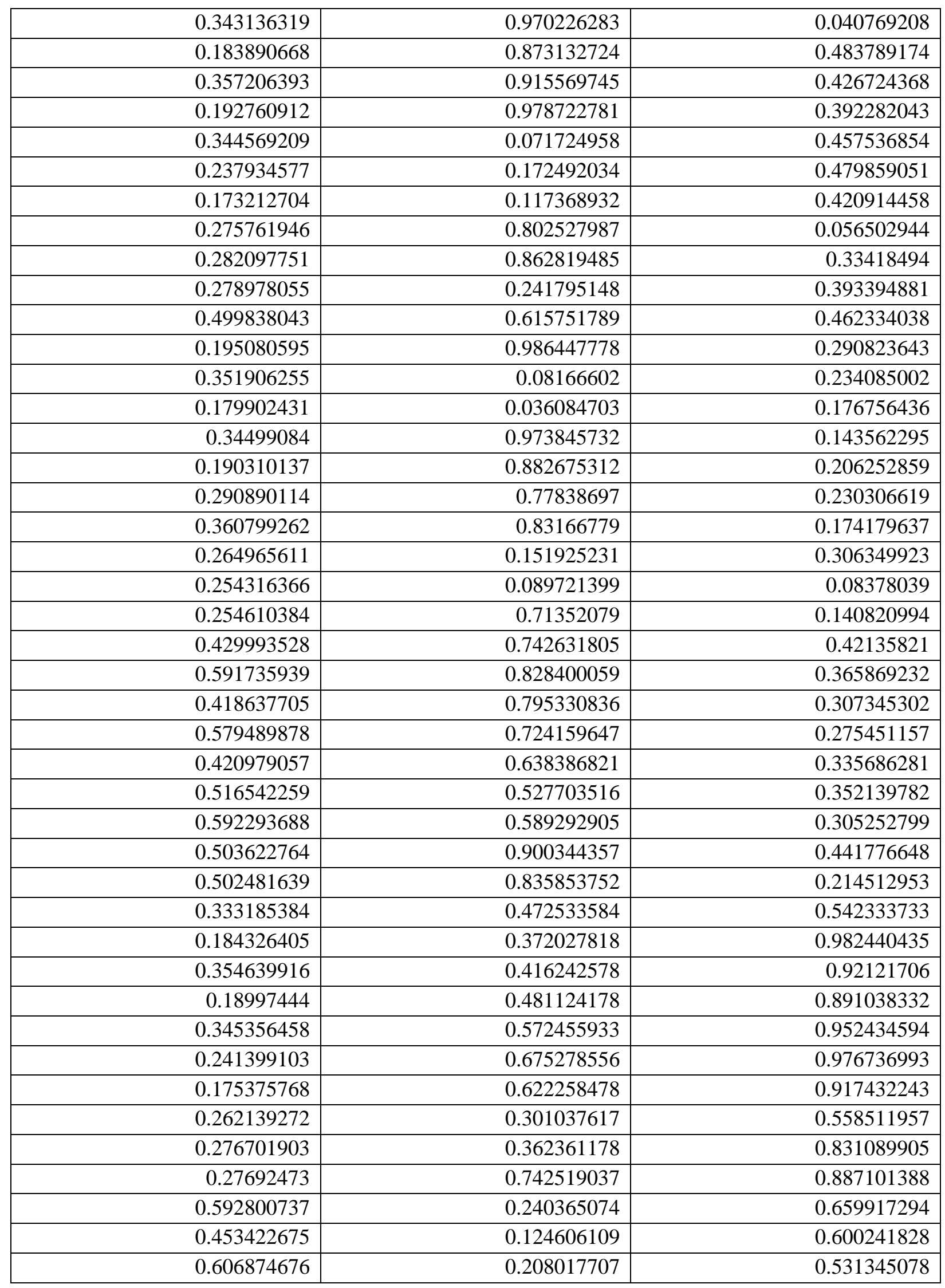




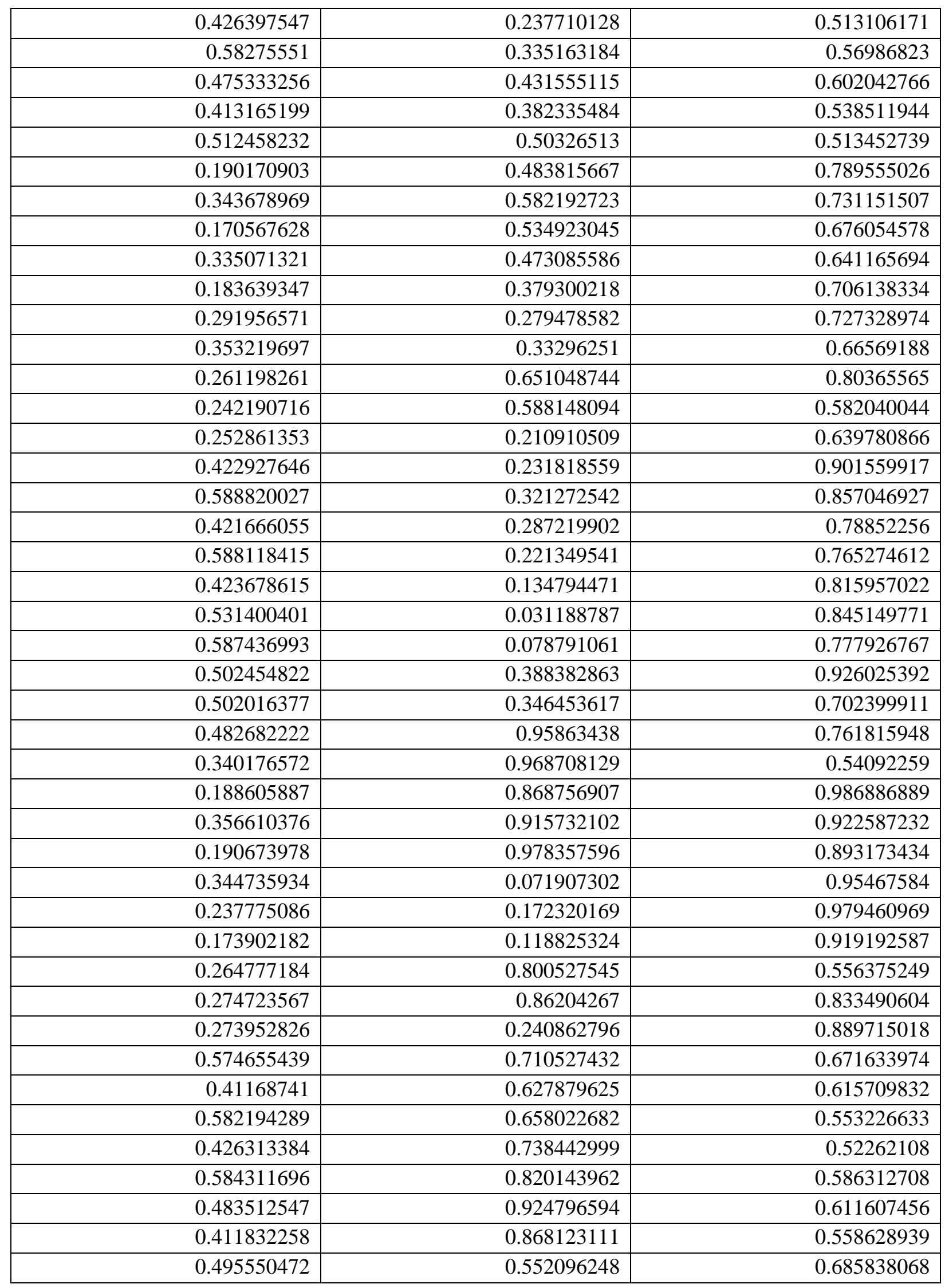




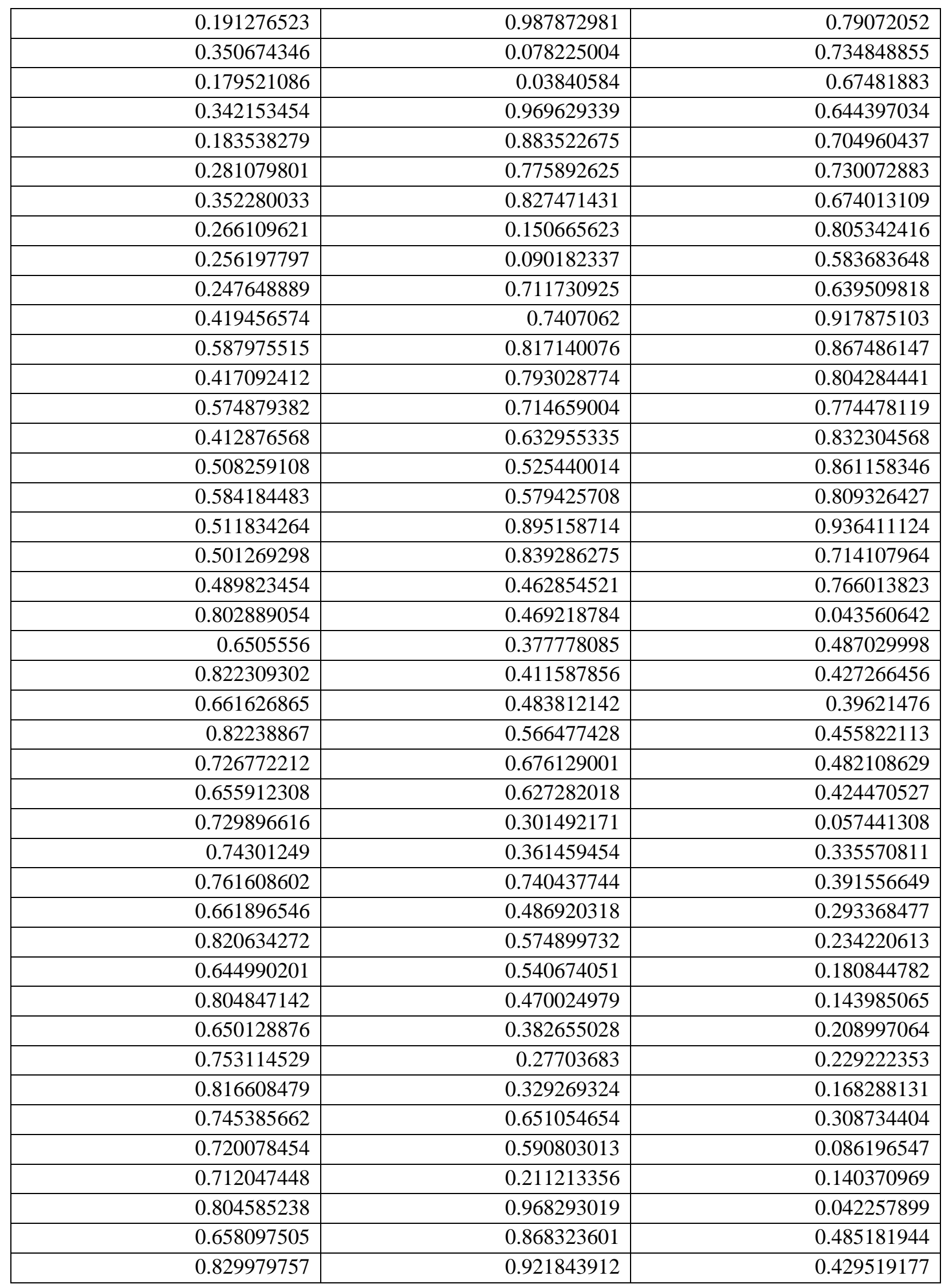




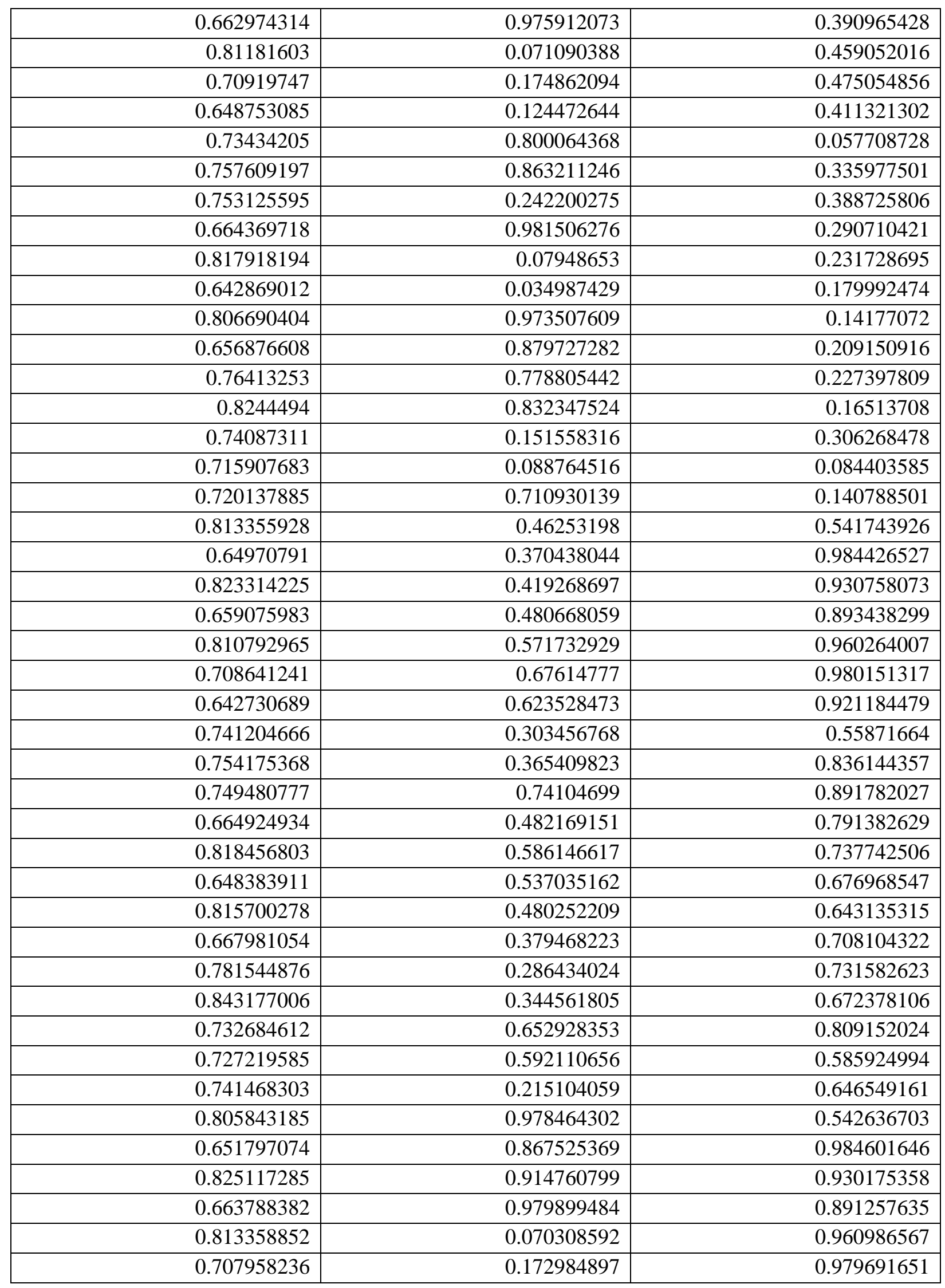




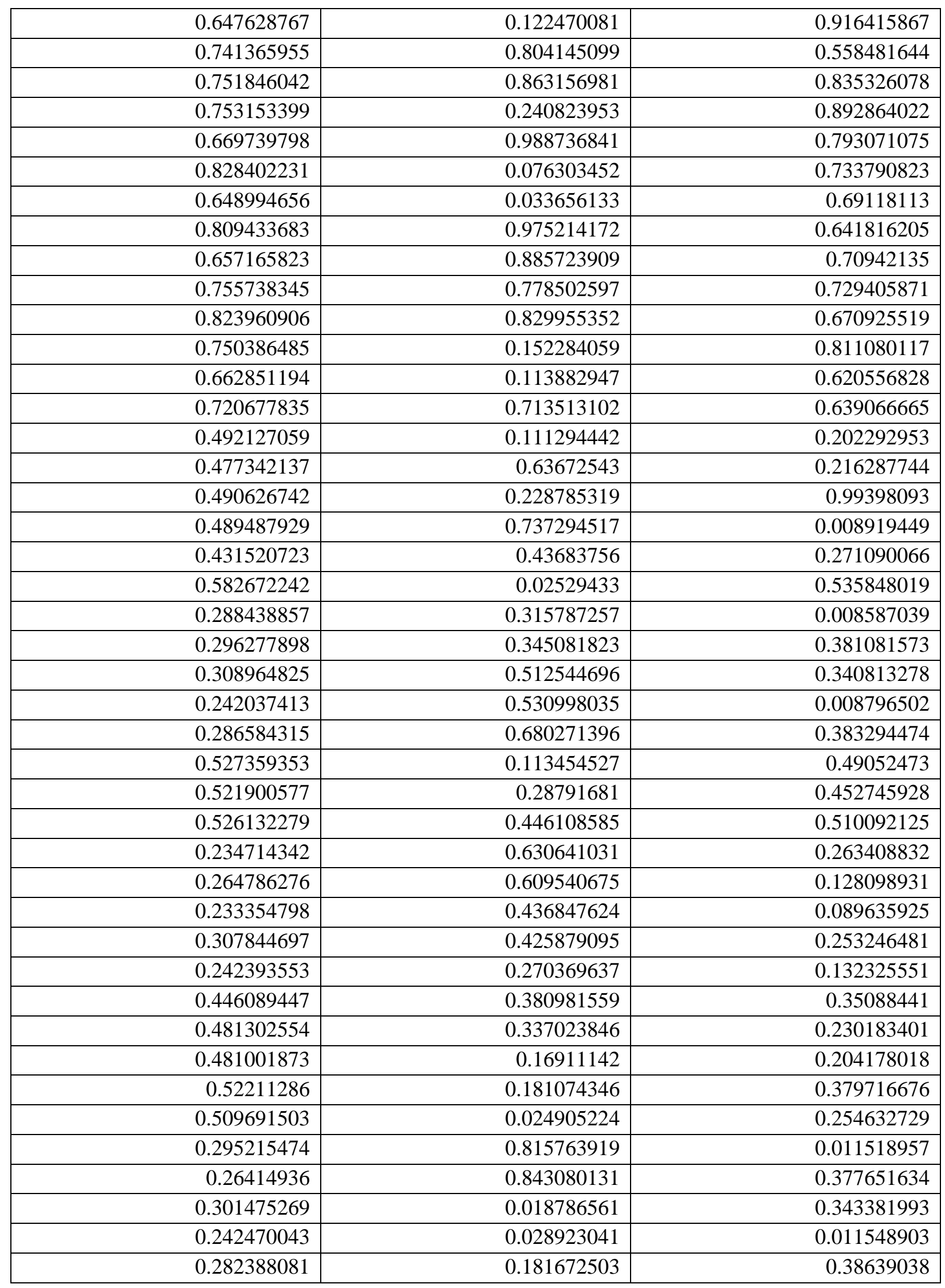




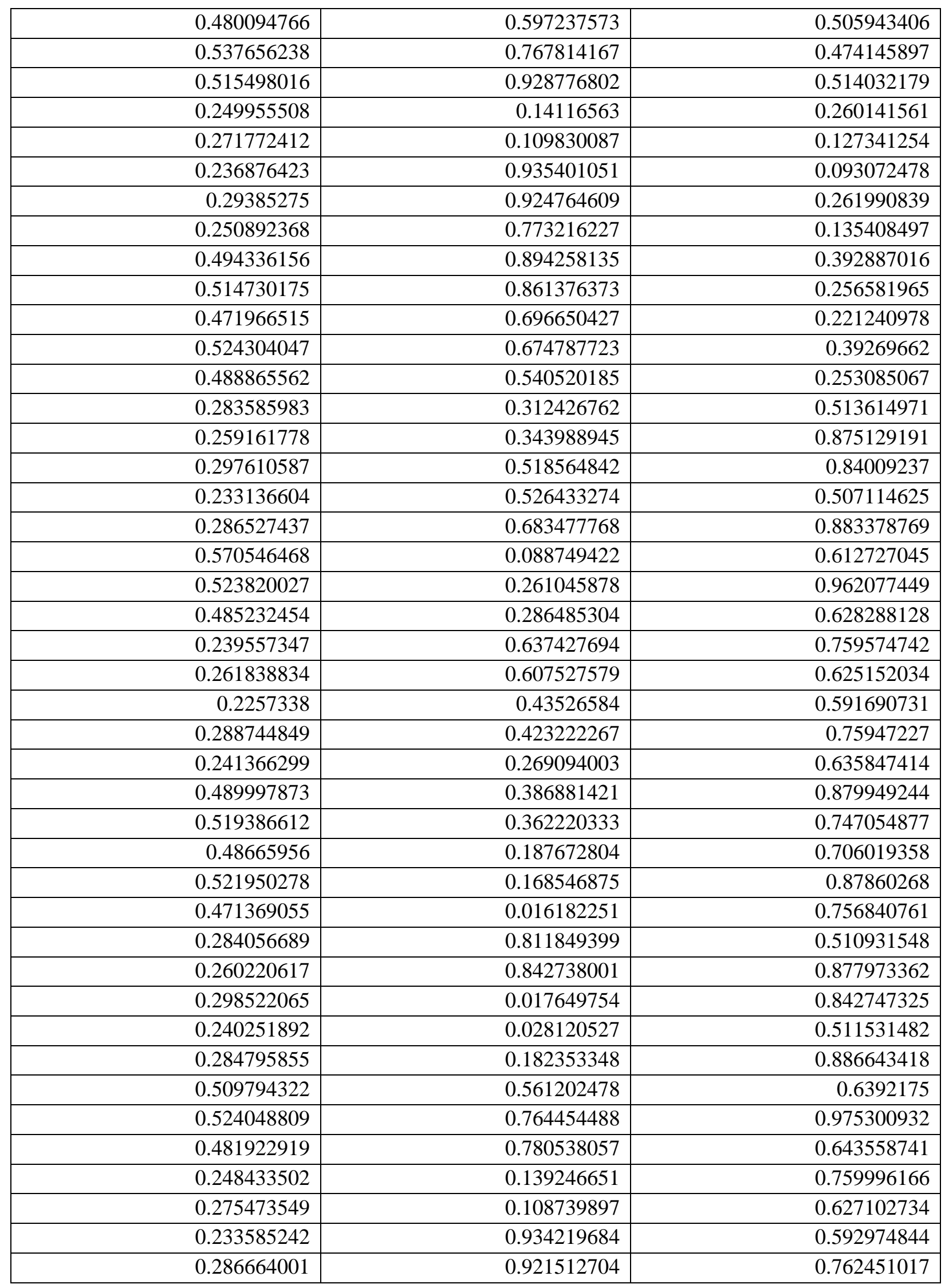




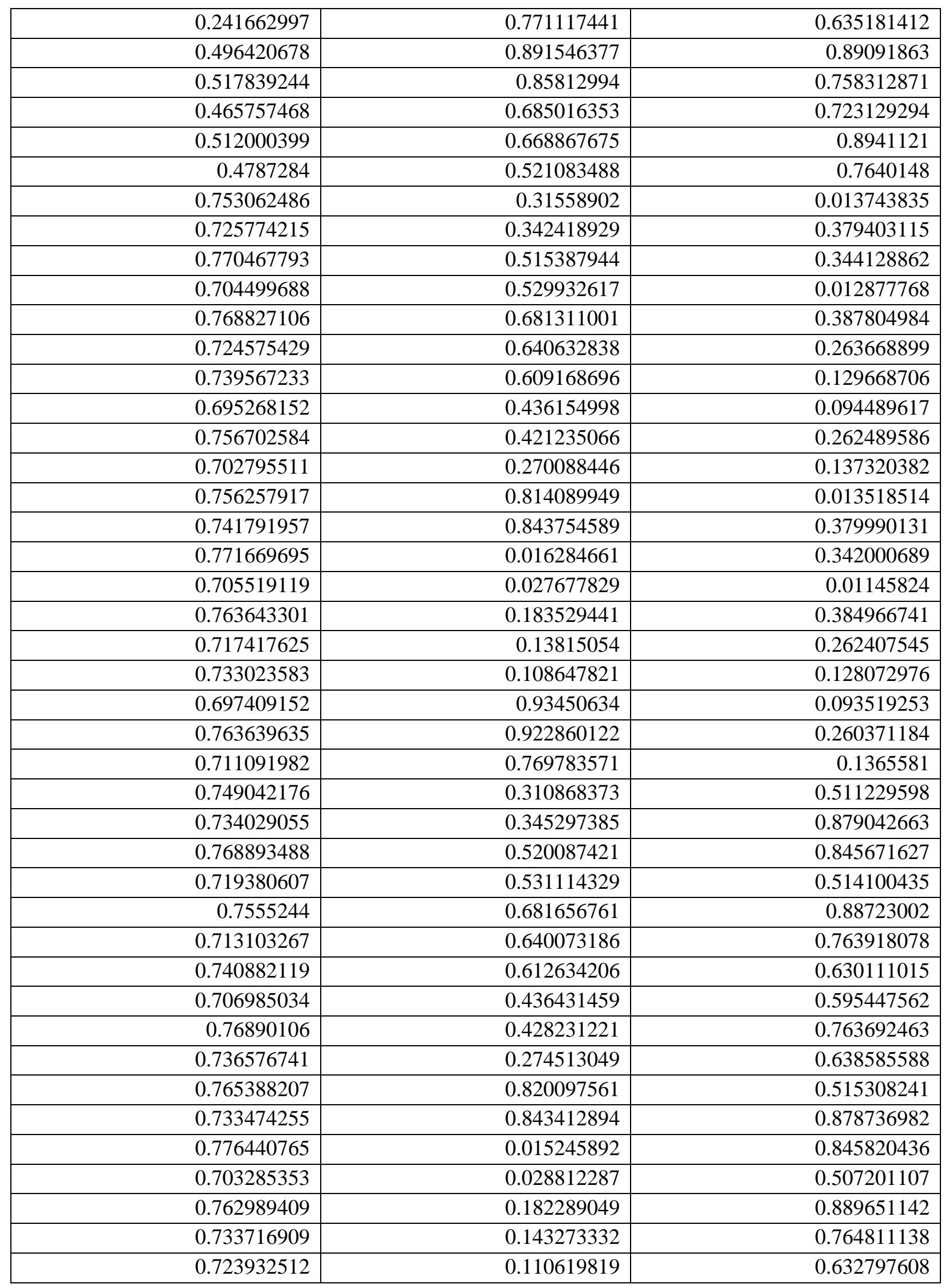




\begin{tabular}{|r|r|r|}
\hline 0.698397726 & 0.942235525 & 0.593539095 \\
\hline 0.76469172 & 0.92363962 & 0.761718541 \\
\hline 0.711415589 & 0.772702916 & 0.635826167 \\
\hline
\end{tabular}

Mg-Cell-Mg boat conformer co-ordinates:

$\mathrm{a}=16.352 \AA, \mathrm{b}=16.402 \AA, \mathrm{c}=20.760 \AA$

$\Upsilon=96.550$

$\begin{array}{llll}\mathrm{C} & \mathrm{H} & \mathrm{Mg} & \mathrm{O}\end{array}$

$\begin{array}{llll}132 & 220 & 2 & 112\end{array}$

\begin{tabular}{|c|c|c|}
\hline $\mathrm{x}$ & $\mathrm{y}$ & $\mathrm{z}$ \\
\hline 0.274339306 & 0.45624614 & 0.032862426 \\
\hline 0.242280251 & 0.382996685 & 0.489346388 \\
\hline 0.293028433 & 0.407700451 & 0.429541967 \\
\hline 0.263046641 & 0.485661515 & 0.401427418 \\
\hline 0.27577489 & 0.556380905 & 0.450432458 \\
\hline 0.239386679 & 0.634694258 & 0.430997102 \\
\hline 0.266742481 & 0.492102664 & 0.28472005 \\
\hline 0.278744629 & 0.56677319 & 0.239708446 \\
\hline 0.243970016 & 0.542626244 & 0.173189237 \\
\hline 0.276369954 & 0.464076776 & 0.148804069 \\
\hline 0.263003529 & 0.393867464 & 0.197191445 \\
\hline 0.298123335 & 0.315229273 & 0.176312977 \\
\hline 0.48110467 & 0.258956765 & 0.402605951 \\
\hline 0.491058305 & 0.319656067 & 0.346392182 \\
\hline 0.466846947 & 0.274720682 & 0.283691907 \\
\hline 0.510890676 & 0.200949519 & 0.270186223 \\
\hline 0.489869399 & 0.143710689 & 0.327857624 \\
\hline 0.530316357 & 0.06480883 & 0.324104066 \\
\hline 0.275780968 & 0.955912776 & 0.034549675 \\
\hline 0.250331121 & 0.881361344 & 0.487348153 \\
\hline 0.28873653 & 0.90617351 & 0.422381686 \\
\hline 0.259697375 & 0.987572875 & 0.400721185 \\
\hline 0.275971393 & 0.055552902 & 0.451454555 \\
\hline 0.236580333 & 0.133487133 & 0.435619517 \\
\hline 0.263281468 & 0.997655087 & 0.284745082 \\
\hline 0.283825403 & 0.069559712 & 0.238250683 \\
\hline 0.247338405 & 0.044634559 & 0.172733963 \\
\hline 0.277777683 & 0.963866233 & 0.151002961 \\
\hline 0.258728732 & 0.895638846 & 0.201258077 \\
\hline 0.295937661 & 0.816280122 & 0.185970052 \\
\hline 0.499668777 & 0.743993688 & 0.41250621 \\
\hline
\end{tabular}

Facas et al. 


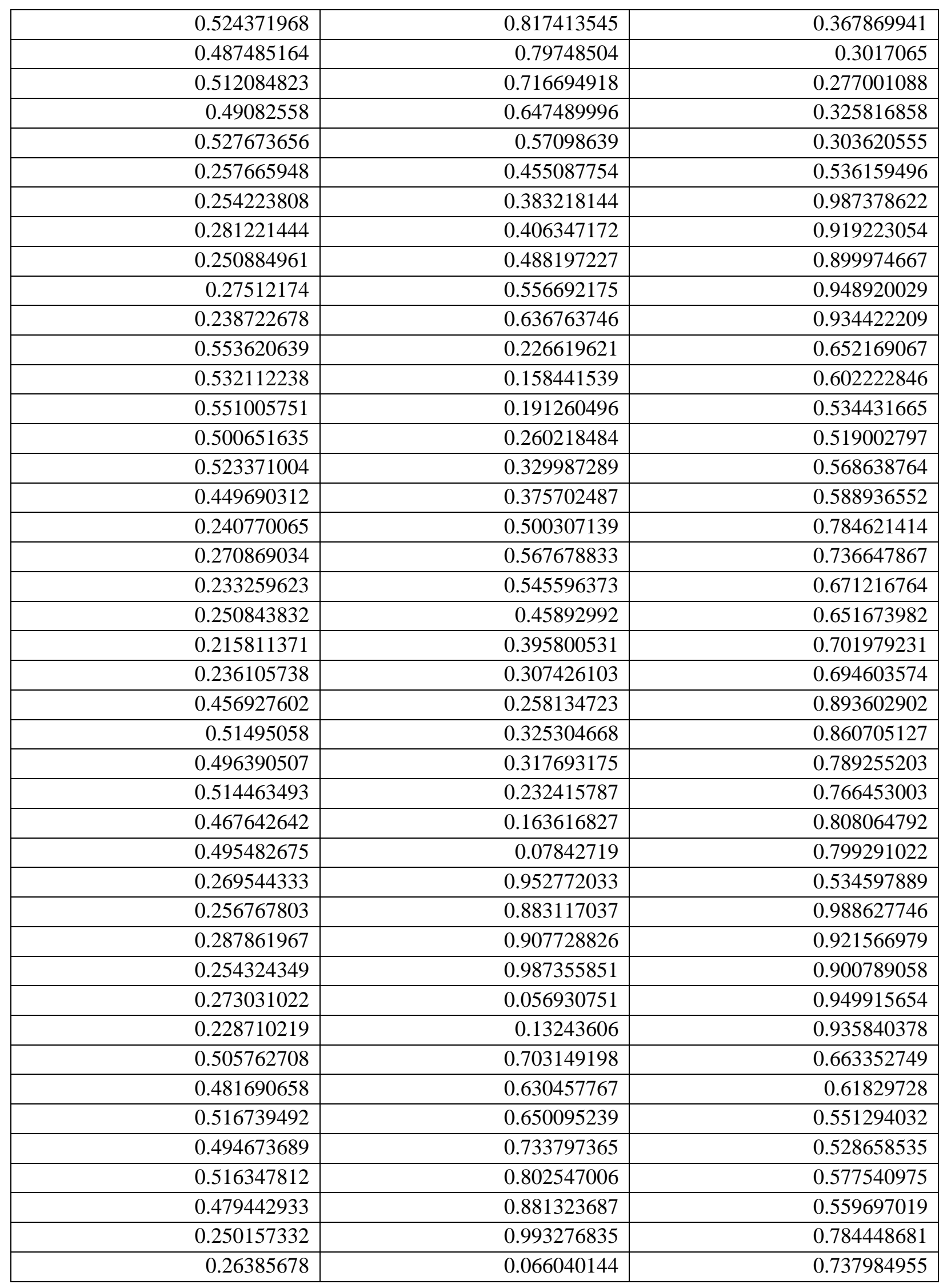




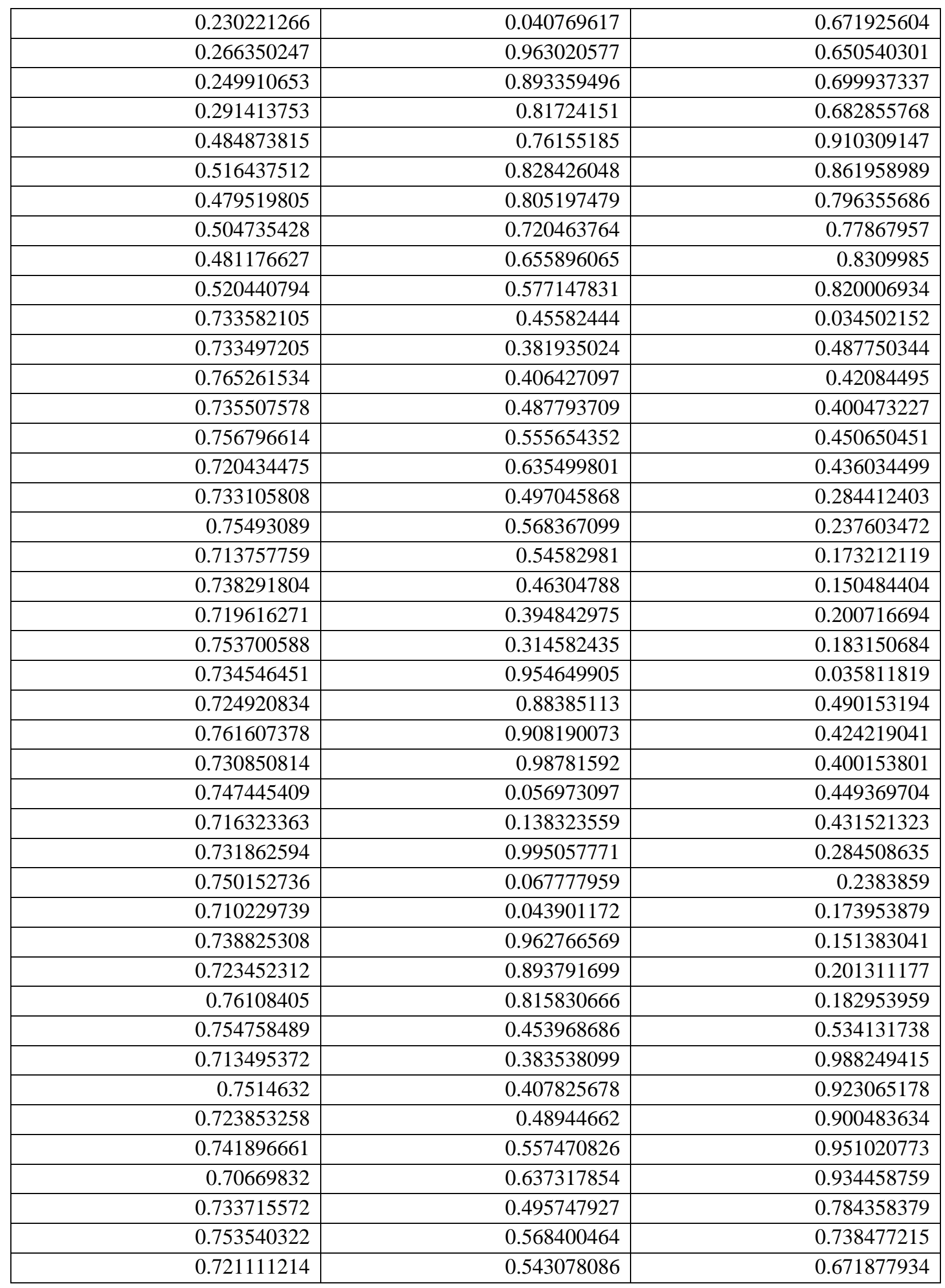




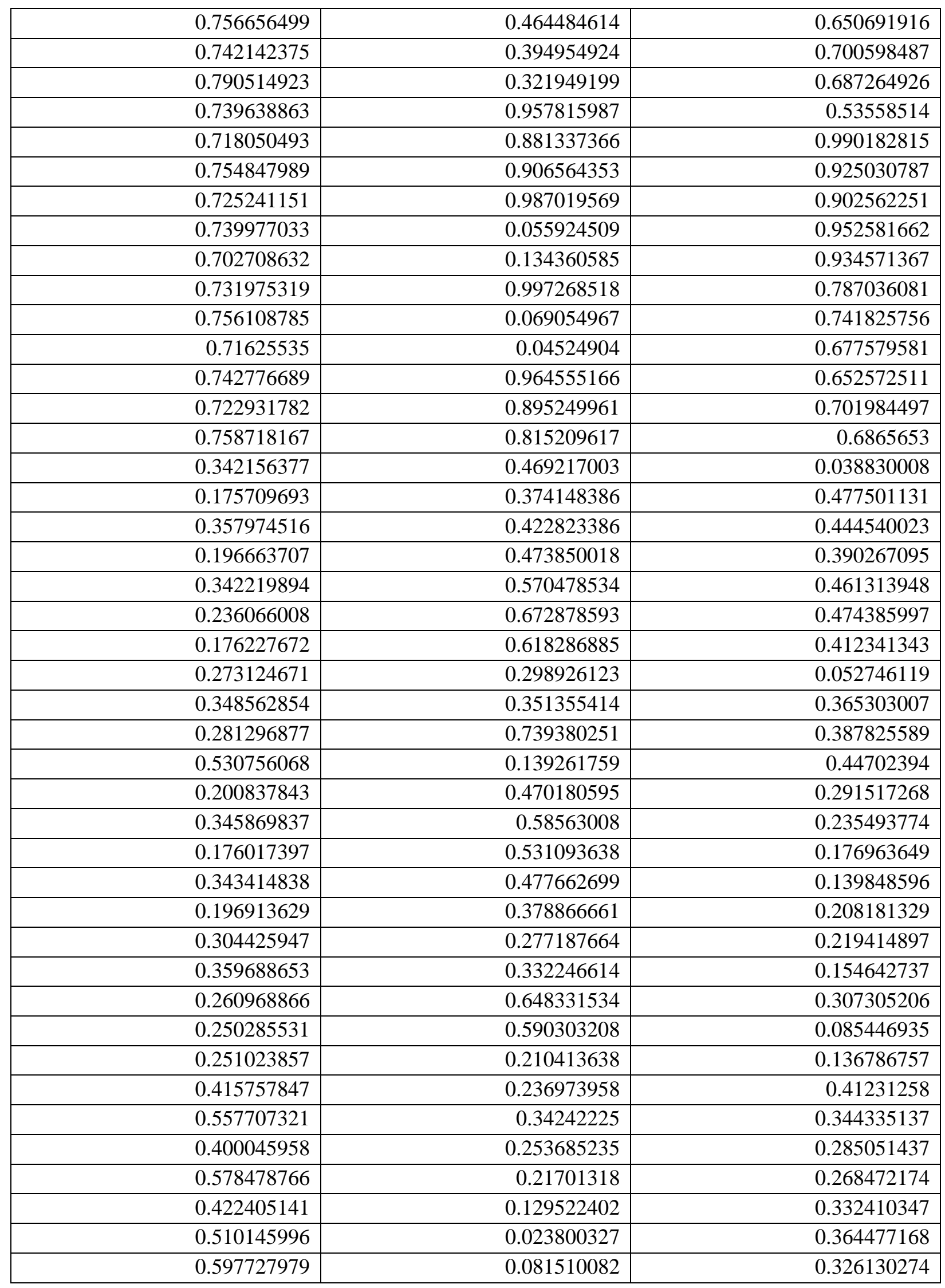




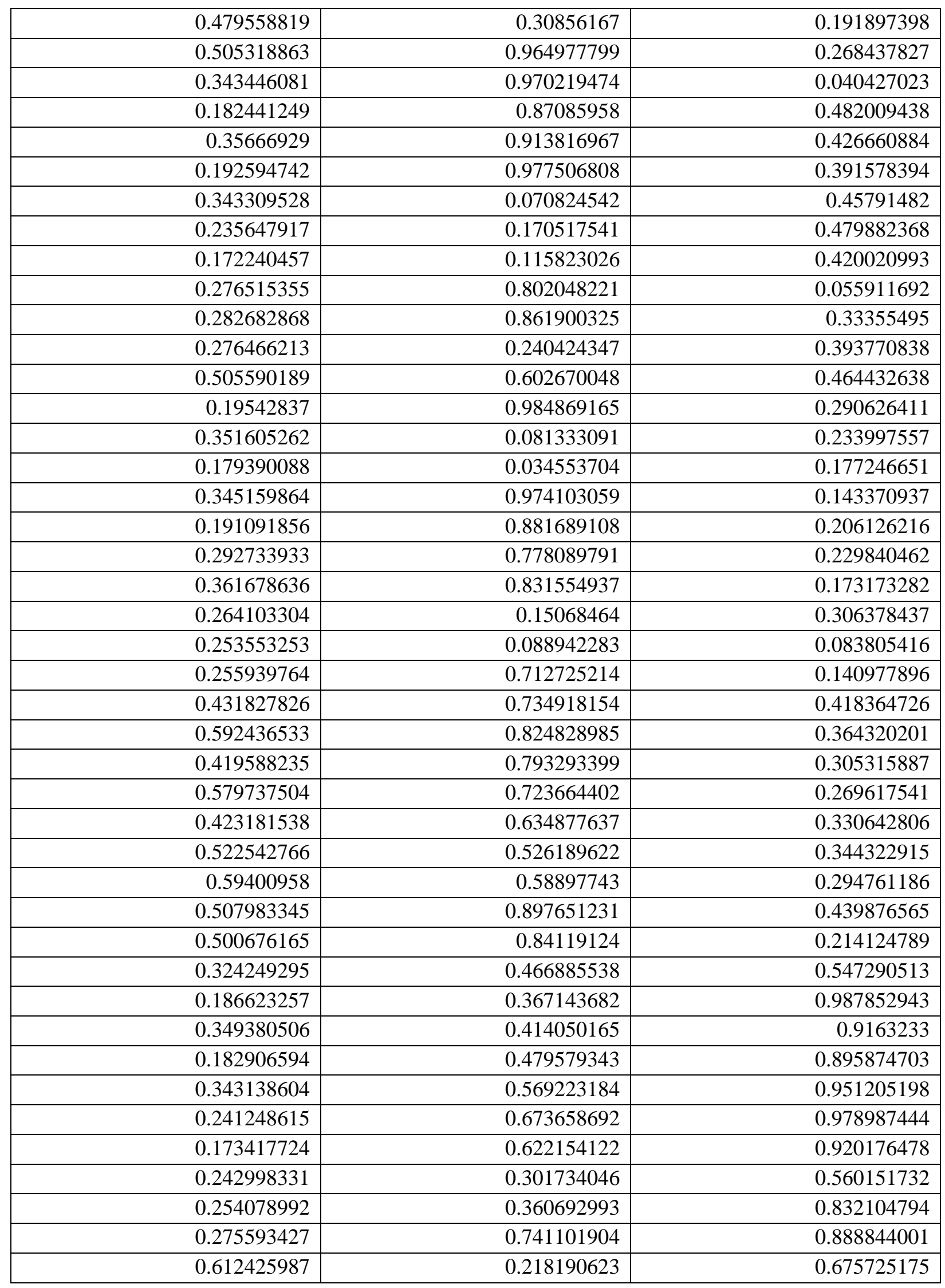




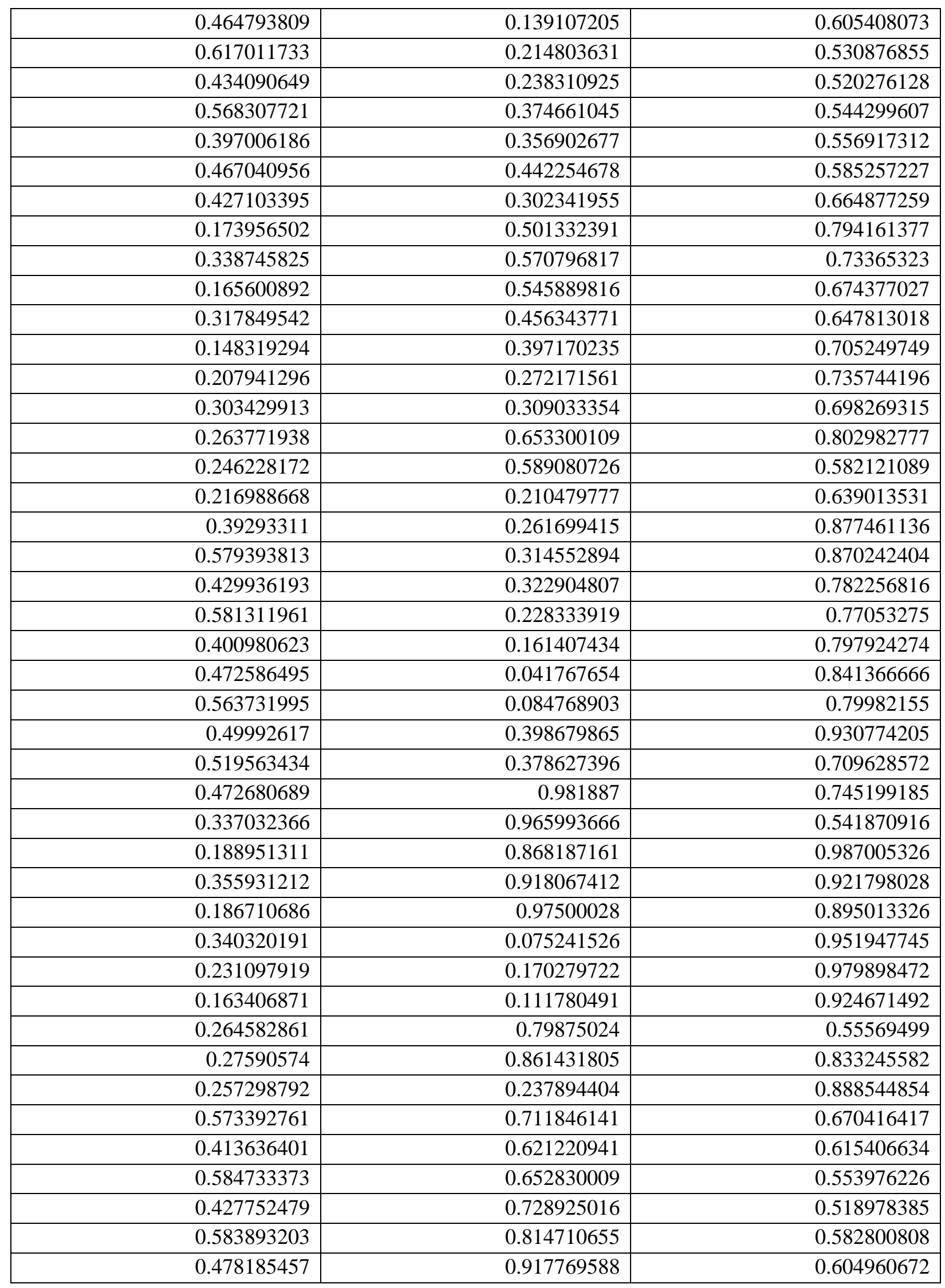




\begin{tabular}{|c|c|c|}
\hline 0.41459581 & 0.863432321 & 0.545499814 \\
\hline 0.497508246 & 0.552872952 & 0.689069344 \\
\hline 0.183114105 & 0.97707481 & 0.792893631 \\
\hline 0.331041683 & 0.083151156 & 0.733899656 \\
\hline 0.162390002 & 0.02669177 & 0.674995221 \\
\hline 0.333635394 & 0.977148174 & 0.644042241 \\
\hline 0.182598061 & 0.876227969 & 0.704383867 \\
\hline 0.293984417 & 0.779501943 & 0.726553859 \\
\hline 0.355088842 & 0.836176791 & 0.666742114 \\
\hline 0.242112561 & 0.145764208 & 0.805730479 \\
\hline 0.239561813 & 0.087120359 & 0.583893379 \\
\hline 0.254171404 & 0.711171925 & 0.639765059 \\
\hline 0.416780756 & 0.75479442 & 0.9123838 \\
\hline 0.584302517 & 0.830516374 & 0.859599386 \\
\hline 0.411483677 & 0.800212564 & 0.799937361 \\
\hline 0.572200423 & 0.726221907 & 0.771431382 \\
\hline 0.413399559 & 0.642275612 & 0.832546897 \\
\hline 0.515488733 & 0.542272912 & 0.865365239 \\
\hline 0.586249885 & 0.593285525 & 0.808208972 \\
\hline 0.509231724 & 0.911540317 & 0.927272819 \\
\hline 0.493815944 & 0.843206674 & 0.70662374 \\
\hline 0.499557301 & 0.473768212 & 0.771878395 \\
\hline 0.801440293 & 0.467670142 & 0.040653787 \\
\hline 0.66557803 & 0.369447924 & 0.485199342 \\
\hline 0.833426099 & 0.414787276 & 0.422078827 \\
\hline 0.667894386 & 0.478912993 & 0.393918505 \\
\hline 0.824617833 & 0.568586056 & 0.454663469 \\
\hline 0.723256501 & 0.672713646 & 0.480414236 \\
\hline 0.655171323 & 0.621811612 & 0.421889747 \\
\hline 0.72623906 & 0.300806066 & 0.056024844 \\
\hline 0.751575018 & 0.361181806 & 0.332417556 \\
\hline 0.758517419 & 0.739276973 & 0.390998523 \\
\hline 0.665523279 & 0.48695421 & 0.292055846 \\
\hline 0.822698033 & 0.575613263 & 0.231109385 \\
\hline 0.646056617 & 0.54048873 & 0.1797703 \\
\hline 0.805361175 & 0.470234829 & 0.140998316 \\
\hline 0.65221678 & 0.382987023 & 0.208233932 \\
\hline 0.755574838 & 0.277411279 & 0.227286506 \\
\hline 0.817518205 & 0.329060839 & 0.165140388 \\
\hline 0.746363648 & 0.65088172 & 0.307023006 \\
\hline 0.719390671 & 0.590674325 & 0.08430229 \\
\hline 0.711628333 & 0.210873347 & 0.139644485 \\
\hline 0.802093711 & 0.969769179 & 0.042109182 \\
\hline
\end{tabular}




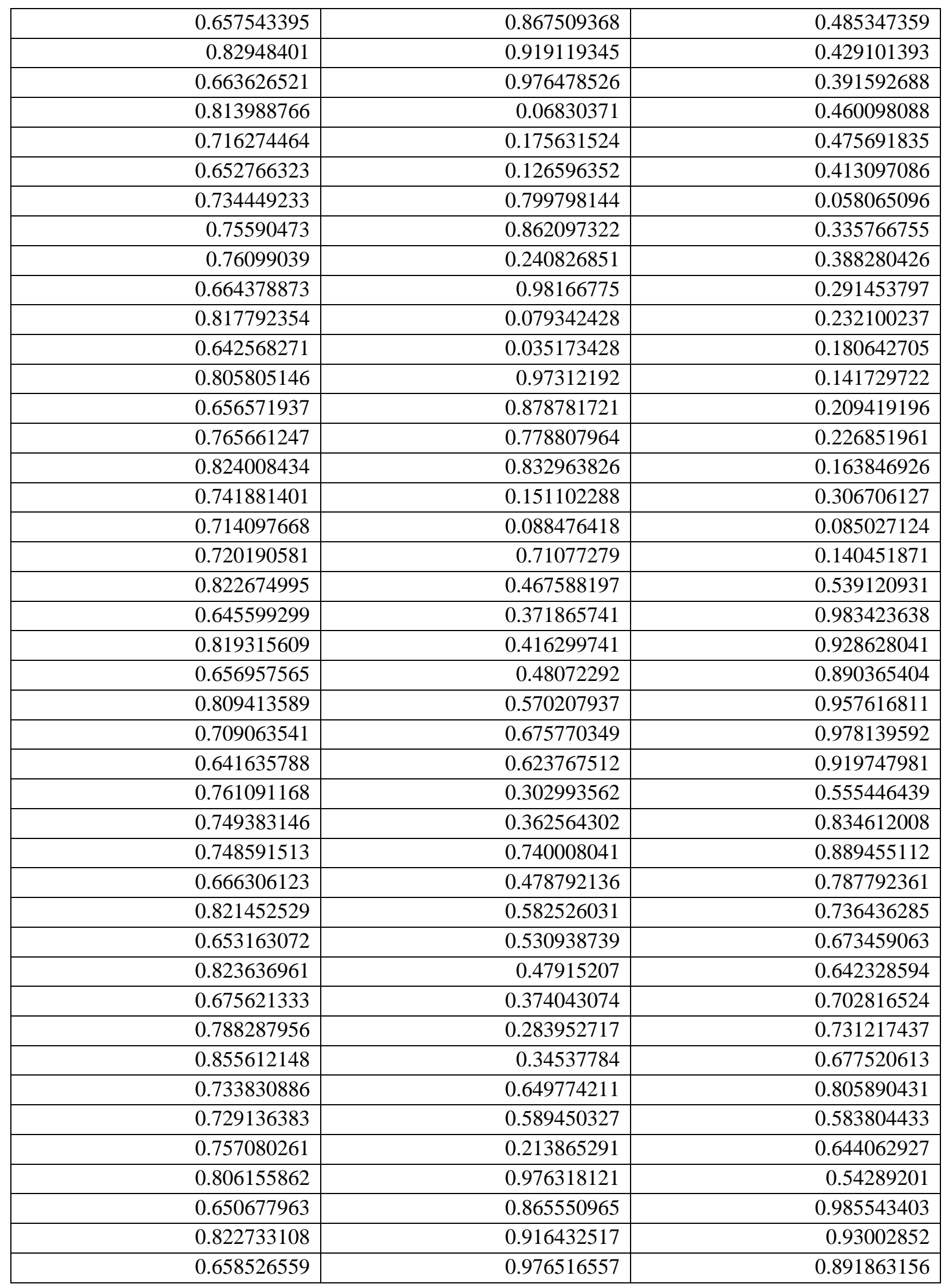




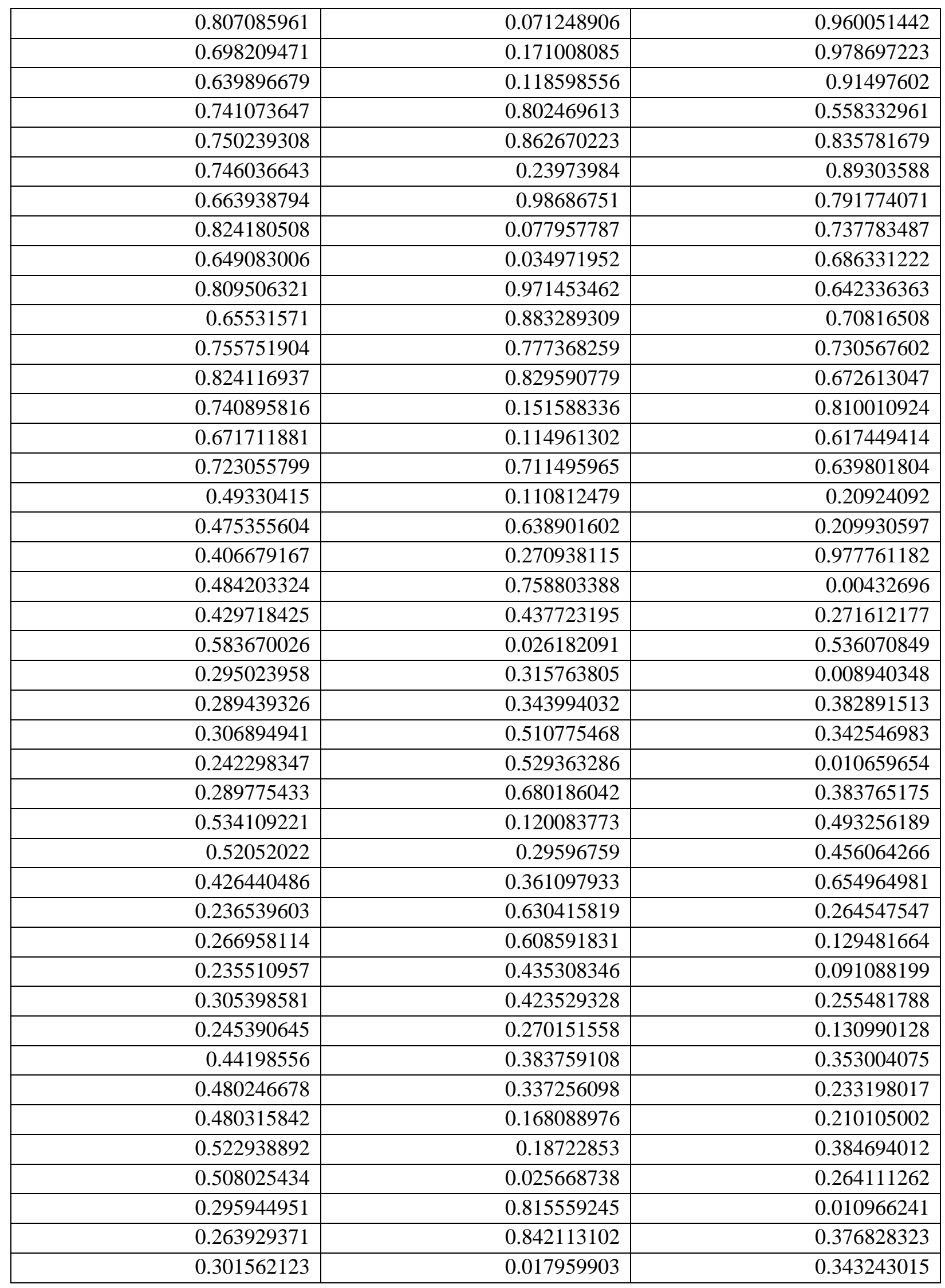




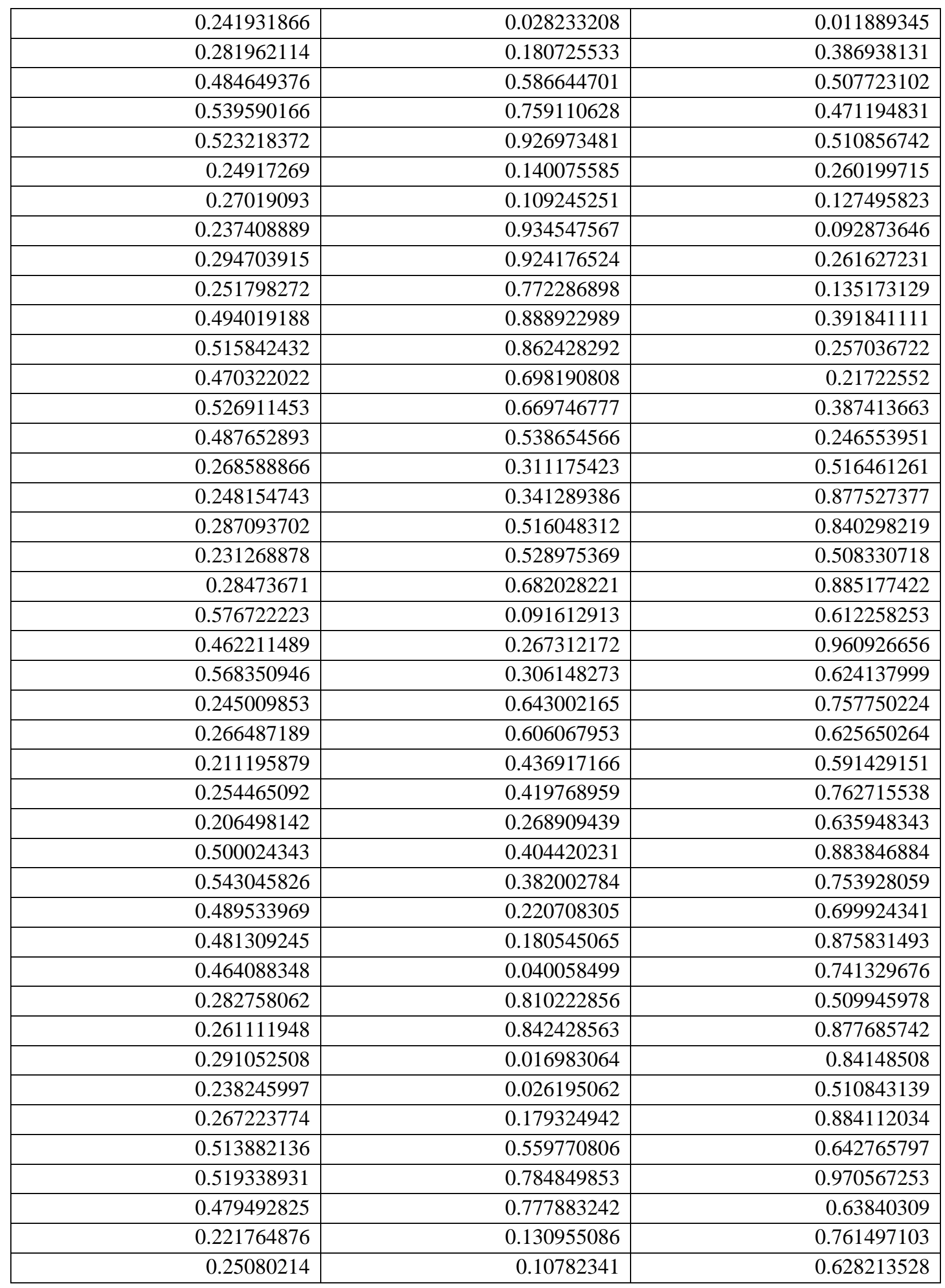




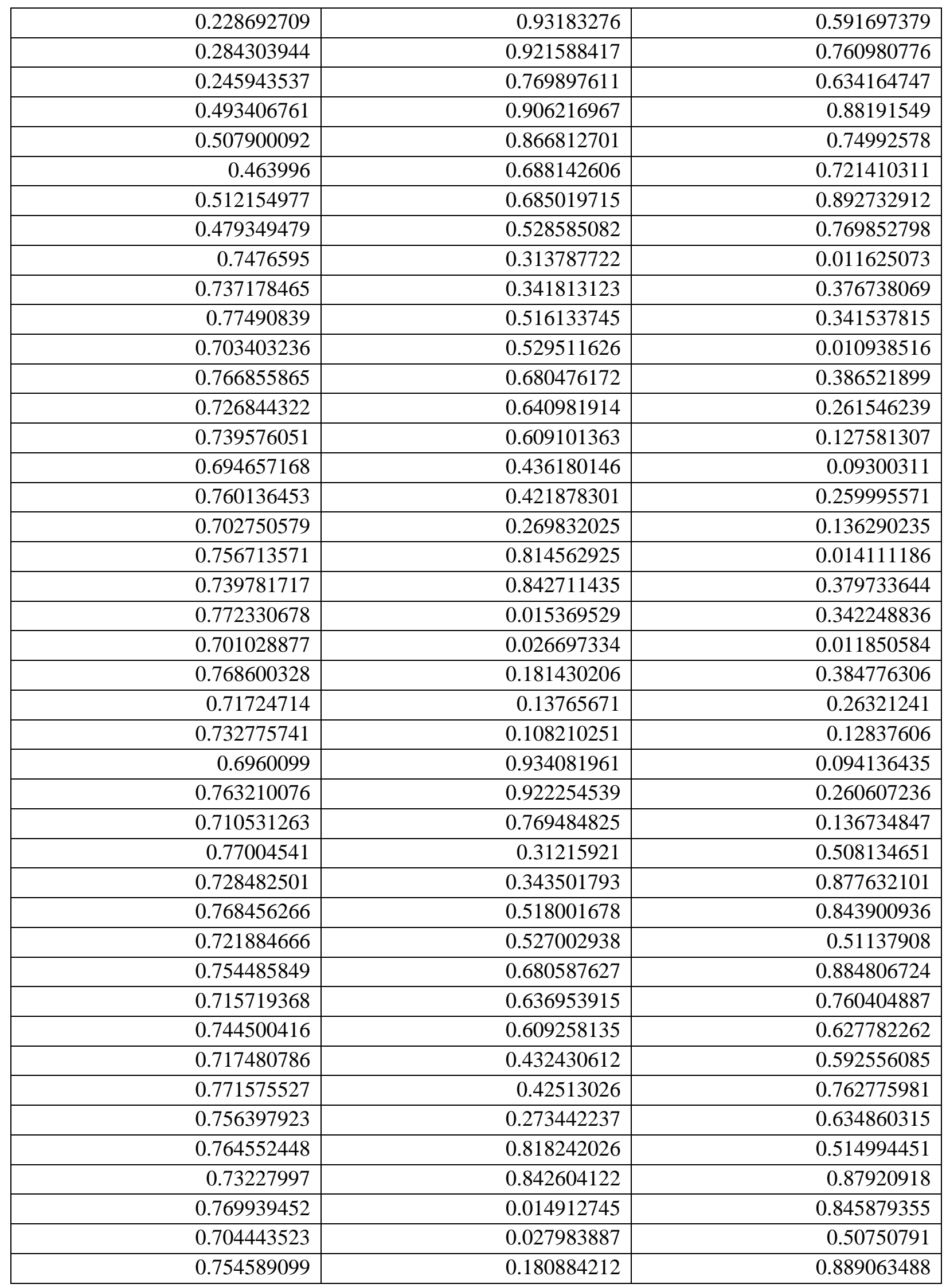




\begin{tabular}{|r|r|r|}
\hline 0.724292155 & 0.140827133 & 0.763978733 \\
\hline 0.731190416 & 0.108988099 & 0.631273619 \\
\hline 0.698525446 & 0.940399173 & 0.593542517 \\
\hline 0.760919191 & 0.922687072 & 0.762404975 \\
\hline 0.712982562 & 0.770372474 & 0.636516017 \\
\hline
\end{tabular}

Mg-Cell-Mg Transition State co-ordinates:

$\mathrm{a}=16.352 \AA, \mathrm{b}=16.402 \AA, \mathrm{c}=20.760 \AA$

$\Upsilon=96.550$

C $\quad \mathrm{H} \quad \mathrm{Mg} \quad \mathrm{O}$

$\begin{array}{llll}132 & 220 & 2 & 112\end{array}$

\begin{tabular}{|c|c|c|}
\hline $\mathrm{x}$ & $y$ & $\mathrm{z}$ \\
\hline 0.277471531 & 0.457016956 & 0.030721349 \\
\hline 0.249280614 & 0.382993542 & 0.487720827 \\
\hline 0.298971702 & 0.408310563 & 0.427540526 \\
\hline 0.267556762 & 0.485506457 & 0.399275492 \\
\hline 0.278265861 & 0.556558868 & 0.448103546 \\
\hline 0.240236931 & 0.633788531 & 0.428117677 \\
\hline 0.272471746 & 0.492570005 & 0.282627733 \\
\hline 0.284118615 & 0.567280036 & 0.23764544 \\
\hline 0.249633667 & 0.542948516 & 0.171096752 \\
\hline 0.281672413 & 0.464267174 & 0.146624628 \\
\hline 0.268813245 & 0.394323904 & 0.195221111 \\
\hline 0.303751098 & 0.315511455 & 0.174731172 \\
\hline 0.48208629 & 0.259443003 & 0.398829704 \\
\hline 0.494717075 & 0.318904396 & 0.342337791 \\
\hline 0.471966605 & 0.273886927 & 0.279634638 \\
\hline 0.516185432 & 0.199760964 & 0.267985913 \\
\hline 0.492679254 & 0.143134257 & 0.325735548 \\
\hline 0.533229495 & 0.064205874 & 0.323905454 \\
\hline 0.278112248 & 0.956417252 & 0.032923169 \\
\hline 0.25169103 & 0.881824485 & 0.48595952 \\
\hline 0.290440435 & 0.906503494 & 0.42108454 \\
\hline 0.262246758 & 0.988139173 & 0.399032805 \\
\hline 0.27807266 & 0.056033046 & 0.44989255 \\
\hline 0.239397896 & 0.13426903 & 0.433617186 \\
\hline 0.267944611 & 0.998080994 & 0.283002208 \\
\hline 0.289259233 & 0.069825205 & 0.236514479 \\
\hline 0.252628544 & 0.044889303 & 0.171048409 \\
\hline 0.282525404 & 0.963867714 & 0.14934261 \\
\hline 0.26391395 & 0.895761413 & 0.199761181 \\
\hline
\end{tabular}

Facas et al. 


\begin{tabular}{|c|c|c|}
\hline 0.301785114 & 0.816697404 & 0.184655811 \\
\hline 0.497714811 & 0.7462065 & 0.418344725 \\
\hline 0.524824434 & 0.818546177 & 0.37329771 \\
\hline 0.488787442 & 0.798185762 & 0.306968347 \\
\hline 0.513636948 & 0.717049884 & 0.283346951 \\
\hline 0.489912394 & 0.648184958 & 0.331838057 \\
\hline 0.528147163 & 0.571713104 & 0.311429811 \\
\hline 0.262462381 & 0.45576402 & 0.534067044 \\
\hline 0.257763855 & 0.383680387 & 0.985582021 \\
\hline 0.283564844 & 0.407075777 & 0.917240577 \\
\hline 0.251638983 & 0.488333002 & 0.898201492 \\
\hline 0.275296083 & 0.557220745 & 0.946981799 \\
\hline 0.237086 & 0.636480829 & 0.932911942 \\
\hline 0.569620735 & 0.241584123 & 0.638729057 \\
\hline 0.533070815 & 0.168346978 & 0.600651551 \\
\hline 0.543604622 & 0.193400561 & 0.529998261 \\
\hline 0.496702056 & 0.26524796 & 0.514875656 \\
\hline 0.521736587 & 0.335129062 & 0.564054671 \\
\hline 0.450861464 & 0.365235753 & 0.605261579 \\
\hline 0.242207957 & 0.498881047 & 0.78239762 \\
\hline 0.268496954 & 0.568332115 & 0.734994593 \\
\hline 0.232192283 & 0.545558974 & 0.669251722 \\
\hline 0.254240107 & 0.460730117 & 0.649481386 \\
\hline 0.223191621 & 0.39523079 & 0.699207874 \\
\hline 0.250021364 & 0.310131087 & 0.688560829 \\
\hline 0.456719973 & 0.258901677 & 0.913930865 \\
\hline 0.508196468 & 0.323644267 & 0.873219245 \\
\hline 0.482428188 & 0.307054437 & 0.80376876 \\
\hline 0.503472786 & 0.220436998 & 0.786791437 \\
\hline 0.465981967 & 0.155187735 & 0.835542843 \\
\hline 0.501740482 & 0.073338508 & 0.830695346 \\
\hline 0.270832672 & 0.953452148 & 0.533015798 \\
\hline 0.259130812 & 0.883488943 & 0.987195503 \\
\hline 0.288363813 & 0.90837382 & 0.919783272 \\
\hline 0.254317221 & 0.987923327 & 0.899345598 \\
\hline 0.273128732 & 0.057319656 & 0.948644845 \\
\hline 0.228798842 & 0.133109386 & 0.935421488 \\
\hline 0.505743357 & 0.703610787 & 0.669037524 \\
\hline 0.480713307 & 0.631770357 & 0.623447371 \\
\hline 0.51482239 & 0.65197887 & 0.556353704 \\
\hline 0.4927295 & 0.736048817 & 0.534657228 \\
\hline 0.515308131 & 0.804377054 & 0.583863175 \\
\hline 0.477363193 & 0.883077568 & 0.567238961 \\
\hline
\end{tabular}




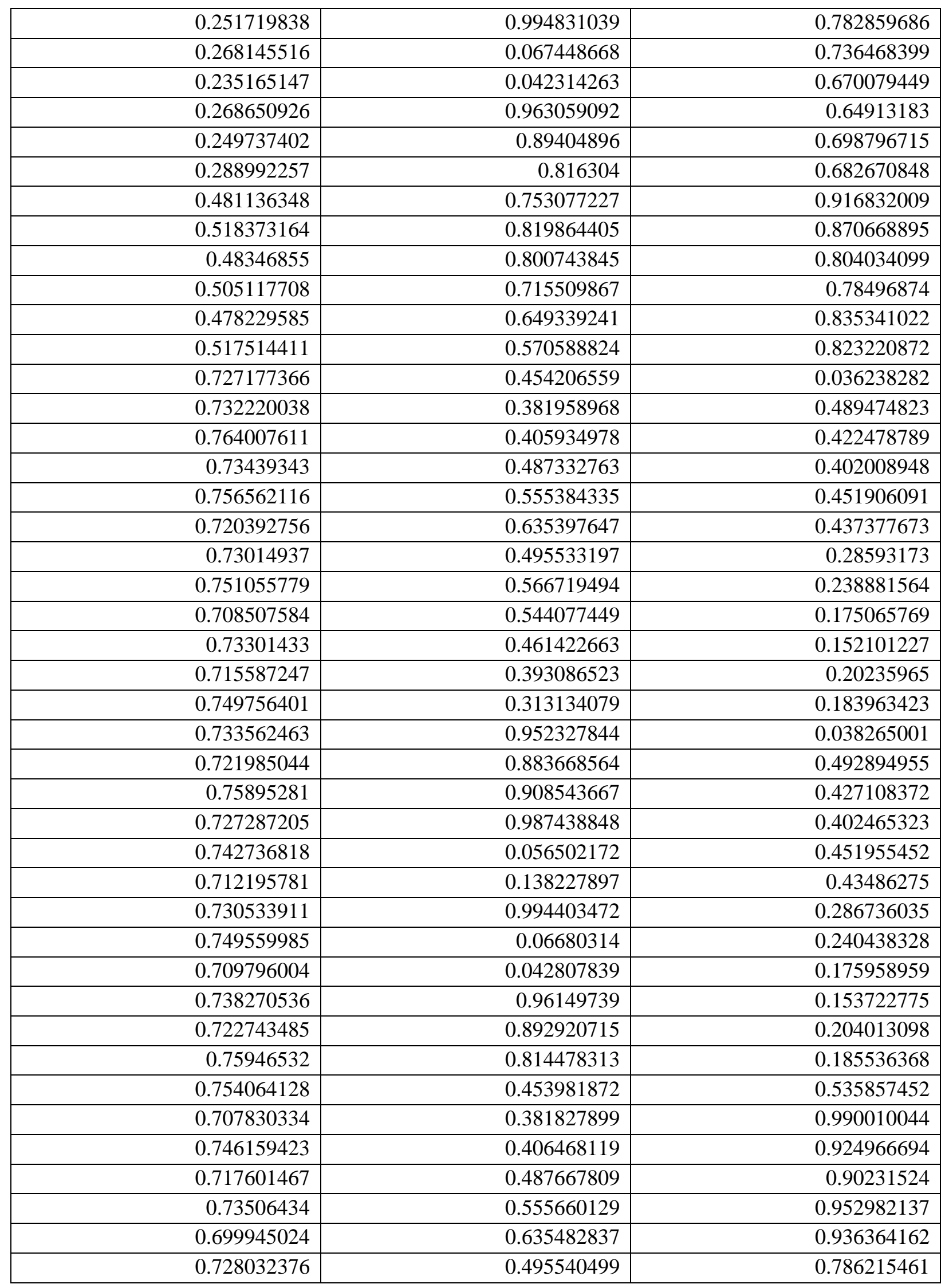




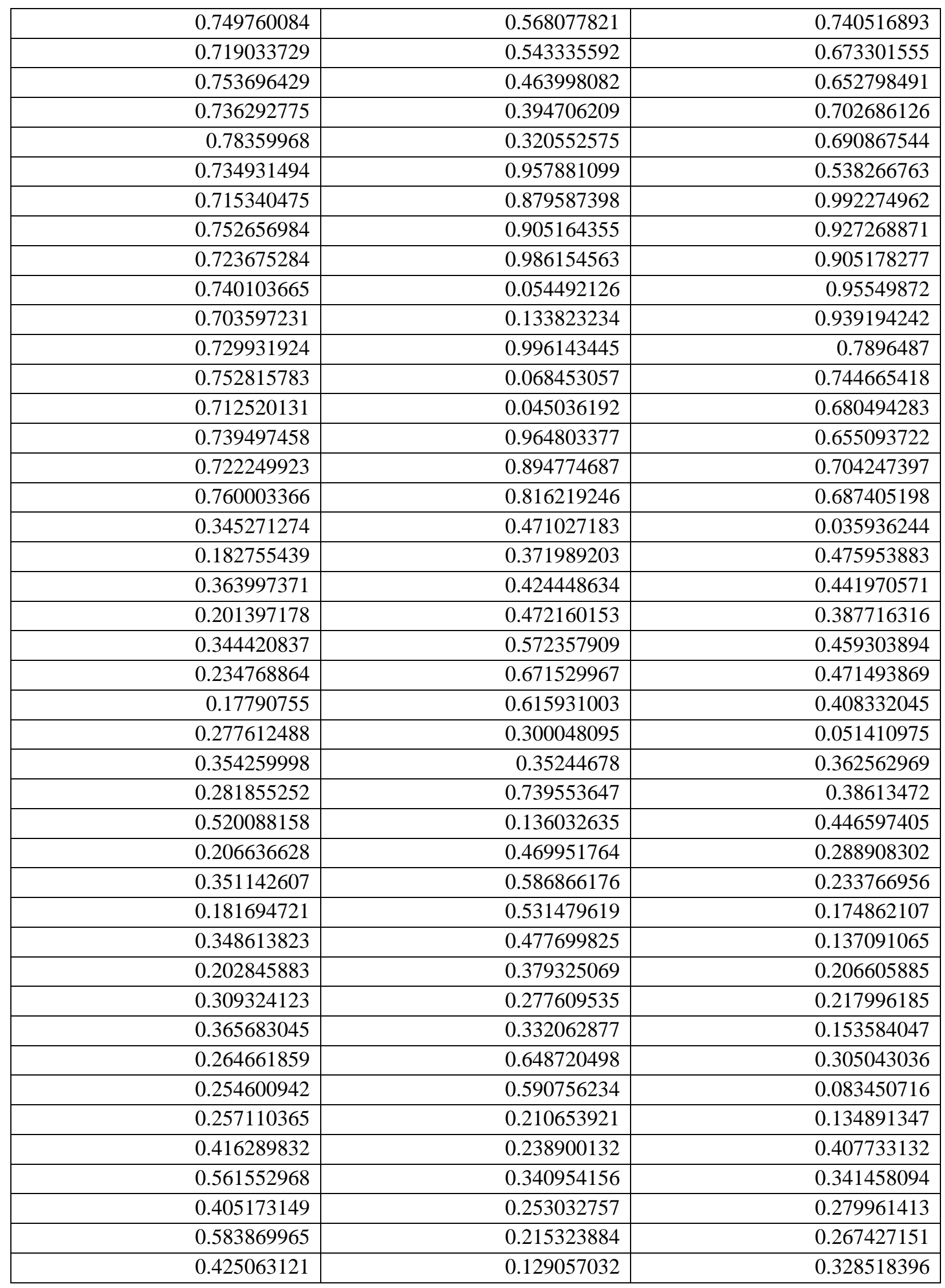




\begin{tabular}{|c|c|c|}
\hline 0.510774193 & 0.023056444 & 0.363617407 \\
\hline 0.600400019 & 0.080966245 & 0.328419871 \\
\hline 0.489418995 & 0.307692342 & 0.188048574 \\
\hline 0.509657506 & 0.963891544 & 0.268637786 \\
\hline 0.345743449 & 0.971768857 & 0.03794831 \\
\hline 0.183821975 & 0.871198238 & 0.480562222 \\
\hline 0.358290347 & 0.913784806 & 0.425789586 \\
\hline 0.195332307 & 0.978567976 & 0.389138957 \\
\hline 0.345285111 & 0.071023487 & 0.457164873 \\
\hline 0.238370609 & 0.171532961 & 0.47774687 \\
\hline 0.175147834 & 0.116934136 & 0.417696565 \\
\hline 0.281443148 & 0.802896279 & 0.05407891 \\
\hline 0.28533305 & 0.862247077 & 0.332293793 \\
\hline 0.280801725 & 0.24098451 & 0.391671662 \\
\hline 0.502380974 & 0.605943281 & 0.469148318 \\
\hline 0.20000746 & 0.985487707 & 0.28824975 \\
\hline 0.357047422 & 0.081078815 & 0.232233723 \\
\hline 0.184689591 & 0.035049646 & 0.175627893 \\
\hline 0.349764963 & 0.973786344 & 0.141050399 \\
\hline 0.196330469 & 0.881312131 & 0.204606362 \\
\hline 0.298298291 & 0.77827076 & 0.228404634 \\
\hline 0.367639166 & 0.832434941 & 0.172380217 \\
\hline 0.269146697 & 0.150985862 & 0.304597351 \\
\hline 0.257716678 & 0.089113691 & 0.082140901 \\
\hline 0.262230763 & 0.713071668 & 0.139376439 \\
\hline 0.429848496 & 0.738797409 & 0.423905183 \\
\hline 0.592899069 & 0.824289956 & 0.370486409 \\
\hline 0.420863931 & 0.794258694 & 0.309905533 \\
\hline 0.581528869 & 0.723411771 & 0.277519769 \\
\hline 0.422185203 & 0.635706644 & 0.334919139 \\
\hline 0.517729604 & 0.525436438 & 0.350699549 \\
\hline 0.595272026 & 0.589478352 & 0.307358952 \\
\hline 0.506681034 & 0.898537667 & 0.445035151 \\
\hline 0.505075027 & 0.838797056 & 0.218858303 \\
\hline 0.328892927 & 0.469522536 & 0.545390981 \\
\hline 0.190414705 & 0.366191828 & 0.986771422 \\
\hline 0.351608928 & 0.415758348 & 0.913852088 \\
\hline 0.183656462 & 0.478727067 & 0.894236421 \\
\hline 0.343225495 & 0.571031635 & 0.948721448 \\
\hline 0.240116442 & 0.673623162 & 0.9773967 \\
\hline 0.171604378 & 0.620715528 & 0.919636615 \\
\hline 0.250694286 & 0.301280102 & 0.558429832 \\
\hline 0.259116149 & 0.360597135 & 0.83001761 \\
\hline
\end{tabular}




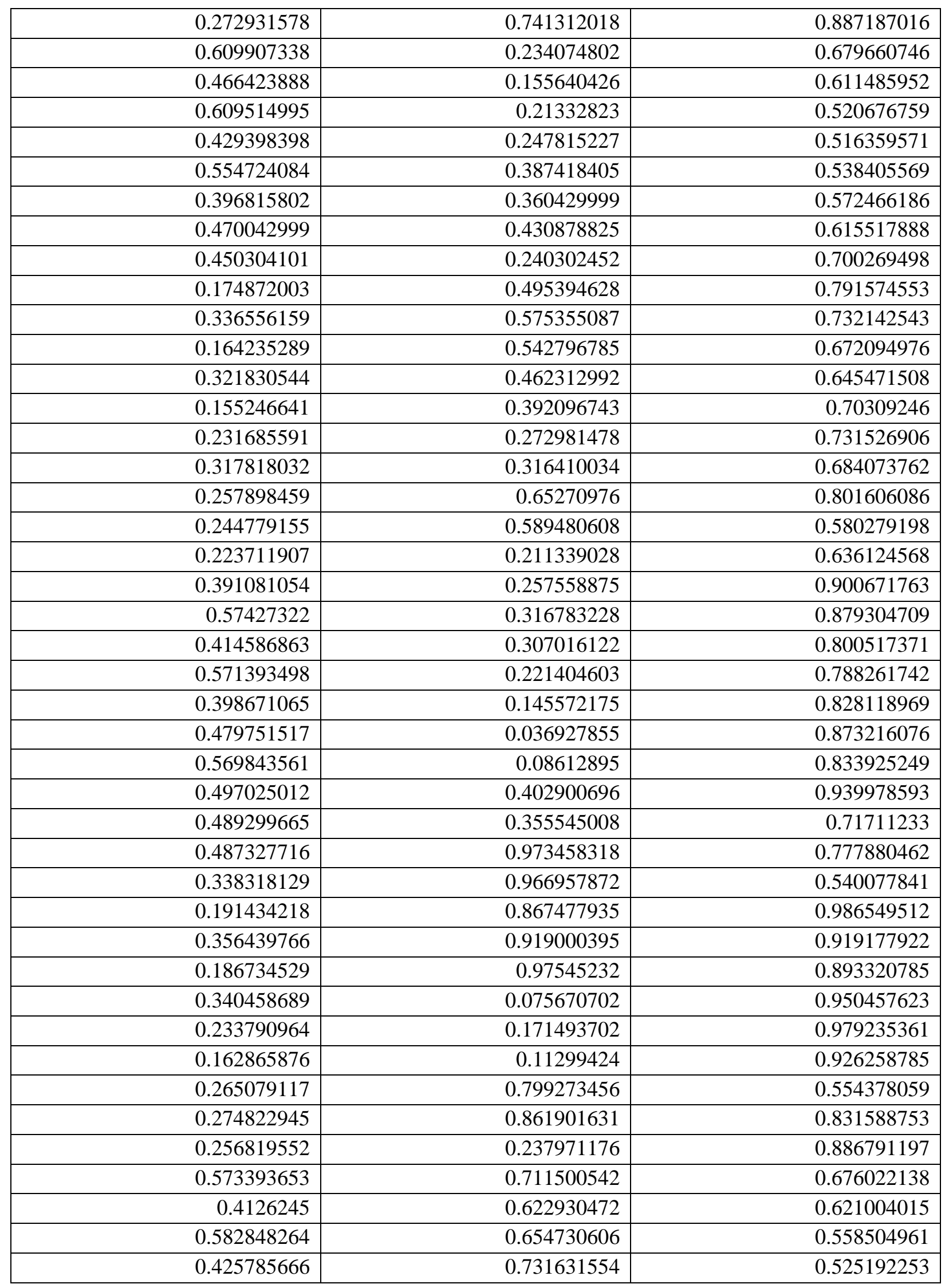




\begin{tabular}{|c|c|c|}
\hline 0.582962912 & 0.816863615 & 0.587989319 \\
\hline 0.478091358 & 0.9196075 & 0.612575019 \\
\hline 0.411934742 & 0.864756911 & 0.554934728 \\
\hline 0.496539382 & 0.552208639 & 0.693323969 \\
\hline 0.184260495 & 0.980384123 & 0.790332395 \\
\hline 0.335597764 & 0.084612546 & 0.734008179 \\
\hline 0.167153552 & 0.030104367 & 0.672978265 \\
\hline 0.336125743 & 0.974692298 & 0.642634689 \\
\hline 0.182114158 & 0.878940616 & 0.702889008 \\
\hline 0.288192866 & 0.777929507 & 0.726318712 \\
\hline 0.353684715 & 0.83325636 & 0.668341543 \\
\hline 0.244129253 & 0.146986713 & 0.803658008 \\
\hline 0.243831827 & 0.087884684 & 0.581936666 \\
\hline 0.250734041 & 0.711674385 & 0.637960056 \\
\hline 0.413166678 & 0.749716578 & 0.916577639 \\
\hline 0.585863719 & 0.8187376 & 0.869962852 \\
\hline 0.415619173 & 0.799374401 & 0.805717289 \\
\hline 0.572501589 & 0.718804308 & 0.778068899 \\
\hline 0.410404672 & 0.636523472 & 0.834620667 \\
\hline 0.512339465 & 0.534284252 & 0.867794307 \\
\hline 0.583446014 & 0.587347644 & 0.812007456 \\
\hline 0.512249353 & 0.901996146 & 0.93696113 \\
\hline 0.499688237 & 0.844166017 & 0.715966241 \\
\hline 0.49231092 & 0.466001172 & 0.774826682 \\
\hline 0.794999798 & 0.466737518 & 0.042235782 \\
\hline 0.664208978 & 0.370324975 & 0.487025298 \\
\hline 0.832194437 & 0.41401281 & 0.423576615 \\
\hline 0.666680334 & 0.478718876 & 0.396042325 \\
\hline 0.824432167 & 0.568050589 & 0.45561087 \\
\hline 0.723548007 & 0.672672227 & 0.481751311 \\
\hline 0.655018476 & 0.621771911 & 0.423488296 \\
\hline 0.720401346 & 0.299046242 & 0.057651494 \\
\hline 0.749632992 & 0.360366315 & 0.334124566 \\
\hline 0.758815589 & 0.739055487 & 0.39261535 \\
\hline 0.66274405 & 0.485369026 & 0.294307818 \\
\hline 0.818665323 & 0.573931816 & 0.231481769 \\
\hline 0.640982211 & 0.538764171 & 0.1826007 \\
\hline 0.799933826 & 0.468904849 & 0.14204288 \\
\hline 0.648380286 & 0.380724187 & 0.210640728 \\
\hline 0.753955209 & 0.27617584 & 0.2279855 \\
\hline 0.812618078 & 0.328048062 & 0.164160628 \\
\hline 0.74474279 & 0.649756977 & 0.308177268 \\
\hline 0.712271205 & 0.58898756 & 0.086158272 \\
\hline
\end{tabular}




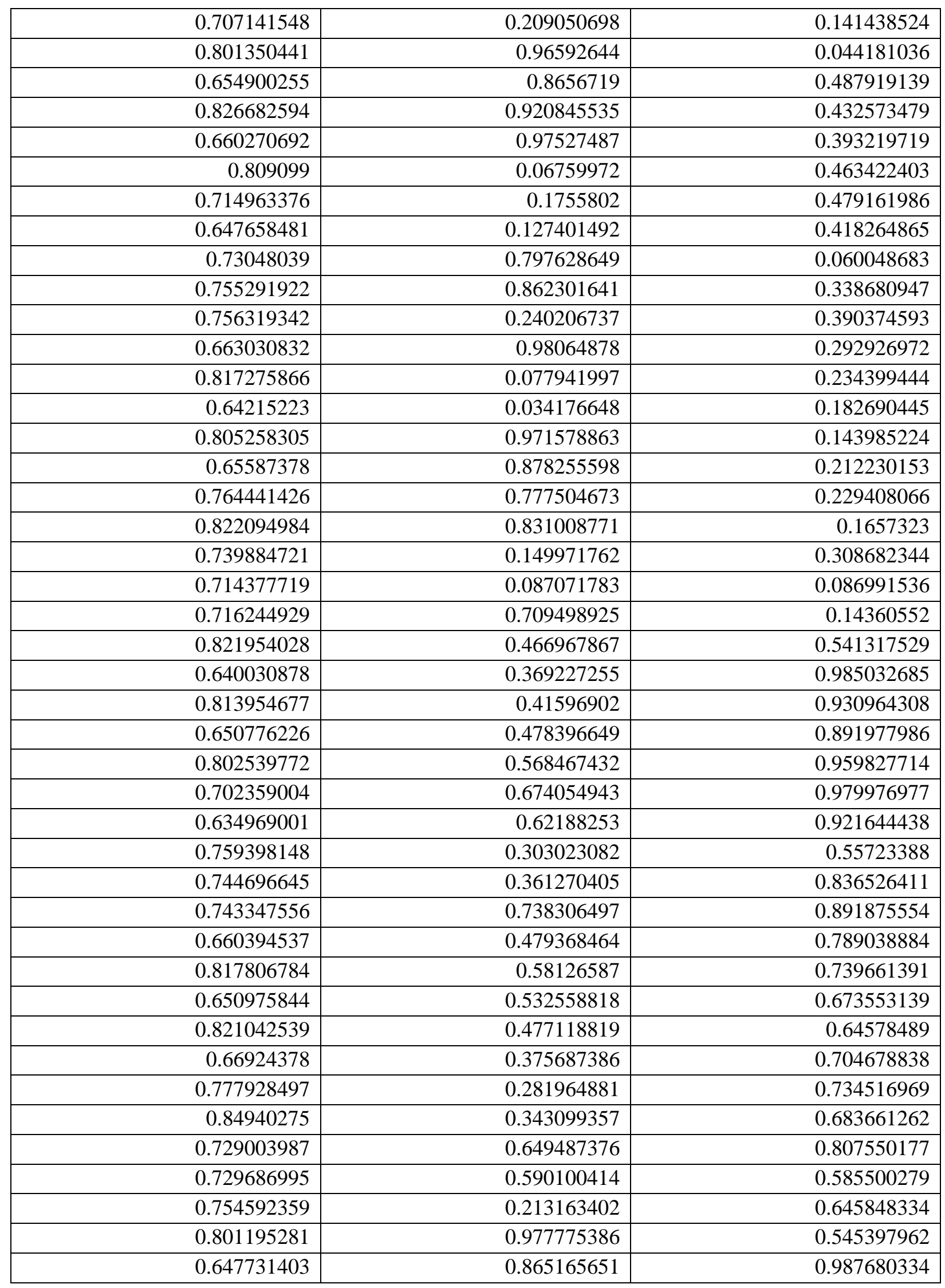




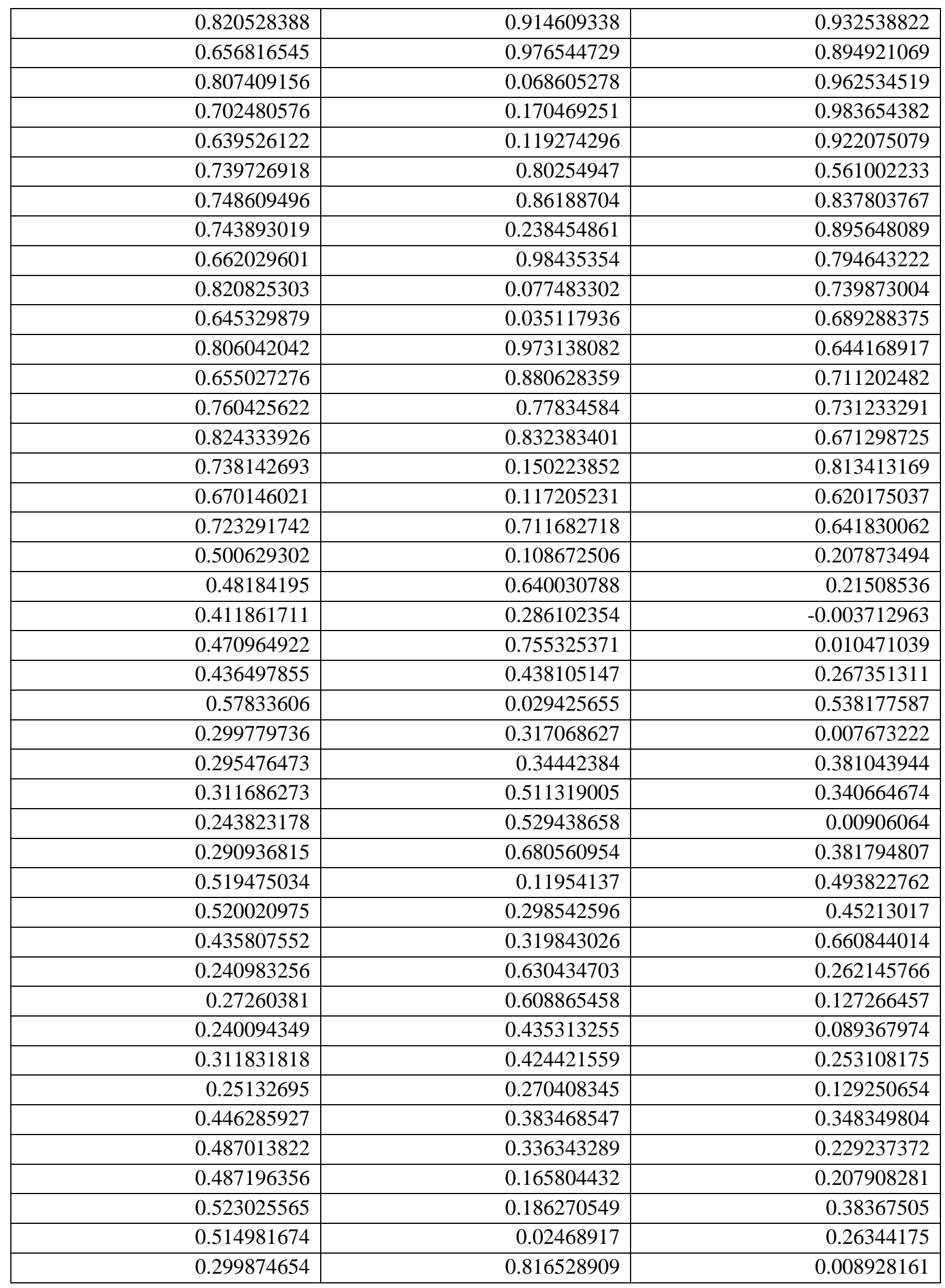




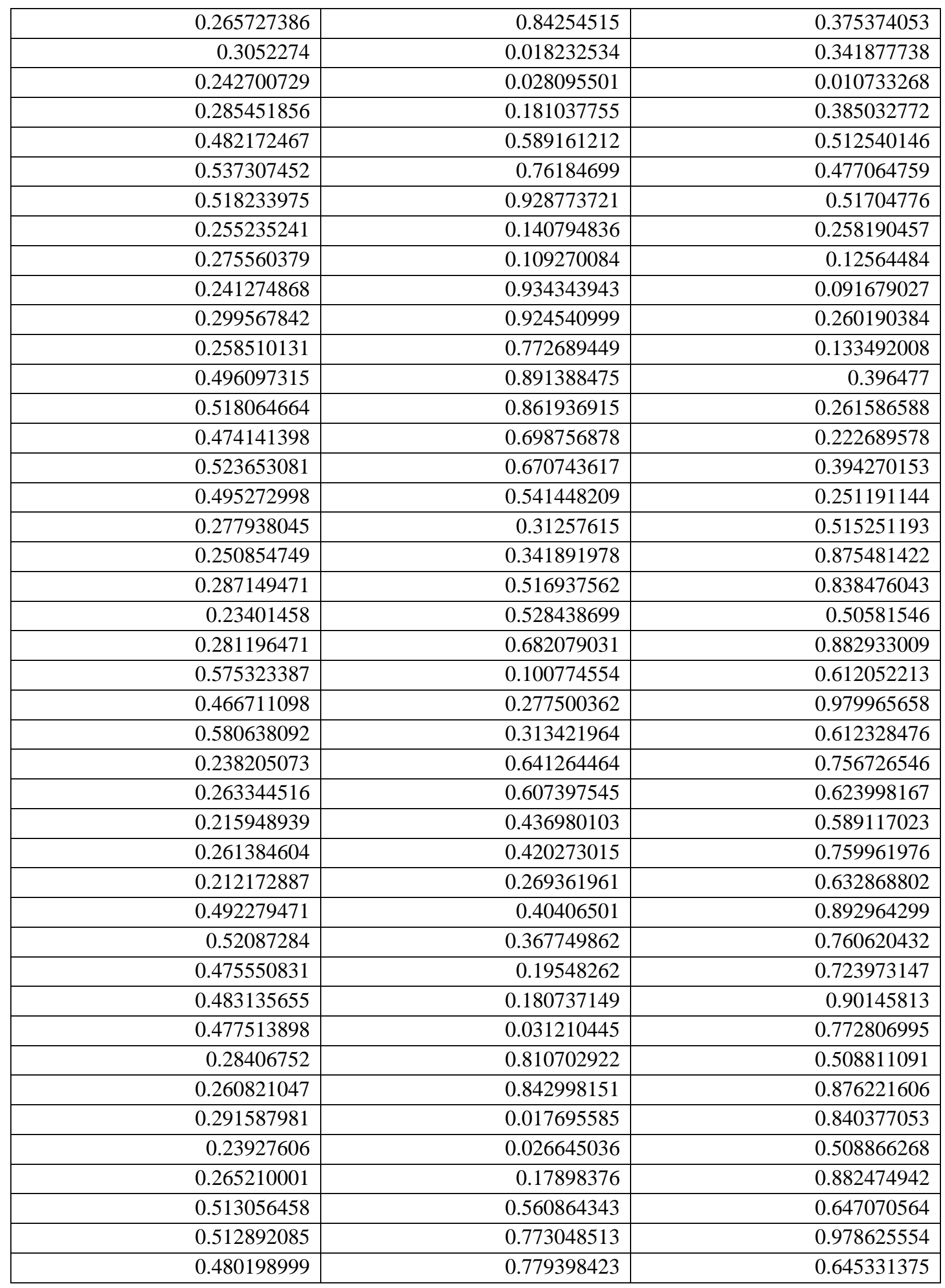




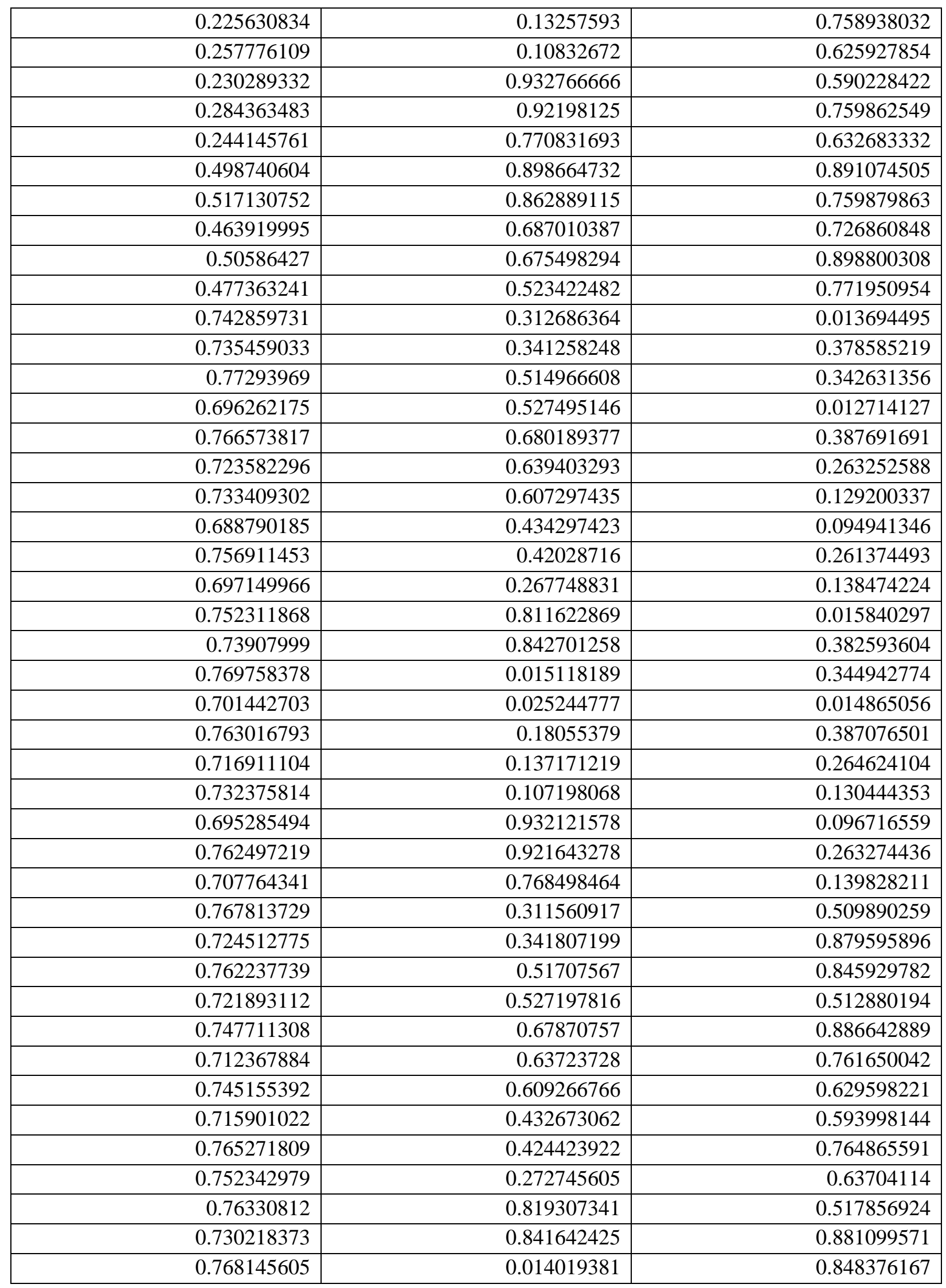




\begin{tabular}{|r|r|r|}
\hline 0.698674727 & 0.027292114 & 0.509614551 \\
\hline 0.753330273 & 0.179796772 & 0.892118531 \\
\hline 0.721214781 & 0.139966394 & 0.767370941 \\
\hline 0.72866816 & 0.108934066 & 0.634198349 \\
\hline 0.694485596 & 0.940474748 & 0.596393997 \\
\hline 0.76047132 & 0.922500352 & 0.764495855 \\
\hline 0.712826652 & 0.770404728 & 0.638592611 \\
\hline
\end{tabular}

Mg-Int-Mg co-ordinates:

$\mathrm{a}=16.352 \AA, \mathrm{b}=16.402 \AA, \mathrm{c}=20.760 \AA$

$\Upsilon=96.550$

C $\quad \mathrm{H} \quad \mathrm{Mg} \quad \mathrm{O}$

$\begin{array}{llll}132 & 220 & 2 & 112\end{array}$

\begin{tabular}{|c|c|c|}
\hline $\mathrm{x}$ & $\mathrm{y}$ & $\mathrm{z}$ \\
\hline 0.273167369 & 0.458413517 & 0.031682755 \\
\hline 0.261549126 & 0.383258145 & 0.488041033 \\
\hline 0.307228064 & 0.409626126 & 0.426389949 \\
\hline 0.272244656 & 0.485512362 & 0.399371486 \\
\hline 0.281733677 & 0.556404595 & 0.448358848 \\
\hline 0.240939929 & 0.632252709 & 0.428888371 \\
\hline 0.274270133 & 0.494375688 & 0.28295655 \\
\hline 0.285374189 & 0.568967749 & 0.237894859 \\
\hline 0.248949945 & 0.544561948 & 0.172017592 \\
\hline 0.279116891 & 0.46521723 & 0.147225308 \\
\hline 0.266533789 & 0.395566932 & 0.196069461 \\
\hline 0.299195046 & 0.315647709 & 0.175536917 \\
\hline 0.483770498 & 0.256913886 & 0.390495816 \\
\hline 0.497537816 & 0.31635298 & 0.334249118 \\
\hline 0.473966738 & 0.27203851 & 0.27151652 \\
\hline 0.517304052 & 0.197084101 & 0.260366715 \\
\hline 0.492261362 & 0.140812961 & 0.318313611 \\
\hline 0.531316212 & 0.06114397 & 0.31752678 \\
\hline 0.2756078 & 0.95664259 & 0.03398472 \\
\hline 0.252861659 & 0.88258982 & 0.486340319 \\
\hline 0.290963605 & 0.906973521 & 0.421257744 \\
\hline 0.263837269 & 0.989370618 & 0.399878859 \\
\hline 0.281286954 & 0.056952517 & 0.450836572 \\
\hline 0.242490085 & 0.135502639 & 0.435489468 \\
\hline 0.269203769 & 0.998969358 & 0.283877491 \\
\hline 0.289722277 & 0.070525051 & 0.236965759 \\
\hline 0.251299669 & 0.0449933 & 0.172200552 \\
\hline
\end{tabular}

Facas et al. 


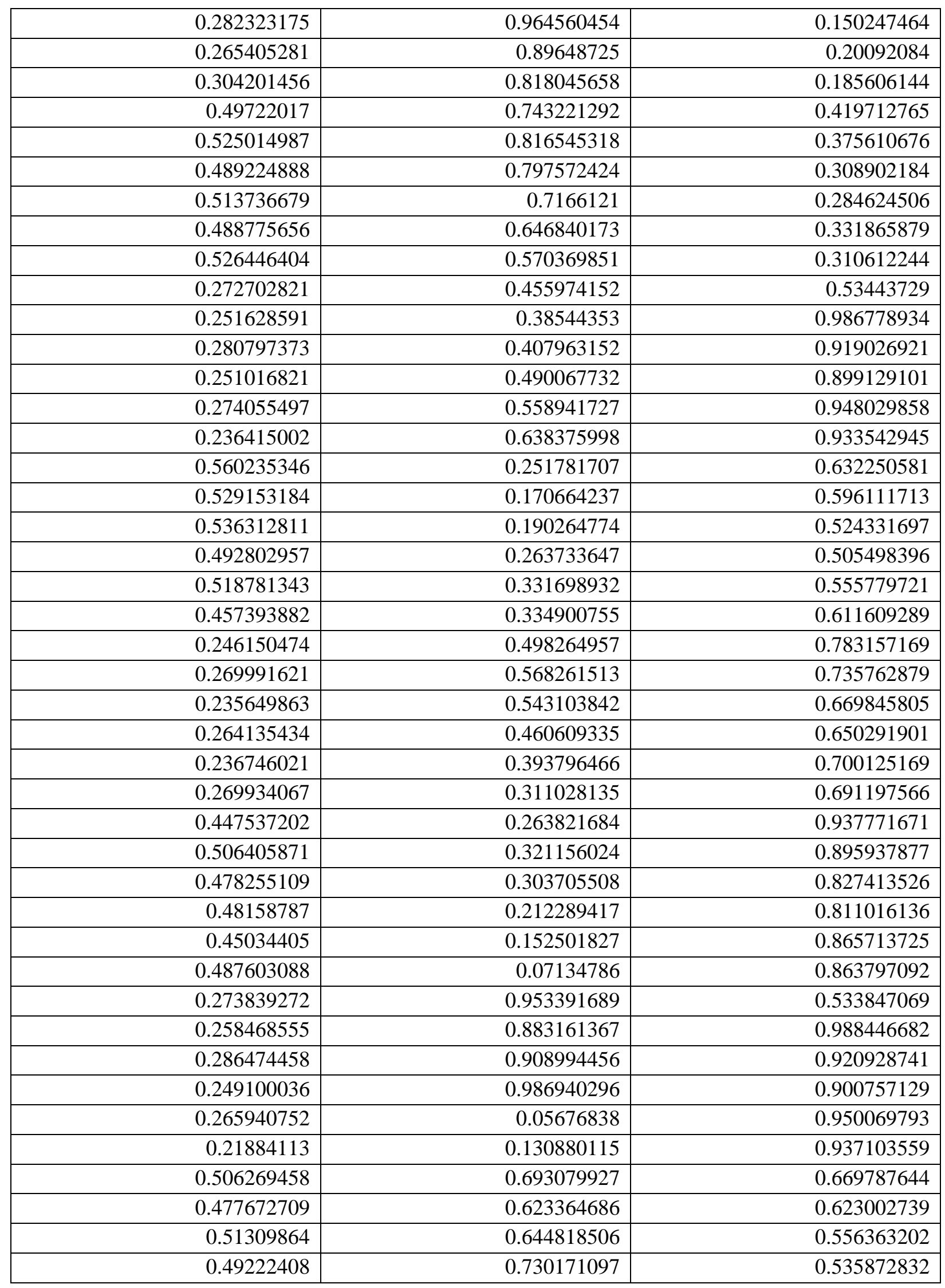




\begin{tabular}{|c|c|c|}
\hline 0.51607266 & 0.796807889 & 0.586173305 \\
\hline 0.47847042 & 0.876845508 & 0.572889191 \\
\hline 0.246994282 & 0.993649704 & 0.783818826 \\
\hline 0.263583673 & 0.066589768 & 0.737847859 \\
\hline 0.235672441 & 0.041774383 & 0.670255484 \\
\hline 0.269754336 & 0.962536803 & 0.650181342 \\
\hline 0.249842806 & 0.893613807 & 0.699678098 \\
\hline 0.28936525 & 0.815826029 & 0.684010556 \\
\hline 0.480002772 & 0.746658427 & 0.917281371 \\
\hline 0.518925613 & 0.812047029 & 0.870755184 \\
\hline 0.485725566 & 0.792122507 & 0.803671835 \\
\hline 0.506595079 & 0.706063212 & 0.785853888 \\
\hline 0.477886672 & 0.641266962 & 0.836858195 \\
\hline 0.517382447 & 0.562232777 & 0.826922464 \\
\hline 0.730025348 & 0.456605205 & 0.038401877 \\
\hline 0.735263047 & 0.385192941 & 0.491973958 \\
\hline 0.766335702 & 0.408456602 & 0.424694237 \\
\hline 0.73653872 & 0.489684351 & 0.404085356 \\
\hline 0.75917541 & 0.558329279 & 0.453415224 \\
\hline 0.722182697 & 0.637797802 & 0.438690601 \\
\hline 0.731671787 & 0.497445565 & 0.287973725 \\
\hline 0.75157418 & 0.568808535 & 0.240818182 \\
\hline 0.709254815 & 0.545623526 & 0.177034207 \\
\hline 0.734856985 & 0.463351206 & 0.154206434 \\
\hline 0.717708683 & 0.39498583 & 0.204468185 \\
\hline 0.752280811 & 0.315130346 & 0.18639685 \\
\hline 0.73894515 & 0.954634361 & 0.038913341 \\
\hline 0.719012123 & 0.884765541 & 0.494163549 \\
\hline 0.756486651 & 0.909637812 & 0.428542476 \\
\hline 0.724721589 & 0.988229337 & 0.403341934 \\
\hline 0.738983735 & 0.057408024 & 0.452862275 \\
\hline 0.709269386 & 0.139353005 & 0.435145298 \\
\hline 0.730092096 & 0.995343878 & 0.287560538 \\
\hline 0.750103608 & 0.068215362 & 0.241854539 \\
\hline 0.71212542 & 0.0450972 & 0.176649281 \\
\hline 0.740647393 & 0.963854653 & 0.154448605 \\
\hline 0.723541554 & 0.894879766 & 0.204228703 \\
\hline 0.759458759 & 0.816000232 & 0.185721737 \\
\hline 0.758545676 & 0.457592327 & 0.537866764 \\
\hline 0.712838466 & 0.383784056 & 0.99236874 \\
\hline 0.750748547 & 0.408668768 & 0.927257798 \\
\hline 0.720716735 & 0.489142899 & 0.904398816 \\
\hline 0.736262142 & 0.557447308 & 0.954985747 \\
\hline
\end{tabular}




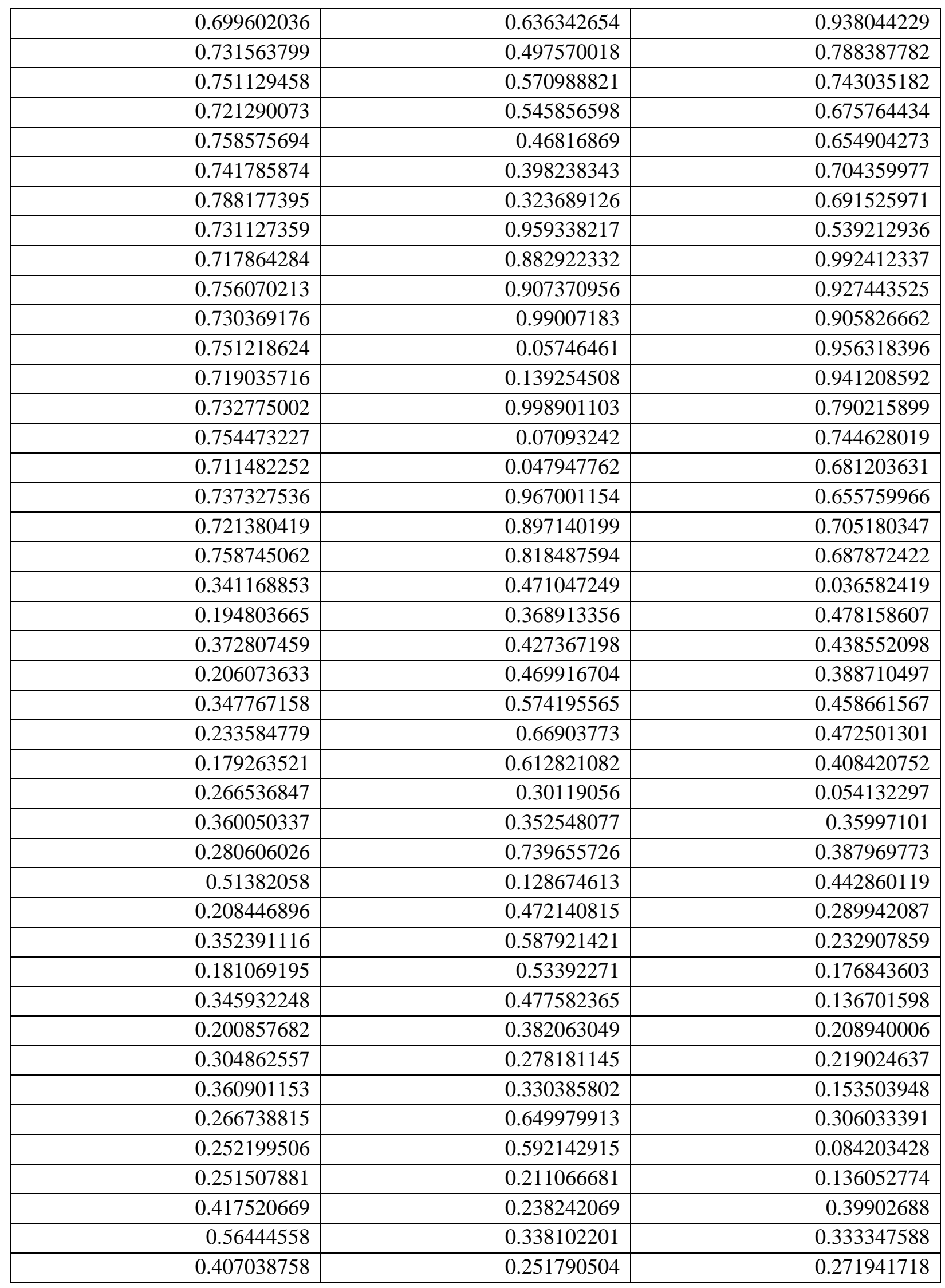




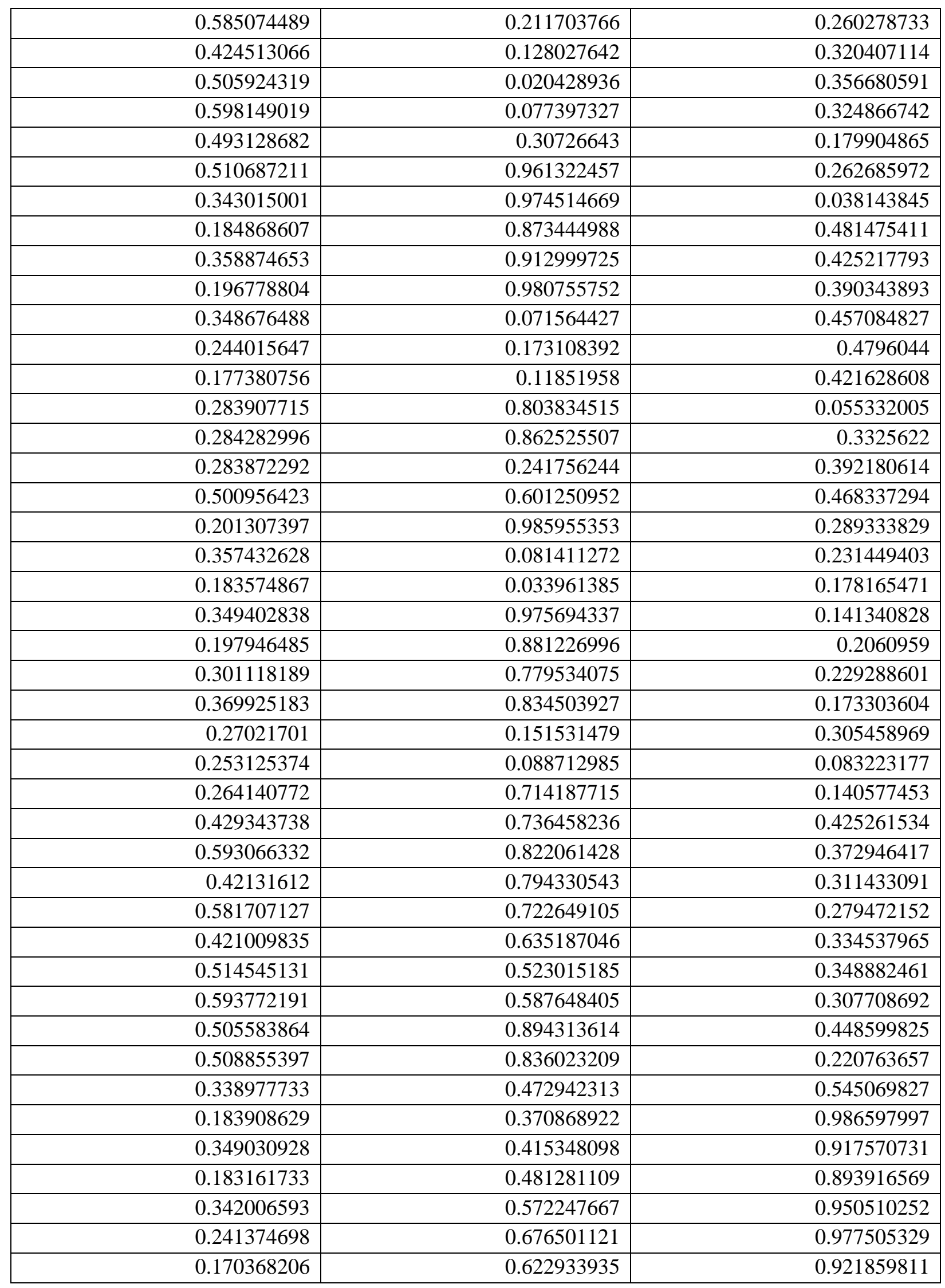




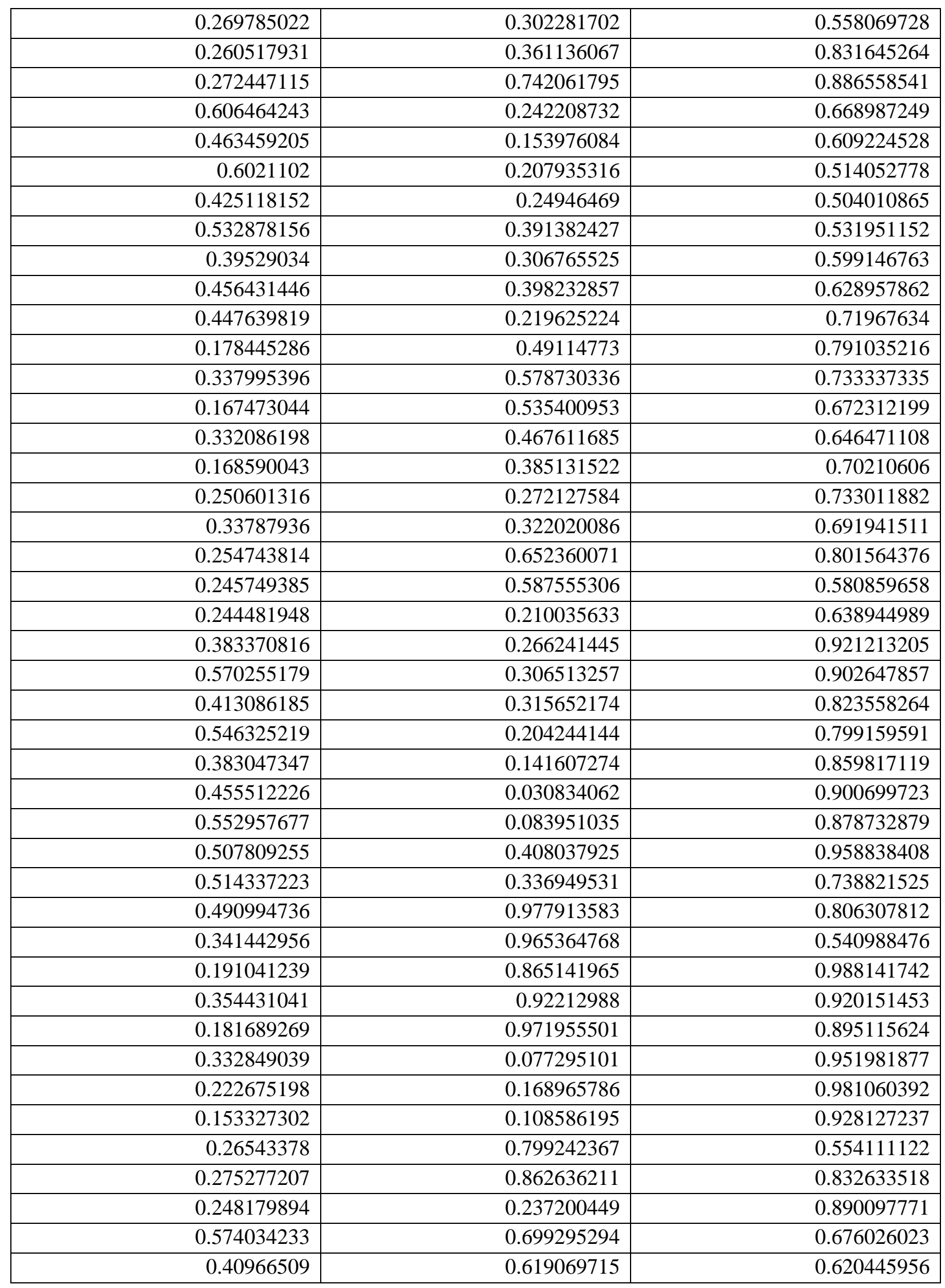




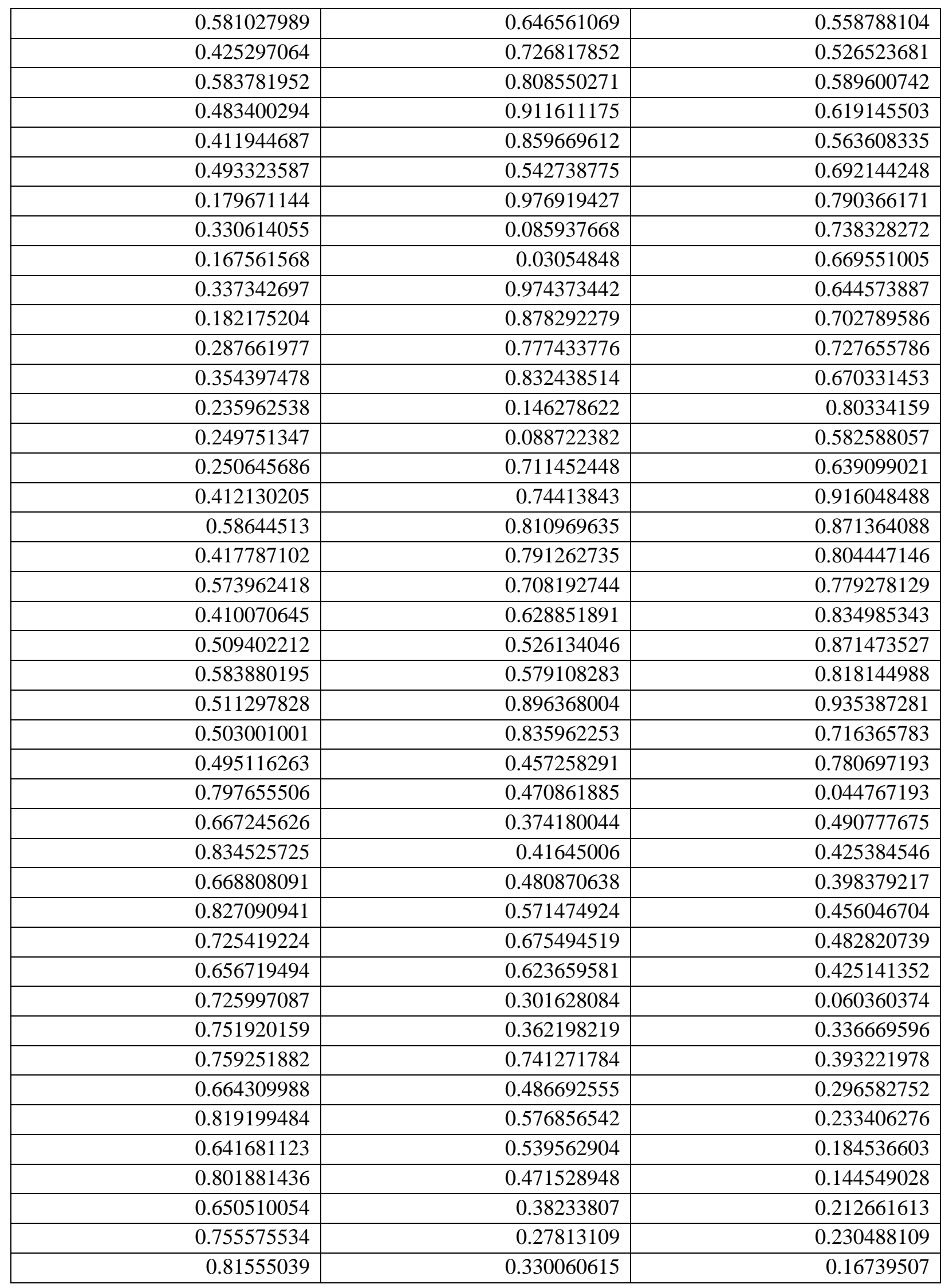




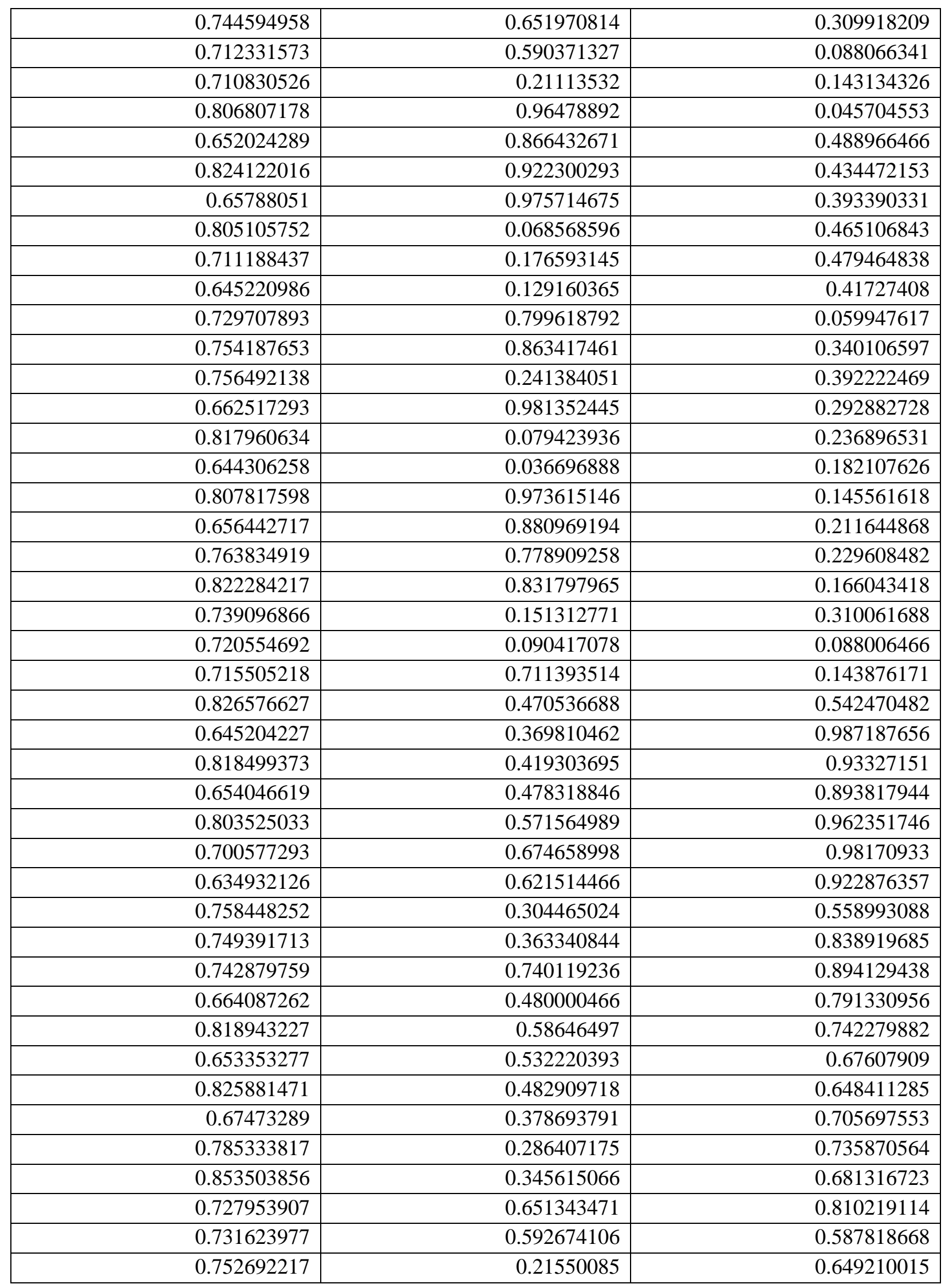




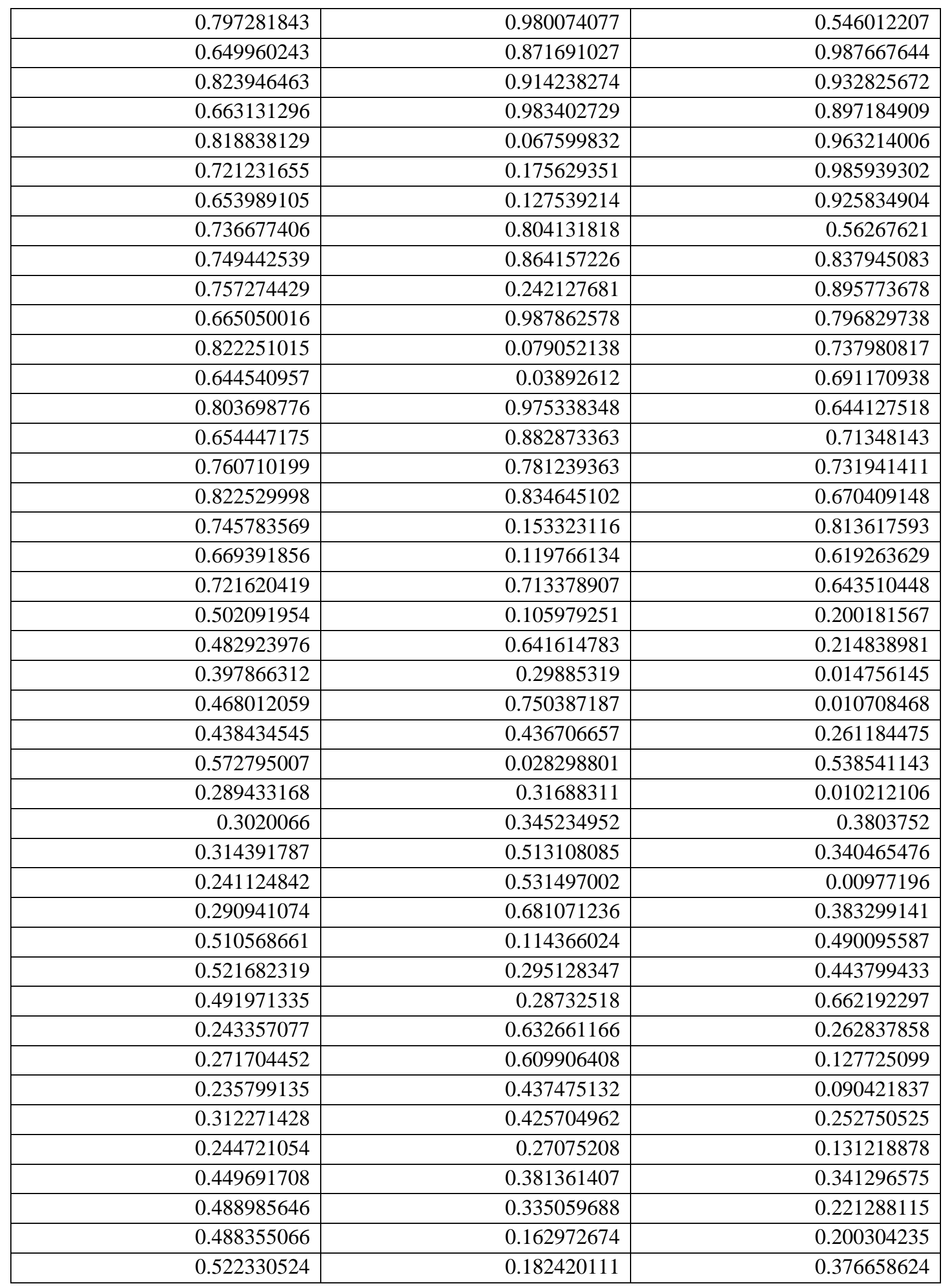




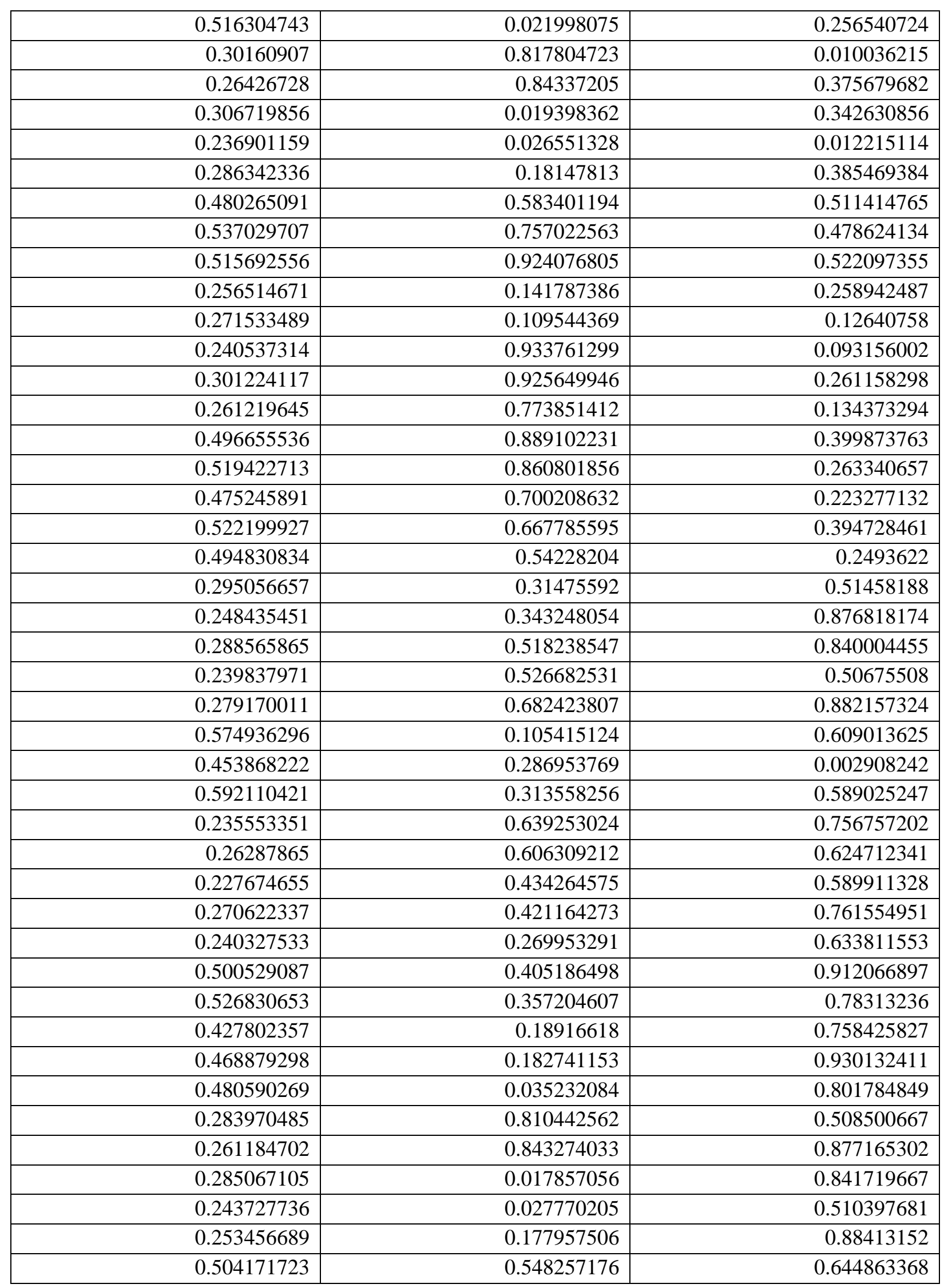




\begin{tabular}{|c|c|c|}
\hline 0.51076126 & 0.767203887 & 0.97925349 \\
\hline 0.481828547 & 0.769891785 & 0.647507825 \\
\hline 0.218191128 & 0.130611501 & 0.758764207 \\
\hline 0.263006284 & 0.107832716 & 0.627099575 \\
\hline 0.232640049 & 0.932418129 & 0.590793983 \\
\hline 0.282727626 & 0.922128264 & 0.761133239 \\
\hline 0.245066515 & 0.770691453 & 0.633530267 \\
\hline 0.498830137 & 0.891491704 & 0.889410648 \\
\hline 0.52098802 & 0.853962457 & 0.760248169 \\
\hline 0.465319932 & 0.676144244 & 0.727935724 \\
\hline 0.503891001 & 0.668201285 & 0.900529966 \\
\hline 0.480889926 & 0.514138845 & 0.774393118 \\
\hline 0.749263026 & 0.315887141 & 0.016695345 \\
\hline 0.737434105 & 0.343514957 & 0.381101229 \\
\hline 0.7746737 & 0.517233367 & 0.34447573 \\
\hline 0.697293812 & 0.528700632 & 0.014494484 \\
\hline 0.767733332 & 0.682524454 & 0.388578116 \\
\hline 0.723079494 & 0.641055319 & 0.265094949 \\
\hline 0.733354869 & 0.609009375 & 0.131048885 \\
\hline 0.691366273 & 0.435919534 & 0.096835804 \\
\hline 0.758980717 & 0.422492579 & 0.263345061 \\
\hline 0.700567446 & 0.269802399 & 0.140265376 \\
\hline 0.751392338 & 0.812761846 & 0.015564445 \\
\hline 0.737704527 & 0.843663233 & 0.383905299 \\
\hline 0.768241829 & 0.015684223 & 0.346220952 \\
\hline 0.711200829 & 0.029670624 & 0.01556431 \\
\hline 0.761878889 & 0.181517891 & 0.388230061 \\
\hline 0.716790158 & 0.138299375 & 0.265791229 \\
\hline 0.73652553 & 0.109984994 & 0.131995085 \\
\hline 0.698666872 & 0.935511505 & 0.096720725 \\
\hline 0.762441833 & 0.922698834 & 0.26405307 \\
\hline 0.707476517 & 0.770487873 & 0.139887587 \\
\hline 0.770745555 & 0.314854958 & 0.512353133 \\
\hline 0.730014879 & 0.343553943 & 0.882072466 \\
\hline 0.765328235 & 0.519336783 & 0.84820381 \\
\hline 0.726172588 & 0.530968582 & 0.515017336 \\
\hline 0.747233912 & 0.680564566 & 0.888714438 \\
\hline 0.711016904 & 0.638275252 & 0.764460517 \\
\hline 0.744675541 & 0.612552484 & 0.632194249 \\
\hline 0.721350342 & 0.436950726 & 0.596288428 \\
\hline 0.770160336 & 0.427517815 & 0.766758579 \\
\hline 0.752577009 & 0.275157504 & 0.639805935 \\
\hline 0.760578217 & 0.820851205 & 0.519618483 \\
\hline
\end{tabular}




\begin{tabular}{|r|r|r|}
\hline 0.731237519 & 0.844428871 & 0.881425811 \\
\hline 0.773468601 & 0.016446606 & 0.848166578 \\
\hline 0.693935051 & 0.02787677 & 0.51003824 \\
\hline 0.768246374 & 0.183764494 & 0.893122444 \\
\hline 0.725251191 & 0.143029802 & 0.768580561 \\
\hline 0.727514802 & 0.111328311 & 0.634633803 \\
\hline 0.691406629 & 0.942026478 & 0.597625345 \\
\hline 0.761605563 & 0.924850515 & 0.764668332 \\
\hline 0.710093098 & 0.771832031 & 0.640330205 \\
\hline
\end{tabular}

